Princípio da similaridade para classes de campos vetoriais complexos 

SERVIÇO DE PÓS-GRADUAÇÃO DO ICMC-USP

Data de Depósito:

Assinatura:

\title{
Princípio da similaridade para classes de campos vetoriais complexos
}

\author{
Sabrina Graciela Suárez Calcina
}

Orientador: Prof. Dr. Paulo Leandro Dattori da Silva

Dissertação apresentada ao Instituto de Ciências Matemáticas e de Computação - ICMC-USP, como parte dos requisitos para obtenção do título de Mestre em Ciências - Matemática . VERSÃO REVISADA 
Ficha catalográfica elaborada pela Biblioteca Prof. Achille Bassi e Seção Técnica de Informática, ICMC/USP, com os dados fornecidos pelo(a) autor(a)

C144p Calcina, Sabrina Graciela Suárez
Princípio da similaridade para classes de campos
vetoriais complexos / Sabrina Graciela Suárez
Calcina; orientador Paulo Leandro Dattori da
Silva. -- São Carlos, 2014 .
110 p.
Dissertação (Mestrado - Programa de Pós-Graduação
ematemática) -- Instituto de Ciências Matemáticas
e de Computação, Universidade de São Paulo, 2014.
1. Princípio da similaridade. 2. Campos
vetoriais. 3. Funços pseudo-analíticas. 4. Funções
analiticas. I. Dattori da Silva, Paulo Leandro,
orient. II. Título.


"A Matemática é o mais maravilhoso instrumento criado pelo gênio do homem para a descoberta da Verdade." Laisant 



\section{Agradecimentos}

À Deus, minha imensa gratidão por dar-me forças a cada dia para não desistir e pelo amor que me faz acreditar num futuro melhor.

Ao programa de pós graduação do Instituto de Ciências Matemáticas e de Computação.

Aos professores, em especial ao meu orientador e amigo, Paulo Leandro Dattori da Silva, pela disponibilidade, grande dedicação e pelos conhecimentos transmitidos.

À minha família, em especial para minha mãe Julia, meus tios: Gregório, Isidro, Francisco, Mario e Leonidas, e meus irmãos; pela força, coragem e apoio incondicional para eu seguir em frente. Amo demais todos vocês.

Aos meus amigos e colegas, Camilo Campana, Jean Cerqueira, Lito Bocanegra, Patricia Hilario e Norbil Cordova, sou grata pela ajuda que deram sempre que precisei.

À uma pessoa muito especial que faz parte da minha vida, Álvaro Yucra, pelo carinho e apoio incondicional, pela atenção, paciência e compreensão, pelas palavras de estímulo que me ajudarem a superar as dificuldades na minha vida.

À todos os citados e as pessoas que não mencionei, mas que fizeram parte da minha trajetória, fica registrado o meu agradecimento.

À CAPES pelo apoio financeiro. 


\section{Resumo}

Esta dissertação trata do Princípio da similaridade para as soluções das equações da forma

$$
L \omega=A(z) \cdot \omega+B(z) \cdot \bar{\omega}
$$

sendo $L$ um campo vetorial complexo não singular e $A, B \in C^{\sigma}\left(\mathbb{R}^{2}\right)$, com $0<\sigma<1$.

Aqui são apresentados resultados para o campo vetorial elítico $L=\frac{\partial}{\partial \bar{z}}$ e para classes de campos vetoriais elíticos degenerados. 


\section{Abstract}

This dissertation deals with the Similarity principle for solutions of equations of the form

$$
L \omega=A(z) \cdot \omega+B(z) \cdot \bar{\omega}
$$

where $L$ is a nonsingular complex vector field and $A, B \in C^{\sigma}\left(\mathbb{R}^{2}\right)$, with $0<\sigma<1$.

Here are presented results for elliptic vector field and for classes of degenerate elliptic vector fields. 


\section{Sumário}

Introdução

1 Preliminares 3

1.1 Alguns resultados de Análise Real . . . . . . . . . . . . . . . . . . . 3

1.2 Alguns resultados de Análise Complexa . . . . . . . . . . . . . . . . . . . . 10

1.3 Elementos básicos sobre distribuições . . . . . . . . . . . . . . . . . . . . . 11

1.3.1 Alguns fatos sobre convoluções . . . . . . . . . . . . . . . . . 13

1.4 Sistemas não homogêneos de Cauchy-Riemann . . . . . . . . . . . . . . . . 16

1.5 Fórmula integral de Cauchy não homogênea . . . . . . . . . . . . . . 17

1.6 Funções Hölder contínuas . . . . . . . . . . . . . . . . . . . 20

1.6.1 Lemas sobre funções Hölder contínuas . . . . . . . . . . . . . . . . 21

1.7 Operador integral . . . . . . . . . . . . . . . . . . . 30

1.8 Mudança de variáveis em campos vetoriais . . . . . . . . . . . . . 36

2 Princípio da similaridade para o campo vetorial $\frac{\partial}{\partial \bar{z}} \quad 39$

2.1 Função $[\mathrm{a}, \mathrm{b}]-$ pseudo-analítica . . . . . . . . . . . . . . . . . . . . . . . . . . 39

2.1.1 Equação integral . . . . . . . . . . . . . . . . . . . . . 40

2.2 Princípio da similaridade clássico f . . . . . . . . . . . . . . . 57

3 Princípio da similaridade para campos vetoriais complexos não elíticos 71

3.1 Princípio da similaridade para alguns campos vetoriais complexos de tipo finito . . . . . . . . . . . . . . . . . . . . . 72

3.2 Princípio da similaridade para um campo vetorial complexo de tipo infinito 92 


\section{Introdução}

Na década de 1950, as primeiras representações sobre as funções pseudo-analíticas aparecem nos livros de Lipman Bers "Teoria das Funções Pseudo-analíticas"(1953) e Ilia N. Vekua "Funções Analíticas Generalizadas"(1959). Em termos breves, os trabalhos eram distintos, pois Bers construiu sua teoria generalizando praticamente cada um dos principais teoremas da Teoria das Funções Analíticas, enquanto Vekua desenvolveu seu estudo a partir da perspectiva da Teoria dos Operadores Diferenciais.

Com os métodos da teoria das funções de variável complexa podemos encontrar as soluções da equação

$$
\frac{\partial \omega}{\partial \bar{z}}+a(z) \cdot \omega+b(z) \cdot \bar{\omega}=g, \quad[* 1]
$$

sendo $a, b, g$ funções suaves. Quando $g \equiv 0$ as funções que satisfazem a equação [*1] são chamadas Funções Analíticas Generalizadas (no sentido de Vekua).

R. Courant e L. Hilbert apresentaram o Princípio da Similaridade para a equação

$$
\frac{\partial \omega}{\partial \bar{z}}=a(z) \cdot \omega+b(z) \cdot \bar{\omega}
$$

sendo $a, b$ coeficientes Hölder contínuos. O Princípio da Similaridade diz que cada solução $\omega \in C^{1}$ de $[* 2]$ tem a forma

$$
\omega(x, y)=e^{s(x, y)} \cdot f(x, y), \quad[* 3]
$$

sendo $f$ uma função holomorfa e $s$ uma função Hölder contínua. Reciprocamente, para cada função holomorfa $f$, existe uma função $s$, Hölder contínua, que se anula em um ponto fixado $z_{0}$, tal que $\omega(x, y)=e^{s(x, y)} \cdot f(x, y)$ satisfaz [*2].

Sabemos que o Princípio da Similaridade vale também para um campo vetorial elítico arbitrário L, pois qualquer campo vetorial localmente elítico é um múltiplo não singular de $\partial / \partial \bar{z}$ em coordenadas apropriadas. 
No entanto, surge a seguinte pergunta sobre este problema: se $L$ é um campo vetorial complexo não singular e não elítico definido em um aberto $U$ de $\mathbb{R}^{2}$ que contem a origem, será que é possível obter o Princípio da Similaridade para esses campos vetoriais ?

No capítulo 3, esta pergunta é respondida para os Campos vetoriais complexos não singulares e não elíticos

$$
\begin{aligned}
L_{k} & =\frac{\partial}{\partial y}-i(2 k+1) y^{2 k} \frac{\partial}{\partial x}, k \in \mathbb{Z}^{+} \\
L & =\frac{\partial}{\partial y}-i x \frac{\partial}{\partial x} .
\end{aligned}
$$

Veremos que, se $\omega \in C^{1}$ é solução da equação

$$
L_{k} \omega=A \cdot \omega+B \cdot \bar{\omega}
$$

em um aberto conexo limitado $U$ que contem a origem, sendo $A, B \in C^{2 k+\sigma}\left(\mathbb{R}^{2}\right)$, com $0<\sigma<1$ e $B(., 0)$ anulando-se de ordem $2 k-1$ na abscissa $\{y=0\}$, então $\omega$ tem a forma $\omega(x, y)=e^{\left(s_{0} \circ z_{k}\right)(x, y)} \cdot\left(h \circ z_{k}\right)(x, y)$, sendo $s_{0}$ uma função Hölder contínua, $z_{k}$ a integral primeira de $L_{k}$ e $h$ uma função holomorfa. Reciprocamente, para cada função holomorfa $h$, existe uma função $s_{0}$ Hölder contínua, tal que $\omega(x, y)=e^{\left(s_{0} \circ z_{k}\right)(x, y)} \cdot\left(h \circ z_{k}\right)(x, y)$ satisfaz $[* 6]$, sendo $z_{k}$ a integral primeira de $L_{k}$.

Similarmente, se $\omega \in C^{1}$ é solução da equação

$$
L \omega=A \cdot \omega+B \cdot \bar{\omega}
$$

em um aberto conexo limitado $U$ que contem a origem, sendo $A, B \in C^{2+\sigma}\left(\mathbb{R}^{2}\right)$, com $0<\sigma<1$ e $B(0, y)=\frac{\partial B}{\partial x}(0, y)=0, \forall y \in \mathbb{R}$, então $\omega$ tem a forma $\omega(x, y)=e^{\left(s_{0} \circ z\right)(x, y)} \cdot(H \circ z)(x, y)$, sendo $s_{0}$ uma função Hölder contínua, $z$ a integral primeira de $L$ e $H$ uma função holomorfa. Reciprocamente, para cada função holomorfa $H$, existe uma função $s_{0}$ Hölder contínua, tal que $\omega(x, y)=e^{\left(s_{0} \circ z\right)(x, y)} \cdot(H \circ z)(x, y)$ satisfaz [*7], sendo $z$ a integral primeira de $L$.

Um dos objetivos deste trabalho é apresentar em detalhes a demonstração dos resultados citados acima. Também, apresentaremos resultados sobre o Princípio da Similaridade para o campo vetorial $\frac{\partial}{\partial \bar{z}}$ (ver capítulo 2$)$. 


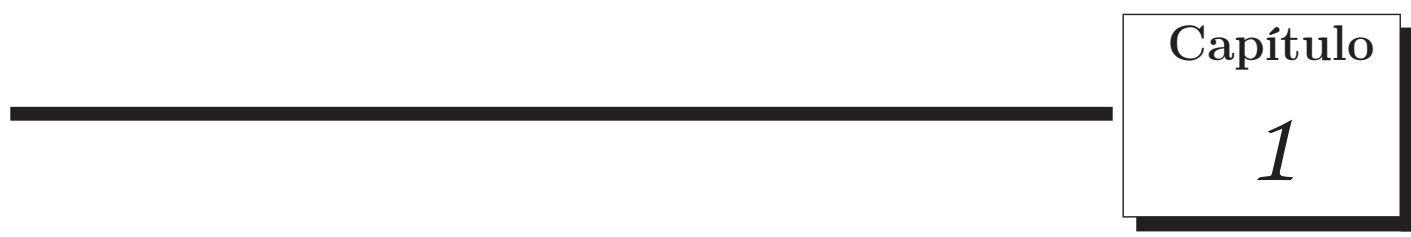

\section{Preliminares}

\subsection{Alguns resultados de Análise Real}

Definição 1.1 (Espaço Métrico). Sejam $M$ um conjunto qualquer, com $M \neq \emptyset$, e $d: M \times M \rightarrow \mathbb{R}_{0}^{+}$. A função d é chamada Métrica sobre $M$ e o par $(M, d)$ chamamos Espaço Métrico, se d satisfaz as seguintes propriedades para quaisquer $x, y, z \in M$ :

1. $d(x, y) \geq 0$ e $d(x, y)=0$ se e somente se $x=y$;

2. $d(x, y)=d(y, x)$;

3. $d(x, z) \leq d(x, y)+d(y, z)$.

Sejam $\left(X, d_{1}\right)$ e $\left(Y, d_{2}\right)$ espaços métricos. Denotamos o espaço das funções contínuas por

$$
C(X ; Y)=\{f: X \rightarrow Y ; f \text { é contínua }\}
$$

Definição 1.2. Sejam $\left(X, d_{1}\right),\left(Y, d_{2}\right)$ espaços métricos e seja $f:\left(X, d_{1}\right) \rightarrow\left(Y, d_{2}\right)$ uma função. Dizemos que $f$ é limitada se existe uma constante $K>0$ tal que

$$
d_{2}(f(x), f(y)) \leq K,
$$

para todo $x, y \in X$.

Sejam $\left(X, d_{1}\right)$ e $\left(Y, d_{2}\right)$ espaços métricos. Denotamos o espaço das funções limitadas por

$$
B(X ; Y)=\{f: X \rightarrow Y ; f \text { é limitada }\}
$$


Sejam $\left(X, d_{1}\right)$ e $\left(Y, d_{2}\right)$ espaços métricos. Denotamos o espaço das funções contínuas e limitadas por

$$
C_{0}(X ; Y)=\{f: X \rightarrow Y ; f \text { é contínua e limitada }\}
$$

Observação 1.1. O espaço $C_{0}(X ; Y)$ pode ser metrizado pela função

$$
d_{u}(f, g)=\sup _{x \in X}\left\{d_{2}(f(x), g(x))\right\}
$$

Demonstração.

Primeiro vamos mostrar que a função $d_{u}$ está bem definida. Para isso, sejam $f, g \in C_{0}(X ; Y)$; daí, existem $K_{1}, K_{2}>0$ tal que

$$
d_{2}(f(x), f(y)) \leq K_{1} \text { e } d_{2}(g(x), g(y)) \leq K_{2}
$$

para todo $x, y \in X$. Então, dado $x \in X$, tomemos $y_{0} \in X$ ponto fixado; assim temos

$$
\begin{aligned}
d_{2}(f(x), g(x)) & \leq d_{2}\left(f(x), f\left(y_{0}\right)\right)+d_{2}\left(f\left(y_{0}\right), g\left(y_{0}\right)\right)+d_{2}\left(g\left(y_{0}\right), g(x)\right) \\
& \leq K_{1}+d_{2}\left(f\left(y_{0}\right), g\left(y_{0}\right)\right)+K_{2}=K<\infty
\end{aligned}
$$

Portanto, pela definição de supremo, temos que

$$
\sup _{x \in X}\left\{d_{2}(f(x), g(x))\right\} \leq K
$$

Agora, vamos mostrar que $d_{u}$ é uma métrica. Assim, temos:

1. $d_{u}(f, g) \geq 0$.

De fato, temos que $d_{u}(f, g)=\sup _{x \in X}\left\{d_{2}(f(x), g(x))\right\} \geq d_{2}(f(x), g(x)) \geq 0$.

2. $d_{u}(f, g)=0 \Leftrightarrow f=g$.

De fato,

$(\Rightarrow)$ Temos que $0=d_{u}(f, g)=\sup _{x \in X}\left\{d_{2}(f(x), g(x))\right\} \geq d_{2}(f(x), g(x)), \forall x \in X$.

Daí, $d_{2}(f(x), g(x))=0, \forall x \in X$; logo, como $d_{2}$ é métrica, temos $f(x)=g(x)$,

$\forall x \in X$.

$(\Leftarrow)$ Temos que $f(x)=g(x), \forall x \in X$.

Daí, $d_{2}(f(x), g(x))=0, \forall x \in X$; logo, temos

$$
0=\sup _{x \in X}\left\{d_{2}(f(x), g(x))\right\}=d_{u}(f, g)
$$


3. $d_{u}(f, g)=d_{u}(g, f)$.

De fato, $d_{u}(f, g)=\sup _{x \in X}\left\{d_{2}(f(x), g(x))\right\}=\sup _{x \in X}\left\{d_{2}(g(x), f(x))\right\}=d_{u}(g, f)$.

4. $d_{u}(f, g) \leq d_{u}(f, h)+d_{u}(h, g)$.

De fato, pela desigualdade triangular, temos que

$$
d_{2}(f(x), g(x)) \leq d_{2}(f(x), h(x))+d_{2}(h(x), g(x)) .
$$

Por outro lado, temos

$d_{2}(f(x), h(x)) \leq \sup _{x \in X}\left\{d_{2}(f(x), h(x))\right\}$ e $d_{2}(h(x), g(x)) \leq \sup _{x \in X}\left\{d_{2}(h(x), g(x))\right\}$.

Logo,

$d_{2}(f(x), g(x)) \leq \sup _{x \in X}\left\{d_{2}(f(x), h(x))\right\}+\sup _{x \in X}\left\{d_{2}(h(x), g(x))\right\}$.

Assim, pela definição de supremo, temos que

$$
\sup _{x \in X}\left\{d_{2}(f(x), g(x))\right\} \leq \sup _{x \in X}\left\{d_{2}(f(x), h(x))\right\}+\sup _{x \in X}\left\{d_{2}(h(x), g(x))\right\} .
$$

Portanto, $d_{u}(f, g) \leq d_{u}(f, h)+d_{u}(h, g)$.

Finalmente, temos que $\left(C_{0}(X ; Y), d_{u}\right)$ é um espaço métrico.

Observação 1.2. Se $X$ é compacto então $C_{0}(X ; Y)=C(X ; Y)$.

Definição 1.3 (Espaço Normado). Sejam E um espaço vetorial sobre o corpo $\mathbb{K}(\mathbb{R}$ ou $\mathbb{C}) e\|\|:. E \rightarrow \mathbb{R}_{0}^{+}$. A função $\|$.$\| é chamada Norma e o par (E,\|\|$.$) é chamado Espaço$ Normado, se \|.\| satisfaz as seguintes propriedades para quaisquer $x, y \in E$ e $\lambda \in \mathbb{K}$ :

1. $\|x\| \geq 0$ e $\|x\|=0$ se e somente se $x=0$;

2. $\|\lambda x\|=|\lambda|\|x\|$;

3. $\|x+y\| \leq\|x\|+\|y\|$.

Observação 1.3. Todo espaço vetorial normado (E, $\|\|$.$) torna-se um espaço métrico por$ meio da definição

$$
d(x, y)=\|x-y\| .
$$

Esta métrica diz-se proveniente da norma $\|$.$\| .$

Além das propriedades requeridas para uma métrica (as quais se verificam facilmente a partir da definição de norma), a distância definida por uma norma satisfaz ainda: $d(x+z, y+z)=d(x, y)$ e $d(\lambda x, \lambda y)=|\lambda| \cdot d(x, y), \forall x, y, z \in E, \lambda \in \mathbb{K}(\mathbb{R}$ ou $\mathbb{C})$. 
Observação 1.4. Seja $X \neq \emptyset$ um conjunto qualquer. Se $\left(E,\|\cdot\|_{E}\right)$ é um espaço vetorial normado então o conjunto $B(X ; E)$ possui uma estrutura natural de espaço vetorial, i.e, $\forall f, g \in B(X ; E), \lambda \in \mathbb{K}(\mathbb{R}$ ou $\mathbb{C})$, temos:

$$
\begin{aligned}
(f+g)(x) & =f(x)+g(x) \\
(\lambda \cdot f)(x) & =\lambda \cdot f(x)
\end{aligned}
$$

para todo $x \in X$.

Proposição 1.1. Seja $X \neq \emptyset$ um conjunto qualquer e $\left(Y,\|\cdot\|_{Y}\right)$ um espaço vetorial normado. O subespaço vetorial $C_{0}(X ; Y) \subset B(X ; Y)$ pode ser normado por

$$
\|f\|_{u}=\sup _{x \in X}\|f(x)\|_{Y}
$$

$\forall f \in C_{0}(X ; Y)$.

Além disso, $C_{0}(X ; Y)$ torna-se um espaço métrico por meio da definição

$$
d_{u}(f, g)=\|f-g\|_{u}
$$

Demonstração.

De fato, como $f$ é limitada, temos que $\|f\|_{u}<\infty$.

Mostremos que $\|\cdot\|_{u}$ é uma norma. Assim, temos:

- $\|f\|_{u}=0$ se, e somente se, $f(x)=0$, para todo $x \in X$;

- $\|\lambda f\|_{u}=|\lambda| \cdot\|f\|_{u}$

De fato, $\|\lambda f\|_{u}=\sup _{x \in X}\|\lambda f(x)\|_{Y}=|\lambda| \cdot \sup _{x \in X}\|f(x)\|_{Y}=|\lambda| \cdot\|f\|_{u}$.

- $\|f+g\|_{u} \leq\|f\|_{u}+\|g\|_{u}$;

De fato, seja $g \in C_{0}(X ; Y)$. Definimos $h(x)=f(x)+g(x), \forall x \in X$, sendo $f \in C_{0}(X ; Y)$; logo, $h \in C_{0}(X ; Y)$. Daí, pela desigualdade triangular e por propriedade do supremo temos

$$
\|h(x)\|_{Y} \leq\|f(x)\|_{Y}+\|g(x)\|_{Y} \leq \sup \|f(x)\|_{Y}+\sup \|g(x)\|_{Y}=\|f\|_{u}+\|g\|_{u},
$$

para qualquer $x \in X$. Assim, pela definição de supremo, temos que

$$
\sup \|h(x)\|_{Y} \leq\|f\|_{u}+\|g\|_{u}
$$


Portanto,

$$
\|f+g\|_{u} \leq\|f\|_{u}+\|g\|_{u}
$$

Assim, a função $\|\cdot\|_{u}: C_{0}(X ; Y) \rightarrow \mathbb{R}_{0}^{+}$é uma norma. Finalmente, $\left(C_{0}(X ; Y),\|\cdot\|_{u}\right)$ é um espaço normado.

Agora, para todo $f, g \in C_{0}(X ; Y)$, as propriedades requeridas para que $d_{u}$ seja uma métrica se verificam facilmente a partir da definição de norma.

Portanto, $\left(C_{0}(X ; Y), d_{u}\right)$ é um espaço métrico.

Definição 1.4 (Espaço de Banach). Um espaço normado que é completo com a métrica induzida pela norma é chamado espaço de Banach.

Teorema 1.1. Sejam $\left(X, d_{1}\right)$ e $\left(Y, d_{2}\right)$ espaços métricos. Se o espaço $\left(Y, d_{2}\right)$ é completo então $\left(C_{0}(X ; Y), d_{u}\right)$ é completo.

Demonstração.

De fato, seja $\left(f_{n}\right)_{n \geq 1}$ uma seqüência de Cauchy em $C_{0}(X ; Y)$. Para cada $x \in X$, temos que $\left(f_{n}(x)\right)_{n \geq 1}$ é uma seqüência de Cauchy em $Y$ e como $Y$ é completo então $\left(f_{n}(x)\right)_{n \geq 1}$ converge em $\mathrm{Y}$.

Definamos $F: X \rightarrow Y$ por

$$
F(x)=\lim _{n \rightarrow \infty} f_{n}(x)
$$

Mostremos que $\left(f_{n}\right)_{n \geq 1}$ converge uniformemente a $F$, isto é, para todo $\epsilon>0, \exists N$ tal que se $n \geq N, d_{2}\left(f_{n}(x), F(x)\right)<\epsilon$, para todo $x \in X$.

De fato, como $\left(f_{n}\right)_{n \geq 1}$ é de Cauchy, temos que dado $\epsilon>0$, escolhemos $N>0$ tal que se $n, m \geq N$ então $d_{u}\left(f_{n}(x), f_{m}(x)\right)<\epsilon / 2$, para todo $x \in X$.

Daí, por propriedade do supremo temos

$$
d_{2}\left(f_{n}(x), f_{m}(x)\right) \leq \sup _{x \in X}\left\{d_{2}\left(f_{n}(x), f_{m}(x)\right)\right\}=d_{u}\left(f_{n}(x), f_{m}(x)\right)<\epsilon, \forall x \in X
$$

assim, para todo $x \in X$ temos

$$
d_{2}\left(f_{n}(x), f_{m}(x)\right)<\epsilon / 2
$$

Afirmação: $d_{2}\left(f_{n}(x), F(x)\right)<\epsilon$, para todo $x \in X$, se $n \geq N$.

De fato, seja $x_{0} \in X$ um ponto qualquer. Como a seqüência $\left(f_{n}\left(x_{0}\right)\right)_{n \geq 1}$ converge a $F\left(x_{0}\right)$; então, existe $N_{0}>0$ tal que

$$
d_{2}\left(f_{n}\left(x_{0}\right), F\left(x_{0}\right)\right)<\epsilon / 2
$$


para todo $n \geq N_{0}$ ( $N_{0}$ depende de $x_{0}$, mas isto não é problema já que $N$ não depende de $x_{0}$ ). Escolhemos um $n_{0} \in \mathbb{Z}^{+}$tal que $n_{0}>\max \left\{N, N_{0}\right\}$. Assim, se $n \geq N$, temos que

$$
d_{2}\left(f_{n}\left(x_{0}\right), F\left(x_{0}\right)\right) \leq d_{2}\left(f_{n}\left(x_{0}\right), f_{n_{0}}\left(x_{0}\right)\right)+d_{2}\left(f_{n_{0}}\left(x_{0}\right), F\left(x_{0}\right)\right)<\epsilon / 2+\epsilon / 2=\epsilon .
$$

Como $x_{0}$ foi tomado arbitrário, temos que $d_{2}\left(f_{n}(x), F(x)\right)<\epsilon, \forall x \in X$. Logo, como $N$ não depende de $x_{0}$, podemos concluir que $\left(f_{n}\right)_{n \geq 1}$ converge uniformemente a $F$.

Agora, mostremos que $F \in C_{0}(X ; Y)$, isto é, dado $\epsilon>0, \exists \delta>0$ tal que $x \in X$, $d_{1}\left(x, x_{0}\right)<\delta$, então $d_{2}\left(F(x), F\left(x_{0}\right)\right)<\epsilon$.

De fato, como $d_{2}$ é contínua temos

$$
\begin{aligned}
d_{2}\left(F(x), F\left(x_{0}\right)\right) & =\lim _{n \rightarrow \infty} d_{2}\left(f_{n}(x), F\left(x_{0}\right)\right) \\
& \leq \lim _{n \rightarrow \infty}\left\{d_{2}\left(f_{n}(x), f_{n}\left(x_{0}\right)\right)+d_{2}\left(f_{n}\left(x_{0}\right), F\left(x_{0}\right)\right)\right\}
\end{aligned}
$$

Por outro lado, sabemos que $\left(f_{n}\right)_{n \geq 1} \in C_{0}(X ; Y)$; daí, as $\left(f_{n}\right)_{n \geq 1}$ são contínuas em $x_{0} \in X$; assim, dado $\epsilon / 2>0$ escolha $\delta>0$ como acima e como $d_{1}\left(x, x_{0}\right)<\delta$; então, temos que $d_{2}\left(f_{n}(x), f_{n}\left(x_{0}\right)\right)<\epsilon / 2$, para todo $n \geq 1$.

Logo, substituindo $(* 1)$ em $(* 2)$, temos que

$$
d_{2}\left(F(x), F\left(x_{0}\right)\right)<\lim _{n \rightarrow \infty}\{\epsilon / 2+\epsilon / 2\}=\epsilon \text {. }
$$

Finalmente, mostremos que $F$ é limitada, isto é, $\exists c>0$ tal que $d_{2}(F(x), F(y)) \leq c$, $\forall x, y \in X$.

De fato, sejam $x, y \in X$ e $\left(f_{n}\right)_{n \geq 1}$ uma seqüência de Cauchy em $C_{0}(X ; Y)$; logo, temos que $\left(f_{n}\right)_{n \geq 1}$ é limitada em $C_{0}(X ; Y)$, i.e, existe $f \in C_{0}(X ; Y)$ e $M_{1}>0$ tal que

$$
d_{u}\left(f_{n}, f\right) \leq M_{1}, \forall n \geq 1
$$

Daí, $d_{2}\left(f_{n}(x), f(x)\right) \leq \sup _{x \in X}\left\{d_{2}\left(f_{n}(x), f(x)\right)\right\} \leq d_{u}\left(f_{n}, f\right) \leq M_{1}, \forall n \geq 1$ e para todo $x \in X$; logo, para todo $x \in X$ temos

$$
d_{2}\left(f_{n}(x), f(x)\right) \leq M_{1}, \forall n \geq 1
$$

Por outro lado, como $f$ é limitada em $Y$, temos que existe $M_{2}>0$ tal que

$$
d_{2}(f(x), f(y)) \leq M_{2}, \forall x, y \in X
$$


Agora, dados $x, y \in X$, pela desigualdade triangular e de $(* 3)$ e $(* 4)$, temos que

$$
\begin{aligned}
d_{2}(F(x), F(y)) & \leq d_{2}(F(x), f(x))+d_{2}(f(x), f(y))+d_{2}(f(y), F(y)) \\
& \leq \lim _{n \rightarrow \infty} d_{2}\left(f_{n}(x), f(x)\right)+d_{2}(f(x), f(y))+\lim _{n \rightarrow \infty} d_{2}(f(y), F(y)) \\
& \leq \lim _{n \rightarrow \infty} M_{1}+M_{2}+\lim _{n \rightarrow \infty} M_{1} \\
& \leq 2 M_{1}+M_{2}=C,
\end{aligned}
$$

sendo $C=2 M_{1}+M_{2}>0$.

Portanto, podemos concluir que $F$ é limitada. Finalmente, $\left(C_{0}(X ; Y), d_{u}\right)$ é um espaço métrico completo.

Observação 1.5. Se $\left(Y,\|.\|_{Y}\right)$ é um espaço de Banach, temos pelo teorema 1.1 que o espaço $\left(C_{0}(X ; Y),\|.\|_{u}\right)$ é um espaço de Banach.

Definição 1.5. Seja $\left(f_{j}\right)_{j \in \mathbb{N}}$ uma seqüência de funções, com $f_{j}: X \subset \mathbb{R}^{m} \rightarrow \mathbb{R}^{n}, \forall j \in \mathbb{N}$. Dizemos que $\left(f_{j}\right)_{j \in \mathbb{N}}$ é uniformemente limitada, se existe $c>0$ tal que $\left|f_{j}(x)\right| \leq c$, para todo $j \in \mathbb{N} e \forall x \in X$.

Definição 1.6. Seja $\left(f_{j}\right)_{j \in \mathbb{N}}$ uma seqüência de funções, com $f_{j}: X \subset \mathbb{R}^{m} \rightarrow \mathbb{R}^{n}, \forall j \in \mathbb{N}$. Dizemos que $\left(f_{j}\right)_{j \in \mathbb{N}}$ converge uniformemente para uma aplicação $f: X \rightarrow \mathbb{R}^{n}$, se para qualquer $\epsilon>0$ dado, pode-se obter $j_{0} \in \mathbb{N}$ tal que $j>j_{0}$ então $\left|f_{j}(x)-f(x)\right|<\epsilon$, seja qual for $x \in X$.

Definição 1.7. Seja $f: X \rightarrow \mathbb{R}^{n}$ uma aplicação definida em o aberto $X \subset \mathbb{R}^{m}$. Dizemos que $f$ é uniformemente contínua quando, para todo $\epsilon>0$ dado, pode-se obter $\delta>0$ tal que se $x, y \in X,|x-y|<\delta$ então $|f(x)-f(y)|<\epsilon$.

Definição 1.8. Seja $\left(f_{j}\right)_{j \in \mathbb{N}}$ uma família de funções contínuas, com $f_{j}: X \subset \mathbb{R}^{m} \rightarrow \mathbb{R}^{n}$, $\forall j \in \mathbb{N}$. Dizemos que a família é eqüicontínua em $X$ se $\forall \epsilon>0, \exists \delta(\epsilon)>0$ tal que $\forall x, y \in X,|x-y|<\delta$ então $\left|f_{j}(x)-f_{j}(y)\right|<\epsilon$, para qualquer $j \in \mathbb{N}$.

Teorema 1.2 (Ascoli-Arzelá). Seja $\left(f_{j}\right)_{j \in \mathbb{N}}$ uma familia de funções contínuas, com $f_{j}: X \rightarrow \mathbb{R}^{n}, \forall j \in \mathbb{N}$, definidas em $X \subset \mathbb{R}^{m}$ e seja $\bar{X}$ compacto. Se $\left(f_{j}\right)_{j \in \mathbb{N}}$ é eqüicontínuo e uniformemente limitado. Então, $\left(f_{j}\right)_{j \in \mathbb{N}}$ possui uma subseqüência $\left(f_{j_{k}}\right)_{j_{k} \in \mathbb{N}}$ que converge uniformemente a uma função contínua $f: X \rightarrow \mathbb{R}^{n}$.

A demonstração deste teorema pode ser encontrado em [5]. 
Definição 1.9. Seja $(X, d)$ um espaço métrico. Dizemos que $X$ é compacto, se toda seqüência $\left(x_{n}\right)_{n \in \mathbb{N}}$ em $X$ possui uma subseqüência $\left(x_{n_{k}}\right)_{n_{k} \in \mathbb{N}}$ que converge para um elemento de $X$.

Definição 1.10. Seja $E$ um espaço vetorial. Dizemos que $K \subset E$ é convexo, se para todo $x, y \in X \quad e \forall \lambda \in[0,1],(1-\lambda) x+\lambda y \in K$.

Teorema 1.3 (Ponto fixo de Shauder). Seja $X$ um espaço de Banach, $K \subset X$ um conjunto compacto e convexo. Se $T: K \rightarrow K$ é uma função contínua então $T$ admite um ponto fixo $x \in K$, ou seja, $T x=x$.

A demonstração deste teorema pode ser encontrado em [6].

\subsection{Alguns resultados de Análise Complexa}

Teorema 1.4. Suponhamos que a função $f(z)=u(z)+i v(z)$ está definida em um aberto $\Omega \subset \mathbb{C}$, sendo $u, v$ funções a valores reais e que as derivadas parciais $\frac{\partial u}{\partial x}, \frac{\partial u}{\partial y}, \frac{\partial v}{\partial x}, \frac{\partial v}{\partial y}$ existem em todo ponto de $\Omega$. Se cada uma dessas derivadas parciais é contínua em um ponto $z_{0} \in \Omega$ e se as equações Cauchy-Riemann são satisfeitas por u e $v$ em $z_{0}$, então $f$ é diferenciável em $z_{0}$.

A demonstração deste teorema pode ser encontrado em [7].

Definição 1.11 (Singularidade isolada). Seja $f$ uma função complexa. Dizemos que $f$ tem uma singularidade isolada em $z_{0} \in \mathbb{C}$, se existe $r>0$ tal que $f$ é analítica em $D\left(z_{0}, r\right) \backslash\left\{z_{0}\right\}$, mas não é analítica em $z_{0}$.

Observação 1.6. Seja $f$ uma função analítica em $D\left(z_{0}, r\right) \backslash\left\{z_{0}\right\}$. Existem duas alternativas para que $f$ tenha uma singularidade isolada em $z_{0}$ : ou $z_{0}$ não pertence ao domínio de $f$ ou $z_{0}$ pertence ao domínio de $f$, mas é um ponto no qual $f$ é descontínua.

Exemplo 1.1. A primeira alternativa da observação é exemplificada pela função $f(z)=\frac{1}{z}$ no ponto $z_{0}=0$. A segunda é exemplificada pela função

$$
f(z)=\left\{\begin{array}{ll}
0 & \text { se } z \neq 0 \\
1 & \text { se } z=0
\end{array}\right. \text {, }
$$

no ponto $z_{0}=0$.

Definição 1.12 (Singularidade removível). Seja $f$ uma função complexa e $z_{0} \in \mathbb{C}$ uma singularidade isolada de $f$. Dizemos que $f$ tem uma singularidade removível em $z_{0}$, se existe uma função analítica $g: D\left(z_{0}, r\right) \rightarrow \mathbb{C}$ tal que $g(z)=f(z), \forall z \in D\left(z_{0}, r\right) \backslash\left\{z_{0}\right\}$. 
Teorema 1.5. Suponhamos que $f$ seja uma função complexa analítica e não identicamente nula em uma região $\Omega$ e que $z_{0} \in \Omega$ é um zero de $f$. Então, existe $D\left(z_{0}, r\right)$ com $r>0$ tal que $f(z) \neq 0, \forall z \in D\left(z_{0}, r\right) \backslash\left\{z_{0}\right\}$.

A demonstração deste teorema pode ser encontrado em [7].

Teorema 1.6. Suponhamos que uma função complexa $f$ tem uma singularidade isolada em um ponto $z_{0}$. A singularidade é removível se, e somente se, $f\left(z_{0}\right)$ pode ser definido (ou redefinido ) de modo a tornar $f$ analítica em $z_{0}$.

A demonstração deste teorema pode ser encontrado em [7].

Teorema 1.7. Seja $f$ uma função complexa. Se $f$ tem uma singularidade isolada em $z_{0}$. Então, o ponto $z=z_{0}$ é uma singularidade removivel se, e somente se,

$$
\lim _{z \rightarrow z_{0}}\left(z-z_{0}\right) f(z)=0
$$

A demonstração deste teorema pode ser encontrado em [4].

Teorema 1.8 (De Extensão de Riemann). Suponhamos que uma função complexa $f$ tem uma singularidade isolada em um ponto $z_{0}$. A singularidade é removível se, e somente se, $f$ é limitada em algum $D\left(z_{0}, r\right) \backslash\left\{z_{0}\right\}$, com $r>0$.

A demonstração deste teorema pode ser encontrado em [7].

\subsection{Elementos básicos sobre distribuições}

Definição 1.13 (Suporte de uma função). Seja $M$ um espaço topológico e $V$ um espaço vetorial. O suporte de uma função $f: M \rightarrow V$ é o conjunto

$$
\operatorname{Supp}(f)=\overline{\{x \in M: f(x) \neq 0\}} \text {. }
$$

Isso significa que, se $x \in M$ é um ponto fora do suporte de $f$ então $f$ é nula numa vizinhança de $x$.

Definição 1.14 (Suporte compacto de uma função). Seja $M$ um espaço topológico e $V$ um espaço vetorial. Dizemos que uma função $f: M \rightarrow V$ tem suporte compacto, se existe um conjunto compacto $K \subset M$ tal que $f$ se anula em $M \backslash K$. 
Definição 1.15 (O espaço das funções testes). Seja $\Omega$ um subconjunto aberto de $\mathbb{R}^{n}$. O conjunto

$$
C_{c}^{\infty}(\Omega)=\left\{\phi: \Omega \rightarrow \mathbb{C} ; \phi \in C^{\infty}(\Omega) \text { e Supp }(\phi) e ́ \text { compacto }\right\}
$$

é chamado espaço das funções testes.

Observação 1.7. O conjunto das funções de classe $C^{k}(\Omega)$ possuindo suporte compacto será indicado por $C_{c}^{k}(\Omega)$, para $k \geq 1$.

Definição 1.16 (Convergência em $C_{c}^{\infty}(\Omega)$ ). Seja $\Omega$ um subconjunto aberto de $\mathbb{R}^{n}$. Dizemos que a seqüência $\left(\phi_{j}\right)_{j \in \mathbb{N}} \subset C_{c}^{\infty}(\Omega)$ converge a zero em $C_{c}^{\infty}(\Omega)$ se:

1. existe um subconjunto compacto $K \subset \Omega$ tal que $\operatorname{Supp}\left(\phi_{j}\right) \subset K, \forall j=1,2, \cdots$ e

2. para cada $\alpha=\left(\alpha_{1}, \alpha_{2}, \cdots, \alpha_{n}\right)$, $\partial^{\alpha} \phi_{j}$ converge uniformemente a zero em $K$.

Definição 1.17 (Distribuição). Consideremos $\Omega$ um subconjunto aberto de $\mathbb{R}^{n}$. Um funcional linear $\mu: C_{c}^{\infty}(\Omega) \rightarrow \mathbb{C}$ continuo denomina-se distribuição.

Observação 1.8. Denotaremos o valor da distribuição $\mu$ na função teste $\phi \in C_{c}^{\infty}(\Omega)$ por $\langle\mu, \phi\rangle$.

Observação 1.9. Indicaremos por $\mathcal{D}^{\prime}(\Omega)$ o conjunto das distribuições definidas em $C_{c}^{\infty}(\Omega)$.

Observação 1.10. Levando em conta que qualquer funcional linear em $C_{c}^{\infty}(\Omega)$ é contínuo se, e somente se, ele for contínuo no ponto zero, então podemos dizer que o funcional linear $\mu: C_{c}^{\infty}(\Omega) \rightarrow \mathbb{C}$ é contínuo se, e somente se, para qualquer seqüência $\left(\varphi_{j}\right)_{j \in \mathbb{N}} \subset C_{c}^{\infty}(\Omega)$ convergindo a zero em $C_{c}^{\infty}(\Omega)$, temos que $\mu\left(\varphi_{j}\right)$ converge a zero em $\mathbb{C}$.

Definição 1.18 (Operações com distribuições). Sejam S,T distribuições arbitrárias e c, $x \in \mathbb{R}^{n}$, podemos definir de forma natural novas distribuições tais como $S+T, c \cdot T$, $\partial^{\alpha} T$, sendo $\alpha$ um multiindice, $T(c x)(c \neq 0), T(x-c), g(x) \cdot T(x), \forall g \in C^{\infty}$. Assim, para todo $\varphi \in C_{c}^{\infty}(\Omega)$ temos:

$$
\begin{aligned}
& \text { 1. }\langle S+T, \varphi\rangle=\langle S, \varphi\rangle+\langle T, \varphi\rangle ; \\
& \text { 2. }\langle c \cdot T, \varphi\rangle=c \cdot\langle T, \varphi\rangle ; \\
& \text { 3. }\left\langle\partial^{\alpha} T, \varphi\right\rangle=(-1)^{|\alpha|}\left\langle T, \partial^{\alpha} \varphi\right\rangle ; \\
& \text { 4. }\langle T(c x), \varphi\rangle=|c|^{-1}\langle T, \varphi(x / c)\rangle, \forall c \neq 0 ;
\end{aligned}
$$


5. $\langle T(x-c), \varphi\rangle=\langle T, \varphi(x+c)\rangle$;

6. $\langle g(x) T(x), \varphi\rangle=\langle T, g(x) \varphi(x)\rangle$.

Definição 1.19 (Suporte de uma distribuição). O suporte da distribuição $\mu$ de $\mathcal{D}^{\prime}(\Omega)$, indicado por $\operatorname{Supp}(\mu)$, é a interseção de todos os fechados fora dos quais $\mu=0$, isto é, $\langle\mu, \varphi\rangle=0$, para cada $\varphi \in C_{c}^{\infty}(\Omega \backslash \operatorname{Supp}(\mu))$.

Definição 1.20 ( $\mathrm{O}$ espaço $\mathcal{E}^{\prime}(\Omega)$ ). O conjunto das distribuições com suporte compacto será denotado por $\mathcal{E}^{\prime}(\Omega)$. Isto equivale a

$$
\mathcal{E}^{\prime}(\Omega)=\left\{\mu \in \mathcal{D}^{\prime}(\Omega): \operatorname{Supp}(\mu) \text { é compacto }\right\}
$$

Observação 1.11. Seja $\Omega$ um subconjunto aberto de $\mathbb{R}^{n}$, temos $C_{c}^{\infty}(\Omega) \subset \mathcal{E}^{\prime}(\Omega) \subset \mathcal{D}^{\prime}(\Omega)$.

\subsubsection{Alguns fatos sobre convoluções}

Definição 1.21. Sejam $f, g$ funções contínuas em $\mathbb{R}^{n}$, uma das quais tem suporte compacto. Definimos a convolução

$$
(f * g)(x)=\int_{\mathbb{R}^{n}} f(x-y) g(y) d y=\int_{\mathbb{R}^{n}} f(y) g(x-y) d y
$$

$\forall x, y \in \mathbb{R}^{n}$

Segue que, $f * g=g * f$.

Também, observamos que em linguagem de distribuições temos

$$
(f * g)(x)=\left\langle f, \breve{g_{x}}\right\rangle,
$$

sendo $\breve{g_{x}}(y)=g(x-y), \forall x, y \in \mathbb{R}^{n}$.

Além das regras acima, não é difícil verificar que se $f$ e $g$ forem diferenciáveis em relação a variável $x_{j}, \forall j=1 \ldots n$, então

$$
\frac{\partial}{\partial x_{j}}(f * g)=\frac{\partial f}{\partial x_{j}} * g=f * \frac{\partial g}{\partial x_{j}} .
$$

Se $f, g \in C^{k}$ e $|\alpha| \leq k$, com $k=1,2, \cdots$, por um processo indutivo temos

$$
\partial^{\alpha}(f * g)=\left(\partial^{\alpha} f\right) * g=f *\left(\partial^{\alpha} g\right),
$$


$\forall \alpha \in \mathbb{Z}_{+}^{n}$, desde que estas expressões façam sentido.

Veremos a seguir como estas noções estendem-se para distribuições.

Definição 1.22 (Convolução de distribuição com função). Sejam $\mu \in \mathcal{D}^{\prime}\left(\mathbb{R}^{n}\right) e$ $\varphi \in C_{c}^{\infty}\left(\mathbb{R}^{n}\right)$ (ou $\mu \in \mathcal{E}^{\prime}\left(\mathbb{R}^{n}\right)$ e $\left.\varphi \in C^{\infty}\left(\mathbb{R}^{n}\right)\right)$. A convolução de $\mu$ com $\varphi$ é a função indicada por $\mu * \varphi$; a qual calculada no ponto a é $\left\langle\mu, \breve{\varphi}_{a}\right\rangle$, ou seja, para cada a $\in \mathbb{R}^{n}$, temos

$$
(\mu * \varphi)(a)=\left\langle\mu, \breve{\varphi}_{a}\right\rangle
$$

sendo $\breve{\varphi}_{a}(x)=\varphi(a-x), \forall x, a \in \mathbb{R}^{n}$.

Teorema 1.9. Dados $\mu \in \mathcal{D}^{\prime}\left(\mathbb{R}^{n}\right)$ e $\varphi \in C_{c}^{\infty}\left(\mathbb{R}^{n}\right)\left(\right.$ ou $\left.\mu \in \mathcal{E}^{\prime}\left(\mathbb{R}^{n}\right) e \varphi \in C^{\infty}\left(\mathbb{R}^{n}\right)\right)$, a função $\mu * \varphi$ é de classe $C^{\infty}$. Além disso, dado qualquer multiíndice $\alpha$, valem as igualdades

$$
\partial^{\alpha}(\mu * \varphi)=\left(\partial^{\alpha} \mu\right) * \varphi=\mu *\left(\partial^{\alpha} \varphi\right)
$$

Demonstração.

Seja $a \in \mathbb{R}^{n}, j \in\{1,2, \ldots, n\}$ e $\left(h_{k}\right)_{k \in \mathbb{N}} \subset \mathbb{R}$ uma seqüência de termos não nulos convergindo a zero. Então, aplicando o teorema do Valor Médio podemos escrever

$$
\begin{aligned}
\frac{\breve{\varphi}_{a+h_{k} \cdot e_{j}}-\breve{\varphi}_{a}}{h_{k}}-\frac{\breve{\partial \varphi}}{\partial x_{j}}(a) & =\frac{1}{h_{k}}\left[\varphi\left(a-x+h_{k} \cdot e_{j}\right)-\varphi(a-x)\right]-\frac{\partial \varphi}{\partial x_{j}}(a-x) \\
& =\frac{\partial \varphi}{\partial x_{j}}\left(a-x+\theta_{1}(a-x) h_{k} \cdot e_{j}\right)-\frac{\partial \varphi}{\partial x_{j}}(a-x) \\
& =h_{k} \cdot \theta_{1}(a-x) \cdot \frac{\partial^{2} \varphi}{\partial x_{j}^{2}}\left(a-x+\theta_{2}(a-x) \cdot \theta_{1}(a-x) \cdot h_{k} \cdot e_{j}\right),
\end{aligned}
$$

onde $\theta_{1}(a-x), \theta_{2}(a-x) \in(0,1)$. Assim,

$$
\left|\frac{\breve{\varphi}_{a+h_{k} \cdot e_{j}}-\breve{\varphi}_{a}}{h_{k}}-\frac{\breve{\partial \varphi}}{\partial x_{j}}(a)\right| \leq C(\varphi)\left|h_{k}\right| \rightarrow 0 \text {, quando } k \rightarrow \infty,
$$

com $C(\varphi)$ uma constante que só depende de $\varphi$.

No entanto, agindo como acima, podemos demonstrar que, de fato, $\frac{\breve{\varphi}_{a+h_{k} \cdot e_{j}}-\breve{\varphi}_{a}}{h_{k}} \rightarrow \frac{\breve{\partial}}{\partial x_{j}}(a)$ em $C_{c}^{\infty}\left(\mathbb{R}^{n}\right)\left(\right.$ ou em $\left.C^{\infty}\left(\mathbb{R}^{n}\right)\right)$ de acordo com $\mu \in \mathcal{D}^{\prime}\left(\mathbb{R}^{n}\right)\left(\right.$ ou em $\left.\mathcal{E}^{\prime}\left(\mathbb{R}^{n}\right)\right)$. 
Por conseguinte,

$$
\begin{aligned}
\frac{\partial(\mu * \varphi)}{\partial x_{j}}(a) & =\lim _{h_{k} \rightarrow 0} \frac{(\mu * \varphi)\left(a+h_{k} \cdot e_{j}\right)-(\mu * \varphi)(a)}{h_{k}} \\
& =\lim _{h_{k} \rightarrow 0}\left\langle\mu, \frac{\breve{\varphi}_{a+h_{k} \cdot e_{j}}-\breve{\varphi}_{a}}{h_{k}}\right\rangle=\left\langle\mu, \frac{\partial \breve{\varphi}}{\partial x_{j}}(a)\right\rangle \\
& =\left(\mu * \frac{\partial \varphi}{\partial x_{j}}\right)(a) .
\end{aligned}
$$

Agora, por um processo de indução em $|\alpha|$, obtemos $\partial^{\alpha}(\mu * \varphi)=\mu * \partial^{\alpha} \varphi$. Quanto a outra igualdade temos,

$$
\begin{aligned}
\left(\frac{\partial \mu}{\partial x_{j}} * \varphi\right)(a) & =\left\langle\frac{\partial \mu}{\partial x_{j}}, \breve{\varphi}_{a}\right\rangle=-\left\langle\mu, \frac{\partial(\varphi(a-x))}{\partial x_{j}}\right\rangle \\
& =\left\langle\mu, \frac{\partial \varphi}{\partial x_{j}}(a-x)\right\rangle=\left(\mu * \frac{\partial \varphi}{\partial x_{j}}\right)(a) .
\end{aligned}
$$

Novamente, um processo de indução nos leva a $\partial^{\alpha} \mu * \varphi=\mu * \partial^{\alpha} \varphi$.

Lema 1.1. Dados $\Omega \subset \mathbb{R}^{2}, \mu \in L^{1}(\Omega)$ e $\nu \in C_{c}^{1}(\Omega)$. Seja

$$
(\mu * \nu)(z)=\iint_{\Omega} \mu(z-\zeta) \cdot \nu(\zeta) d \xi d \eta
$$

Então,

$$
\frac{\partial}{\partial x}(\mu * \nu)(z)=\left(\frac{\partial \mu}{\partial x} * \nu\right)(z)=\left(\mu * \frac{\partial \nu}{\partial x}\right)(z)
$$

$e$

$$
\frac{\partial}{\partial y}(\mu * \nu)(z)=\left(\frac{\partial \mu}{\partial y} * \nu\right)(z)=\left(\mu * \frac{\partial \nu}{\partial y}\right)(z)
$$

Demonstração. A prova segue do teorema 1.9. 


\subsection{Sistemas não homogêneos de Cauchy-Riemann}

Sejam $\Omega$ um subconjunto aberto de $\mathbb{R}^{2}, \mu, \nu \in C^{1}(\bar{\Omega} ; \mathbb{R})$ e $g, h \in C^{1}(\bar{\Omega} ; \mathbb{R})$ funções reais conhecidas de variáveis reais $x$ e $y$. Consideremos o sistema de equações não homogêneo de Cauchy-Riemann

$$
\left\{\begin{array}{l}
\frac{\partial \mu}{\partial x}-\frac{\partial \nu}{\partial y}=g(x, y) \\
\frac{\partial \mu}{\partial y}+\frac{\partial \nu}{\partial x}=h(x, y)
\end{array}\right.
$$

Sejam $\omega(x, y)=\mu(x, y)+i \nu(x, y)$ e $f(x, y)=\frac{1}{2}(g(x, y)+i h(x, y))$, as quais são funções complexas de variáveis reais $x$ e $y$.

Os operadores

$$
\frac{\partial}{\partial z}=\frac{1}{2}\left(\frac{\partial}{\partial x}-i \frac{\partial}{\partial y}\right) \quad e \frac{\partial}{\partial \bar{z}}=\frac{1}{2}\left(\frac{\partial}{\partial x}+i \frac{\partial}{\partial y}\right)
$$

são chamados: operador de Cauchy-Riemann e anti-operador de Cauchy-Riemann, respectivamente.

Também, temos

$$
\begin{aligned}
& \frac{\partial \omega}{\partial z}=\frac{1}{2}\left(\frac{\partial \omega}{\partial x}-i \frac{\partial \omega}{\partial y}\right), \\
& \frac{\partial \omega}{\partial \bar{z}}=\frac{1}{2}\left(\frac{\partial \omega}{\partial x}+i \frac{\partial \omega}{\partial y}\right) .
\end{aligned}
$$

Assim, do sistema (1.1), temos:

$$
\frac{\partial \omega}{\partial \bar{z}}=f
$$

Também, as derivadas com respeito as variáveis $x$ e $y$, podem ser relacionadas a $\frac{\partial \omega}{\partial z}$ e $\frac{\partial \omega}{\partial \bar{z}} ;$ isto é,

$$
\begin{aligned}
& \frac{\partial \omega}{\partial x}=\frac{\partial \omega}{\partial z}+\frac{\partial \omega}{\partial \bar{z}} \\
& \frac{\partial \omega}{\partial y}=i\left(\frac{\partial \omega}{\partial z}-\frac{\partial \omega}{\partial \bar{z}}\right) .
\end{aligned}
$$

Se $\Phi$ é uma função analítica então temos $\frac{\partial \Phi}{\partial \bar{z}}=0$. 


\subsection{Fórmula integral de Cauchy não homogênea}

Proposição 1.2. Sejam $\gamma$ uma curva de Jordan ( fechada e simples) regular $C^{1}$ por partes orientada positivamente no plano $\left(\mathbb{R}^{2}\right.$ ou $\left.\mathbb{C}\right), \Omega=\gamma \cup$ int $\gamma$ e $\omega \in C^{1}(\Omega) \cap C^{0}(\bar{\Omega})$. Então,

$$
\begin{gathered}
\iint_{\Omega} \frac{\partial \omega}{\partial \bar{z}}(x, y) d x d y=\frac{1}{2 i} \int_{\gamma} \omega(x, y) d z \\
\iint_{\Omega} \frac{\partial \omega}{\partial z}(x, y) d x d y=-\frac{1}{2 i} \int_{\gamma} \omega(x, y) d \bar{z}
\end{gathered}
$$

Demonstração.

Seja $\omega(x, y)=\mu(x, y)+i \nu(x, y)$. Vamos mostrar a igualdade $(1.7)$ :

$$
\begin{aligned}
\iint_{\Omega} \frac{\partial \omega}{\partial \bar{z}}(x, y) d x d y= & \frac{1}{2} \iint_{\Omega} \frac{\partial \omega}{\partial x}(x, y) d x d y+\frac{i}{2} \iint_{\Omega} \frac{\partial \omega}{\partial y}(x, y) d x d y \\
= & \frac{1}{2} \iint_{\Omega} \frac{\partial \mu}{\partial x}(x, y) d x d y+\frac{i}{2} \iint_{\Omega} \frac{\partial \nu}{\partial x}(x, y) d x d y+ \\
& +\frac{i}{2} \iint_{\Omega} \frac{\partial \mu}{\partial y}(x, y) d x d y-\frac{1}{2} \iint_{\Omega} \frac{\partial \nu}{\partial y}(x, y) d x d y
\end{aligned}
$$

Sabemos que $\mu, \nu \in C^{1}(\Omega ; \mathbb{R})$; assim, usando o teorema de Green no anterior, temos

$$
\begin{aligned}
\iint_{\Omega} \frac{\partial \omega}{\partial \bar{z}}(x, y) d x d y & =\frac{1}{2} \int_{\gamma} \mu(x, y) d y+\frac{i}{2} \int_{\gamma} \nu(x, y) d y-\frac{i}{2} \int_{\gamma} \mu(x, y) d x+\frac{1}{2} \int_{\gamma} \nu(x, y) d x \\
& =\frac{1}{2} \int_{\gamma} \omega(x, y) d y-\frac{i}{2} \int_{\gamma} \omega(x, y) d x \\
& =\frac{1}{2 i} \int_{\gamma} \omega(x, y) d z
\end{aligned}
$$

A demonstração da igualdade (1.8) é semelhante à anterior.

Observação 1.12. Nas hipóteses da proposição 1.2 e pelo fato que

$$
d z d \bar{z}=\frac{d(z, \bar{z})}{d(x, y)} d x d y=\left|\begin{array}{rr}
1 & 1 \\
i & -i
\end{array}\right| d x d y=-2 i d x d y
$$

temos que:

$$
\int_{\gamma} \omega(x, y) d z=-\iint_{\Omega} \frac{\partial \omega}{\partial \bar{z}}(x, y) d z d \bar{z}
$$


$e$

$$
\int_{\gamma} \omega(x, y) d \bar{z}=\iint_{\Omega} \frac{\partial \omega}{\partial z}(x, y) d z d \bar{z}
$$

Teorema 1.10 (Fórmula integral de Cauchy não homogênea). Seja $\gamma$ uma curva de Jordan regular $C^{1}$ por partes orientada positivamente no plano $\left(\mathbb{R}^{2}\right.$ ou $\left.\mathbb{C}\right)$, $\Omega=\gamma \cup$ int $\gamma$ e $\omega \in C^{1}(\bar{\Omega} ; \mathbb{C})$. Então,

$$
\omega(z)=\frac{1}{2 \pi i} \int_{\gamma} \frac{\omega(\zeta)}{\zeta-z} d \zeta-\frac{1}{\pi} \iint_{\Omega} \frac{\partial \omega}{\partial \bar{\zeta}}(\zeta) \cdot \frac{d \xi d \eta}{\zeta-z}
$$

$e$

$$
\omega(z)=-\frac{1}{2 \pi i} \int_{\gamma} \frac{\omega(\zeta)}{\bar{\zeta}-\bar{z}} d \bar{\zeta}-\frac{1}{\pi} \iint_{\Omega} \frac{\partial \omega}{\partial \zeta}(\zeta) \cdot \frac{d \xi d \eta}{\bar{\zeta}-\bar{z}}
$$

com $z \in \Omega, \zeta=\xi+i \eta$.

Demonstração.

Vamos mostrar a igualdade (1.9).

Seja $z_{0} \in \Omega$ um ponto fixado qualquer, $D_{R}=D\left(z_{0}, R\right)$ e $\Omega_{R}=\Omega \backslash D_{R}$, como podemos observar na figura 1.1.

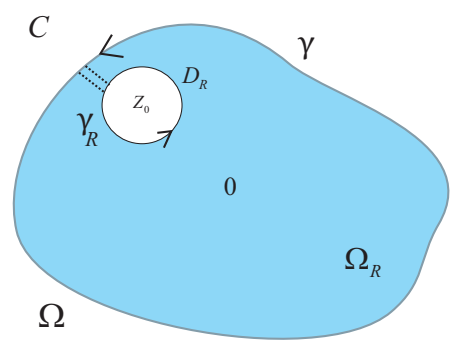

Figura 1.1

Como $\omega \in C^{1}(\bar{\Omega} ; \mathbb{C})$ e $\frac{1}{\zeta-z_{0}} \in C^{\infty}\left(\Omega_{R}\right), \operatorname{temos} \omega(\zeta) \cdot \frac{1}{\zeta-z_{0}} \in C^{1}\left(\Omega_{R}\right)$

Logo, pela proposição 1.2, temos que

$$
\begin{gathered}
\iint_{\Omega_{R}} \frac{\partial}{\partial \bar{\zeta}}\left(\omega(\zeta) \cdot \frac{1}{\zeta-z_{0}}\right) d \xi d \eta=\frac{1}{2 i} \int_{\gamma \cup\left\{-\gamma_{R}\right\}} \omega(\zeta) \cdot \frac{1}{\zeta-z_{0}} d \zeta \quad \Rightarrow \\
\iint_{\Omega_{R}} \frac{\partial \omega}{\partial \bar{\zeta}}(\zeta) \cdot \frac{d \xi d \eta}{\zeta-z_{0}}+\iint_{\Omega_{R}} \omega(\zeta) \cdot \frac{\partial}{\partial \bar{\zeta}}\left(\frac{1}{\zeta-z_{0}}\right) d \xi d \eta=\frac{1}{2 i} \int_{\gamma} \frac{\omega(\zeta)}{\zeta-z_{0}} d \zeta-\frac{1}{2 i} \int_{\gamma_{R}} \frac{\omega(\zeta)}{\zeta-z_{0}} d \zeta .
\end{gathered}
$$


Como $\frac{\partial}{\partial \bar{\zeta}}\left(\frac{1}{\zeta-z_{0}}\right)=0$ em $\Omega_{R}$, fazendo $R \rightarrow 0$ temos

$$
\frac{1}{2 i} \int_{\gamma} \frac{\omega(\zeta)}{\zeta-z_{0}} d \zeta-\frac{1}{2 i} \lim _{R \rightarrow 0} \underbrace{\int_{\gamma_{R}} \frac{\omega(\zeta)}{\zeta-z_{0}} d \zeta}_{(A)}=\lim _{R \rightarrow 0} \iint_{\Omega_{R}} \frac{\partial \omega}{\partial \bar{\zeta}}(\zeta) \cdot \frac{d \xi d \eta}{\zeta-z_{0}}
$$

$\operatorname{Em}(A)$ temos

$$
\int_{\gamma_{R}} \frac{\omega(\zeta)}{\zeta-z_{0}} d \zeta=\underbrace{\int_{\gamma_{R}} \frac{\omega(\zeta)-\omega\left(z_{0}\right)}{\zeta-z_{0}} d \zeta}_{(B)}+\underbrace{\int_{\gamma_{R}} \frac{\omega\left(z_{0}\right)}{\zeta-z_{0}} d \zeta}_{(C)}
$$

$\operatorname{Em}(B)$, como $\Omega$ é limitado e $\omega^{\prime}$ é contínua em $\bar{\Omega}$, temos que $\omega$ é Lipschitziana com constante de Lipschitz $K_{1}$, assim

$$
|B| \leq \int_{\gamma_{R}} \frac{\left|\omega(\zeta)-\omega\left(z_{0}\right)\right|}{\left|\zeta-z_{0}\right|}|d \zeta| \leq \int_{\gamma_{R}} K_{1}|d \zeta|=2 \pi R K_{1} .
$$

Em $(C)$, pela fórmula integral de Cauchy clássica, temos

$$
C=\int_{\gamma_{R}} \frac{\omega\left(z_{0}\right)}{\zeta-z_{0}} d \zeta=\omega\left(z_{0}\right) \int_{\gamma_{R}} \frac{d \zeta}{\zeta-z_{0}}=2 \pi i \omega\left(z_{0}\right) .
$$

Logo, por $(* 2)$ temos

$$
\lim _{R \rightarrow 0} \int_{\gamma_{R}} \frac{\omega(\zeta)}{\zeta-z_{0}} d \zeta=\lim _{R \rightarrow 0}(B+C)=2 \pi i \omega\left(z_{0}\right)
$$

Assim, por $(* 1)$ temos

$$
\frac{1}{2 i} \int_{\gamma} \frac{\omega(\zeta)}{\zeta-z_{0}} d \zeta-\frac{1}{2 i}\left(2 \pi i \omega\left(z_{0}\right)\right)=\iint_{\Omega} \frac{\partial \omega}{\partial \bar{\zeta}}(\zeta) \cdot \frac{d \xi d \eta}{\zeta-z_{0}}
$$

Finalmente,

$$
\omega\left(z_{0}\right)=\frac{1}{2 \pi i} \int_{\gamma} \frac{\omega(\zeta)}{\zeta-z_{0}} d \zeta-\frac{1}{\pi} \iint_{\Omega} \frac{\partial \omega}{\partial \bar{\zeta}}(\zeta) \cdot \frac{d \xi d \eta}{\zeta-z_{0}}
$$

Como $z_{0} \in \Omega$ foi escolhido arbitrário, temos que a igualdade anterior se cumpre para todo $z \in \Omega$.

De maneira análoga demonstra-se a igualdade (1.10). 
Resumindo: Seja $\gamma$ uma curva de Jordan regular $C^{1}$ por partes orientada positivamente no plano $\left(\mathbb{R}^{2}\right.$ ou $\left.\mathbb{C}\right)$ e $\Omega=\gamma \cup$ int $\gamma$. Se $\omega \in C^{1}(\bar{\Omega} ; \mathbb{C})$ é solução da equação $\frac{\partial \omega}{\partial \bar{z}}=f$ então

$$
\omega(z)=\Phi(z)-\frac{1}{\pi} \iint_{\Omega} \frac{f(\zeta)}{\zeta-z} d \xi d \eta
$$

sendo $\Phi(z)=\frac{1}{2 \pi i} \int_{\gamma} \frac{\omega(\zeta)}{\zeta-z} d \zeta \quad$ e $\quad \zeta=\xi+i \eta$.

A equação (1.11) chama-se Fórmula de Cauchy-Pompeiu.

\subsection{Funções Hölder contínuas}

Definição 1.23 (Função Hölder contínua). Seja $U \subset \mathbb{R}^{n}$ um aberto e $0<\alpha<1$. Dizemos que $\mu: U \rightarrow \mathbb{K}(\mathbb{R}$ ou $\mathbb{C})$ é uma função Hölder contínua em $U$ com exponente $\alpha$, se existe uma constante $c>0$ tal que

$$
|\mu(x)-\mu(y)| \leq c|x-y|^{\alpha}, \forall x, y \in U
$$

Observação 1.13. Sejam $U \subset \mathbb{R}^{n}$ um aberto limitado e $\mu: U \rightarrow \mathbb{K}(\mathbb{R}$ ou $\mathbb{C})$. Se $\mu$ é Hölder contínua em $U$ com expoente $\alpha$ então $\mu$ é Hölder contínua em $U$ com qualquer expoente $\beta \leq \alpha$.

Definição 1.24 (Seminorma e Norma de Hölder). Seja $\mu: U \rightarrow \mathbb{K}(\mathbb{R}$ ou $\mathbb{C}$ ) uma função contínua e limitada.

1. Definimos a seminorma de Hölder de $\mu$ por:

$$
[\mu]_{C^{0, \alpha}(\bar{U})}=\sup _{x \neq y}\left\{\frac{|\mu(x)-\mu(y)|}{|x-y|^{\alpha}}\right\} .
$$

2. Definimos a norma de Hölder de $\mu$ por:

$$
\|\mu\|_{C^{0, \alpha}(\bar{U})}=\|\mu\|_{C(\bar{U})}+[\mu]_{C^{0, \alpha}(\bar{U})}
$$

sendo $\|\mu\|_{C(\bar{U})}=\sup _{x \in U}\{|\mu(x)|\}$.

Definição 1.25 (O espaço de Hölder). O espaço de Hölder $C^{k, \alpha}(\bar{U})$, sendo $0<\alpha<1$, consiste de todas as funções $\mu \in C^{k}(\bar{U})$, para o qual a norma

$$
\|\mu\|_{C^{k, \alpha}(\bar{U})}=\sum_{|\beta| \leq k}\left\|D^{\beta} \mu\right\|_{C(\bar{U})}+\sum_{|\beta|=k}\left[D^{\beta} \mu\right]_{C^{0, \alpha}(\bar{U})}
$$


é finita. Isto é, o espaço de Hölder $C^{k, \alpha}(\bar{U})$ consiste das funções $\mu$ que são k-vezes continuamente diferenciáveis e cujas k-ésimas derivadas parciais são limitadas e Hölder contínuas com exponente $\alpha$.

Observações 1.1. Seja $\Omega \in \mathbb{C}$ um aberto, conexo e limitado. Então:

1. Se $f \in C^{\alpha}(\Omega)$, com $0<\alpha<1$ então $f$ é limitada em $\Omega$.

2. Se $f, g \in C^{\alpha}(\Omega)$, com $0<\alpha<1$ então $f+g \in C^{\alpha}(\Omega)$, com $0<\alpha<1$.

3. Se $f, g \in C^{\alpha}(\Omega)$, com $0<\alpha<1$ então f.g $\in C^{\alpha}(\Omega)$, com $0<\alpha<1$.

4. Se $f \in C^{1}(\Omega)$ e $f$ é limitada em $\Omega$ então $f \in C^{\alpha}(\Omega)$, para qualquer $0<\alpha<1$.

5. Se $f \in C^{\alpha}(\Omega), \Omega_{0}$ um subconjunto compacto de $\Omega, \varphi \in C_{c}^{\infty}(\Omega)$ e $\varphi \equiv 1$ em $\Omega_{0}$ então $\varphi \cdot f \in C^{\alpha}(\mathbb{C})$.

Teorema 1.11. O espaço $\left(C^{k, \alpha}(\bar{U}),\|\cdot\|_{C^{k, \alpha}(\bar{U})}\right)$ é um espaço de Banach.

A demonstração deste teorema pode ser encontrado em [10].

\subsubsection{Lemas sobre funções Hölder contínuas}

Lema 1.2. Seja $f \in C^{\alpha}$ em $D_{R}=\{z:|z|<R\}$. Então, a função $q: \mathbb{C} \rightarrow \mathbb{C}$ dada por

$$
q(z)=-\frac{1}{\pi} \iint_{D_{R}} \frac{f(\zeta)}{\zeta-z} d \xi d \eta
$$

tem derivadas parciais contínuas em $D_{R}$ dadas por

$$
\frac{\partial q}{\partial \bar{z}}(z)=f(z) \quad e \quad \frac{\partial q}{\partial z}(z)=-\frac{1}{\pi} \iint_{D_{R}} \frac{f(\zeta)-f(z)}{(\zeta-z)^{2}} d \xi d \eta
$$

A demonstração deste lema pode ser encontrado em [6].

Agora, faremos a prova do seguinte lema o qual tem hipótese mais forte que o lema anterior. 
Lema 1.3. Seja $f \in C_{c}^{1}$ em $D_{R}=\{z:|z|<R\}$. Então, a função $q: D_{R} \rightarrow \mathbb{C}$ dada por

$$
q(z)=-\frac{1}{\pi} \iint_{D_{R}} \frac{f(\zeta)}{\zeta-z} d \xi d \eta
$$

tem derivadas parciais continuas em $D_{R}$ dadas por

$$
\frac{\partial q}{\partial \bar{z}}(z)=f(z) \quad e \quad \frac{\partial q}{\partial z}(z)=-\frac{1}{\pi} \iint_{D_{R}} \frac{f(\zeta)-f(z)}{(\zeta-z)^{2}} d \xi d \eta
$$

Demonstração.

Seja $z \in D_{R}$ um ponto qualquer. Considere $\mu(z)=\frac{1}{z}$ e $\nu(z)=f(z)$.

Assim, sabemos que $\mu \in L^{1}\left(D_{R}\right)$ e $\nu \in C_{c}^{1}\left(D_{R}\right)$. Logo,

$$
\begin{aligned}
(\mu * \nu)(z) & =\iint_{D_{R}} \mu(z-\zeta) \cdot \nu(\zeta) d \xi d \eta \\
& =\iint_{D_{R}} \frac{1}{z-\zeta} \cdot f(\zeta) d \xi d \eta \\
& =-\iint_{D_{R}} \frac{1}{\zeta-z} \cdot f(\zeta) d \xi d \eta
\end{aligned}
$$

Daí, $q(z)=\frac{1}{\pi}(\mu * \nu)(z)$; logo, pelo lema 1.1 temos

$$
\begin{aligned}
& \frac{\partial q}{\partial x}(z)=\frac{1}{\pi}\left(\frac{\partial \mu}{\partial x} * \nu\right)(z)=\frac{1}{\pi}\left(\mu * \frac{\partial \nu}{\partial x}\right)(z) \\
& \frac{\partial q}{\partial y}(z)=\frac{1}{\pi}\left(\frac{\partial \mu}{\partial y} * \nu\right)(z)=\frac{1}{\pi}\left(\mu * \frac{\partial \nu}{\partial y}\right)(z)
\end{aligned}
$$

Logo,

$$
\frac{\partial q}{\partial x}(z)=-\frac{1}{\pi} \iint_{D_{R}} \frac{\partial f}{\partial \xi}(\zeta) \cdot \frac{1}{\zeta-z} d \xi d \eta \quad \text { e } \quad \frac{\partial q}{\partial y}(z)=-\frac{1}{\pi} \iint_{D_{R}} \frac{\partial f}{\partial \eta}(\zeta) \cdot \frac{1}{\zeta-z} d \xi d \eta .
$$

Assim,

$$
\frac{\partial q}{\partial \bar{z}}(z)=-\frac{1}{\pi} \iint_{D_{R}} \frac{\partial f}{\partial \bar{\zeta}}(\zeta) \cdot \frac{1}{\zeta-z} d \xi d \eta \quad \text { e } \quad \frac{\partial q}{\partial z}(z)=-\frac{1}{\pi} \iint_{D_{R}} \frac{\partial f}{\partial \zeta}(\zeta) \cdot \frac{1}{\zeta-z} d \xi d \eta
$$


Afirmação 1: $\frac{\partial q}{\partial \bar{z}}(z)=f(z), \forall z \in D_{R}$.

De fato, seja $\Omega_{\epsilon}=D_{R} \backslash D_{\epsilon}(z), \operatorname{com} \zeta=\xi+i \eta$ e $z=x+i y \in D_{R}$. Temos

$$
\begin{aligned}
& \frac{\partial q}{\partial \bar{z}}(z)=-\frac{1}{\pi} \iint_{D_{R}} \frac{\partial f}{\partial \bar{\zeta}}(\zeta) \cdot \frac{1}{\zeta-z} d \xi d \eta=-\frac{1}{\pi} \lim _{\epsilon \rightarrow 0} \iint_{\Omega_{\epsilon}} \frac{\partial f}{\partial \bar{\zeta}}(\zeta) \cdot \frac{1}{\zeta-z} d \xi d \eta \\
& =\frac{1}{2 \pi i} \lim _{\epsilon \rightarrow 0} \iint_{\Omega_{\epsilon}} \frac{\partial}{\partial \bar{\zeta}}\left(\frac{f(\zeta)}{\zeta-z}\right) d \zeta d \bar{\zeta} \\
& =-\frac{1}{2 \pi i} \lim _{\epsilon \rightarrow 0} \int_{\partial \Omega_{\epsilon}} \frac{f(\zeta)}{\zeta-z} d \zeta \quad \text { (por observação 1.12) } \\
& =-\frac{1}{2 \pi i} \underbrace{\int_{\gamma_{R}} \frac{f(\zeta)}{\zeta-z} d \zeta}_{=0}+\frac{1}{2 \pi i} \lim _{\epsilon \rightarrow 0} \int_{\gamma_{\epsilon}} \frac{f(\zeta)}{\zeta-z} d \zeta \\
& =\frac{1}{2 \pi i} \lim _{\epsilon \rightarrow 0} \underbrace{\int_{\gamma_{\epsilon}} \frac{f(\zeta)-f(z)}{\zeta-z} d \zeta}_{\left(I_{1}\right)}+\frac{1}{2 \pi i} \cdot f(z) \cdot \lim _{\epsilon \rightarrow 0} \underbrace{\int_{\gamma_{\epsilon}} \frac{1}{\zeta-z} d \zeta}_{\left(I_{2}\right)} .
\end{aligned}
$$

Agora, como $\left|I_{1}\right| \leq \int_{\gamma_{\epsilon}} \frac{|f(\zeta)-f(z)|}{|\zeta-z|}|d \zeta| \leq K_{1} \int_{\gamma_{\epsilon}}|d \zeta|=2 \pi \epsilon K_{1}$, sendo $K_{1}=\sup _{z \in D_{R}}\left\{\left|f^{\prime}(z)\right|\right\}$.

Temos que, $\lim _{\epsilon \rightarrow 0} I_{1}=0$. Pela fórmula integral de Cauchy clássica, temos $I_{2}=2 \pi i$.

Portanto,

$$
\frac{\partial q}{\partial \bar{z}}(z)=\frac{1}{2 \pi i} \cdot 0+\frac{1}{2 \pi i} f(z) \cdot(2 \pi i)=f(z)
$$

Afirmação 2: $\frac{\partial q}{\partial z}(z)=-\frac{1}{\pi} \iint_{D_{R}} \frac{f(\zeta)-f(z)}{(\zeta-z)^{2}} d \xi d \eta$.

De fato, sabemos que

$$
\begin{aligned}
\frac{\partial}{\partial \zeta}\left(\frac{f(\zeta)-f(z)}{\zeta-z}\right) & =\frac{\partial}{\partial \zeta}(f(\zeta)-f(z)) \cdot \frac{1}{\zeta-z}+(f(\zeta)-f(z)) \cdot \frac{\partial}{\partial \zeta}\left(\frac{1}{\zeta-z}\right) \\
& =\frac{\partial f}{\partial \zeta}(\zeta) \cdot \frac{1}{\zeta-z}-\frac{f(\zeta)-f(z)}{(\zeta-z)^{2}} \Rightarrow
\end{aligned}
$$




$$
\frac{\partial f}{\partial \zeta}(\zeta) \cdot \frac{1}{\zeta-z}=\frac{\partial}{\partial \zeta}\left(\frac{f(\zeta)-f(z)}{\zeta-z}\right)+\frac{f(\zeta)-f(z)}{(\zeta-z)^{2}}
$$

Assim, seja $\Omega_{\epsilon}=D_{R} \backslash D_{\epsilon}(z), \operatorname{com} \zeta=\xi+i \eta$ e $z=x+i y \in D_{R}$. Temos

$$
\begin{aligned}
& \frac{\partial q}{\partial z}(z)=-\frac{1}{\pi} \iint_{D_{R}} \frac{\partial f}{\partial \zeta}(\zeta) \cdot \frac{1}{\zeta-z} d \xi d \eta \\
& =-\frac{1}{\pi} \iint_{D_{R}} \frac{\partial}{\partial \zeta}\left(\frac{f(\zeta)-f(z)}{\zeta-z}\right) d \xi d \eta-\frac{1}{\pi} \iint_{D_{R}} \frac{f(\zeta)-f(z)}{(\zeta-z)^{2}} d \xi d \eta \quad(\operatorname{por}(* 1)) \\
& =\frac{1}{2 \pi i} \lim _{\epsilon \rightarrow 0} \iint_{\Omega_{\epsilon}} \frac{\partial}{\partial \zeta}\left(\frac{f(\zeta)-f(z)}{\zeta-z}\right) d \zeta d \bar{\zeta}-\frac{1}{\pi} \iint_{D_{R}} \frac{f(\zeta)-f(z)}{(\zeta-z)^{2}} d \xi d \eta, \\
& =\frac{1}{2 \pi i} \lim _{\epsilon \rightarrow 0} \int_{\partial \Omega_{\epsilon}} \frac{f(\zeta)-f(z)}{\zeta-z} d \bar{\zeta}-\frac{1}{\pi} \iint_{D_{R}} \frac{f(\zeta)-f(z)}{(\zeta-z)^{2}} d \xi d \eta \quad \text { (por observação 1.12) } \\
& =\frac{1}{2 \pi i} \int_{\gamma_{R}} \frac{f(\zeta)-f(z)}{\zeta-z} d \bar{\zeta}-\frac{1}{2 \pi i} \lim _{\epsilon \rightarrow 0} \int_{\gamma_{\epsilon}} \frac{f(\zeta)-f(z)}{\zeta-z} d \bar{\zeta}-\frac{1}{\pi} \iint_{D_{R}} \frac{f(\zeta)-f(z)}{(\zeta-z)^{2}} d \xi d \eta \\
& =\frac{1}{2 \pi i} \underbrace{\int_{0}^{2 \pi} \frac{f\left(\gamma_{R}(t)\right)-f(z)}{\gamma_{R}(t)-z}\left(-i R e^{-i t}\right) d t}_{I_{3}}-\frac{1}{2 \pi i} \lim _{\epsilon \rightarrow 0} \underbrace{\int_{\gamma_{\epsilon}} \frac{f(\zeta)-f(z)}{\zeta-z} d \bar{\zeta}}_{I_{4}}+ \\
& -\frac{1}{\pi} \iint_{D_{R}} \frac{f(\zeta)-f(z)}{(\zeta-z)^{2}} d \xi d \eta
\end{aligned}
$$

Agora, temos que

$\left|I_{3}\right| \leq \int_{0}^{2 \pi} \frac{\left|f\left(\gamma_{R}(t)\right)-f(z)\right|}{\left|\gamma_{R}(t)-z\right|}\left|R e^{-i t}\right| d t \leq \delta \cdot R \int_{0}^{2 \pi} \frac{d t}{R-|z|}=\frac{2 \pi \delta R}{R-|z|} \rightarrow 0$ quando $\delta \rightarrow 0$

$\mathrm{e}\left|I_{4}\right| \leq \int_{\gamma_{\epsilon}} \frac{|f(\zeta)-f(z)|}{|\zeta-z|}|d \bar{\zeta}| \leq K_{1} \int_{\gamma_{\epsilon}}|d \bar{\zeta}|=2 \pi \epsilon K_{1} \rightarrow 0$ quando $\epsilon \rightarrow 0$, sendo

$K_{1}=\sup _{z \in D_{R}}\left|f^{\prime}(z)\right|$

Portanto,

$$
\frac{\partial q}{\partial z}(z)=-\frac{1}{\pi} \iint_{D_{R}} \frac{f(\zeta)-f(z)}{(\zeta-z)^{2}} d \xi d \eta .
$$


Lema 1.4. Seja $f \in C^{\alpha}$ com coeficiente $H$ em $D_{r}=\{z:|z|<r\}$. Seja $p: D_{r} \rightarrow \mathbb{C}$ uma função dada por

$$
p(z)=-\frac{1}{\pi} \iint_{D_{r}} \frac{f(\zeta)-f(z)}{(\zeta-z)^{2}} d \xi d \eta,
$$

$\operatorname{com} \zeta=\xi+i \eta$. Então, existe uma constante $C$ que depende unicamente de a tal que

$$
|p(z)| \leq C H r^{\alpha}
$$

e p é Hölder contínua com exponente $\alpha$ e coeficiente $C H$.

Demonstração.

Primeiro mostremos que existe $C_{1}$ que depende unicamente de $\alpha$ tal que $|p(z)| \leq C_{1} H r^{\alpha}$. Seja z um ponto fixado em $D_{r}$. Assim,

$$
\begin{aligned}
|p(z)| & \leq \frac{1}{\pi} \iint_{D_{r}} \frac{|f(\zeta)-f(z)|}{|\zeta-z|^{2}} d \xi d \eta \leq \frac{H}{\pi} \iint_{D_{r}} \frac{d \xi d \eta}{|\zeta-z|^{2-\alpha}} \\
& \leq \frac{H}{\pi} \iint_{|\zeta-z|<2 r} \frac{d \xi d \eta}{|\zeta-z|^{2-\alpha}} \leq \frac{H}{\pi} \int_{0}^{2 \pi} \int_{0}^{2 r} \frac{1}{r_{1}^{2-\alpha}} \cdot r_{1} d r_{1} d \theta \\
& \leq \frac{H}{\pi} \int_{0}^{2 \pi} \int_{0}^{2 r} r_{1}^{\alpha-1} d r_{1} d \theta=\frac{2^{\alpha+1}}{\alpha} \cdot H \cdot r^{\alpha} \\
& \leq C_{1} H r^{\alpha},
\end{aligned}
$$

sendo $C_{1}=\frac{2^{\alpha+1}}{\alpha}$

Agora, mostremos que $p(z)$ é Hölder contínua. Para isto, consideremos $z_{1}$ e $z_{3}$ pontos fixados em $D_{r}$ tais que $z_{1} \neq z_{3}$ e $\delta=\left|z_{1}-z_{3}\right|$.

Seja $z_{2}$ um ponto em $D_{r}$ que satisfaz

$$
\left|z_{2}-z_{1}\right| \leq \delta, \quad\left|z_{2}-z_{3}\right| \leq \delta \quad \text { e } \quad r-\left|z_{2}\right| \geq \frac{\delta}{10}
$$

Por exemplo, veja a figura 1.2. 


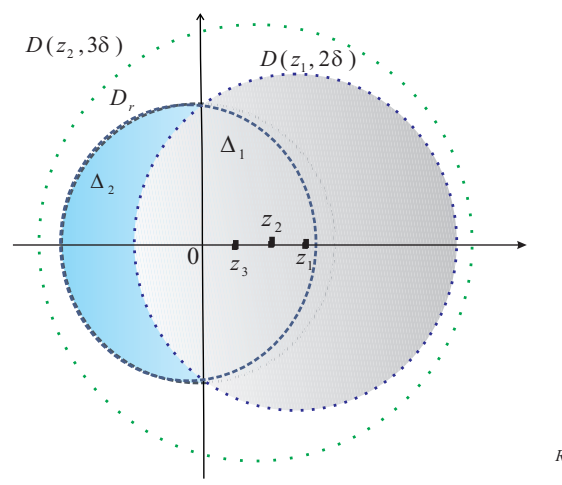

Figura 1.2

Pela desigualdade triangular temos

$$
\left|p\left(z_{1}\right)-p\left(z_{3}\right)\right| \leq \underbrace{\left|p\left(z_{1}\right)-p\left(z_{2}\right)\right|}_{(I)}+\underbrace{\left|p\left(z_{2}\right)-p\left(z_{3}\right)\right|}_{(I I)} .
$$

- Para $(I)$ temos

$$
p\left(z_{1}\right)-p\left(z_{2}\right)=-\frac{1}{\pi} \iint_{D_{r}} g(\zeta) d \xi d \eta
$$

sendo

$$
g(\zeta)=\frac{f(\zeta)-f\left(z_{1}\right)}{\left(\zeta-z_{1}\right)^{2}}-\frac{f(\zeta)-f\left(z_{2}\right)}{\left(\zeta-z_{2}\right)^{2}}
$$

Denotemos: $\Delta_{1}=D_{r} \cap D\left(z_{1}, 2 \delta\right) \quad$ e $\quad \Delta_{2}=D_{r} \backslash \Delta_{1}$. Note que, se $\delta>r$ então $D_{r} \subset D\left(z_{1}, 2 \delta\right)$. Consequentemente, teríamos que $\Delta_{2}=\emptyset$.

Portanto, para nossas contas assumiremos que $\delta \leq r$. Assim

$$
\left|p\left(z_{1}\right)-p\left(z_{2}\right)\right| \leq \frac{1}{\pi} \iint_{D_{r}}|g(\zeta)| d \xi d \eta=\frac{1}{\pi} \iint_{\Delta_{1}}|g(\zeta)| d \xi d \eta+\frac{1}{\pi} \iint_{\Delta_{2}}|g(\zeta)| d \xi d \eta
$$

1. Estimemos a integral de $g$ sobre $\Delta_{1}$ :

$$
\begin{aligned}
\frac{1}{\pi} \iint_{\Delta_{1}}|g(\zeta)| d \xi d \eta & \leq \frac{1}{\pi} \iint_{\Delta_{1}} \frac{\left|f(\zeta)-f\left(z_{1}\right)\right|}{\left|\zeta-z_{1}\right|^{2}} d \xi d \eta+\frac{1}{\pi} \iint_{\Delta_{1}} \frac{\left|f(\zeta)-f\left(z_{2}\right)\right|}{\left|\zeta-z_{2}\right|^{2}} d \xi d \eta \\
& \leq \frac{H}{\pi} \iint_{\Delta_{1}} \frac{d \xi d \eta}{\left|\zeta-z_{1}\right|^{2-\alpha}}+\frac{H}{\pi} \iint_{\Delta_{1}} \frac{d \xi d \eta}{\left|\zeta-z_{2}\right|^{2-\alpha}}
\end{aligned}
$$

Como $\Delta_{1} \subset D\left(z_{1}, 2 \delta\right)$ e $\Delta_{1} \subset D\left(z_{2}, 3 \delta\right)$, temos o seguinte 


$$
\frac{1}{\pi} \iint_{\Delta_{1}}|g(\zeta)| d \xi d \eta \leq \frac{H}{\pi} \underbrace{\iint_{\left|\zeta-z_{1}\right|<2 \delta} \frac{d \xi d \eta}{\left|\zeta-z_{1}\right|^{2-\alpha}}}_{(I I I)}+\frac{H}{\pi} \underbrace{\iint_{\left|\zeta-z_{2}\right|<3 \delta} \frac{d \xi d \eta}{\left|\zeta-z_{2}\right|^{2-\alpha}}}_{(I V)} .
$$

Para resolver $(I I I)$ e $(I V)$, usamos coordenadas polares. Assim, temos

$$
\begin{aligned}
\frac{1}{\pi} \iint_{\Delta_{1}}|g(\zeta)| d \xi d \eta & \leq \frac{H}{\pi} \int_{0}^{2 \pi} \int_{0}^{2 \delta} \frac{r_{1}}{r_{1}^{2-\alpha}} d r_{1} d \theta_{1}+\frac{H}{\pi} \int_{0}^{2 \pi} \int_{0}^{3 \delta} \frac{r_{2}}{r_{2}^{2-\alpha}} d r_{2} d \theta_{2} \\
& \leq \frac{H}{\pi} \int_{0}^{2 \pi} \int_{0}^{2 \delta} r_{1}^{\alpha-1} d r_{1} d \theta_{1}+\frac{H}{\pi} \int_{0}^{2 \pi} \int_{0}^{3 \delta} r_{2}^{\alpha-1} d r_{2} d \theta_{2} \\
& \leq \frac{H}{\pi} \cdot \frac{(2 \pi)(2 \delta)^{\alpha}}{\alpha}+\frac{H}{\pi} \cdot \frac{(2 \pi)(3 \delta)^{\alpha}}{\alpha} \\
& \leq \frac{2\left(2^{\alpha}+3^{\alpha}\right) H \delta^{\alpha}}{\alpha}=C_{2} \cdot H \delta^{\alpha}
\end{aligned}
$$

sendo $C_{2}=\frac{2\left(2^{\alpha}+3^{\alpha}\right)}{\alpha}$.

2. Estimemos a integral de $g$ sobre $\Delta_{2}$ :

Primeiro, temos

$$
\begin{aligned}
g(\zeta) & =\frac{f(\zeta)-f\left(z_{1}\right)}{\left(\zeta-z_{1}\right)^{2}}-\frac{f(\zeta)-f\left(z_{2}\right)}{\left(\zeta-z_{2}\right)^{2}}+\frac{f\left(z_{1}\right)}{\left(\zeta-z_{2}\right)^{2}}-\frac{f\left(z_{1}\right)}{\left(\zeta-z_{2}\right)^{2}} \\
& =\frac{f\left(z_{2}\right)-f\left(z_{1}\right)}{\left(\zeta-z_{2}\right)^{2}}+\left(f(\zeta)-f\left(z_{1}\right)\right) \cdot\left[\frac{1}{\left(\zeta-z_{1}\right)^{2}}-\frac{1}{\left(\zeta-z_{2}\right)^{2}}\right] \\
& =\frac{f\left(z_{2}\right)-f\left(z_{1}\right)}{\left(\zeta-z_{2}\right)^{2}}+\left(f(\zeta)-f\left(z_{1}\right)\right)\left(z_{2}-z_{1}\right) \cdot\left[\frac{z_{2}+z_{1}-2 \zeta}{\left(\zeta-z_{1}\right)^{2}\left(\zeta-z_{2}\right)^{2}}\right] \\
& =\frac{f\left(z_{2}\right)-f\left(z_{1}\right)}{\left(\zeta-z_{2}\right)^{2}}+\left(f(\zeta)-f\left(z_{1}\right)\right)\left(z_{2}-z_{1}\right)\left[\frac{-1}{\left(\zeta-z_{1}\right)^{2}\left(\zeta-z_{2}\right)}-\frac{1}{\left(\zeta-z_{1}\right)\left(\zeta-z_{2}\right)^{2}}\right] \\
& =\frac{f\left(z_{2}\right)-f\left(z_{1}\right)}{\left(\zeta-z_{2}\right)^{2}}+\left[\frac { ( f ( \zeta ) - f ( z _ { 1 } ) ) ( z _ { 1 } - z _ { 2 } ) } { ( \zeta - z _ { 1 } ) ^ { 3 } } \left[\frac{\left(\zeta-z_{2}\right.}{\left.\zeta-\frac{\left(\zeta-z_{1}\right)^{2}}{\left(\zeta-z_{2}\right)^{2}}\right]}\right.\right. \\
& =\underbrace{\frac{f\left(z_{2}\right)-f\left(z_{1}\right)}{\left(\zeta-z_{2}\right)^{2}}}_{=g_{1}(\zeta)}+\underbrace{\frac{\left(f(\zeta)-f\left(z_{1}\right)\right)\left(z_{1}-z_{2}\right)}{\left(\zeta-z_{1}\right)^{3}} \cdot \frac{\left(\zeta-z_{1}\right)}{\left(\zeta-z_{2}\right)}\left[1+\frac{\zeta-z_{1}}{\zeta-z_{2}}\right]}_{=g_{2}(\zeta)} .
\end{aligned}
$$


Assim,

$$
\frac{1}{\pi} \iint_{\Delta_{2}} g(\zeta) d \xi d \eta=\underbrace{\frac{1}{\pi} \iint_{\Delta_{2}} g_{1}(\zeta) d \xi d \eta}_{(V)}+\underbrace{\frac{1}{\pi} \iint_{\Delta_{2}} g_{2}(\zeta) d \xi d \eta}_{(V I)} .
$$

$\operatorname{Em}(V)$, temos

$$
\begin{aligned}
\frac{1}{\pi} \iint_{\Delta_{2}} g_{1}(\zeta) d \xi d \eta & =\frac{f\left(z_{2}\right)-f\left(z_{1}\right)}{\pi} \iint_{\Delta_{2}} \frac{d \xi d \eta}{\left(\zeta-z_{2}\right)^{2}} \\
& =\frac{f\left(z_{2}\right)-f\left(z_{1}\right)}{\pi}\left[\iint_{D_{r}} \frac{d \xi d \eta}{\left(\zeta-z_{2}\right)^{2}}-\iint_{\Delta_{1}} \frac{d \xi d \eta}{\left(\zeta-z_{2}\right)^{2}}\right] \\
& \leq \frac{f\left(z_{2}\right)-f\left(z_{1}\right)}{\pi}\left[\iint_{\left|\zeta-z_{2}\right| \leq 2 r} \frac{d \xi d \eta}{\left(\zeta-z_{2}\right)^{2}}-\iint_{\Delta_{1}} \frac{d \xi d \eta}{\left(\zeta-z_{2}\right)^{2}}\right] \\
& \leq \frac{f\left(z_{2}\right)-f\left(z_{1}\right)}{\pi}\left[\frac{1}{2 i} \int_{\left|\zeta-z_{2}\right|=2 r} \frac{d \bar{\zeta}}{\zeta-z_{2}}+\frac{1}{2 i} \int_{\partial \Delta_{1}} \frac{-d \bar{\zeta}}{\zeta-z_{2}}\right](\text { por prop.1.2) } \\
& \leq \frac{f\left(z_{1}\right)-f\left(z_{2}\right)}{\pi}\left[\frac{-1}{2 i} \int_{0}^{2 \pi} \frac{-2 i r e^{-i t}}{2 r e^{i t}} d t+\frac{1}{2 i} \int_{\partial \Delta_{1}} \frac{d \bar{\zeta}}{\zeta-z_{2}}\right] \\
& \leq \frac{f\left(z_{1}\right)-f\left(z_{2}\right)}{\pi}\left[0+\frac{1}{2 i} \int_{\partial \Delta_{1}} \frac{d \bar{\zeta}}{\zeta-z_{2}}\right]=\frac{f\left(z_{1}\right)-f\left(z_{2}\right)}{2 \pi i} \int_{\partial \Delta_{1}} \frac{d \bar{\zeta}}{\zeta-z_{2}}
\end{aligned}
$$

Daí,

$$
\frac{1}{\pi} \iint_{\Delta_{2}}\left|g_{1}(\zeta)\right| d \xi d \eta \leq \frac{\left|f\left(z_{1}\right)-f\left(z_{2}\right)\right|}{2 \pi} \int_{\partial \Delta_{1}} \frac{|d \bar{\zeta}|}{\left|\zeta-z_{2}\right|}
$$

Notemos que se $\zeta \in \Delta_{1}$, temos os casos:

$\circ$ Se $|\zeta|=r$ temos $\left|\zeta-z_{2}\right| \geq|\zeta|-\left|z_{2}\right|=r-\left|z_{2}\right| \geq \frac{\delta}{10} ;$

$\circ$ Se $\left|\zeta-z_{1}\right|=2 \delta$ temos $\left|\zeta-z_{2}\right| \geq\left|\zeta-z_{1}\right|-\left|z_{1}-z_{2}\right| \geq 2 \delta-\delta=\delta \geq \frac{\delta}{10}$.

Assim,

$$
\begin{aligned}
\frac{1}{\pi} \iint_{\Delta_{2}}\left|g_{1}(\zeta)\right| d \xi d \eta & \leq \frac{H\left|z_{1}-z_{2}\right|^{\alpha}}{2 \pi} \int_{\partial \Delta_{1}} \frac{10|d \bar{\zeta}|}{\delta} \leq \frac{5 H \delta^{\alpha-1}}{\pi} \int_{\partial D\left(z_{1}, 2 \delta\right)}|d \bar{\zeta}| \\
& \leq \frac{5 H \delta^{\alpha-1}}{\pi}(2 \pi)(2 \delta)=20 H \delta^{\alpha}=C_{3} H \delta^{\alpha}
\end{aligned}
$$


sendo $C_{3}=20$.

Em $(V I)$, notemos que se $\zeta \in \Delta_{2}$ acontece o seguinte:

Se $\left|\zeta-z_{1}\right| \geq 2 \delta$ e $\left|\zeta-z_{1}\right|<2 r$ temos que $2 \delta \leq\left|\zeta-z_{1}\right| \leq\left|\zeta-z_{2}\right|+\left|z_{2}-z_{1}\right| \leq\left|\zeta-z_{2}\right|+\delta$; $\log \mathrm{O}, \delta \leq\left|\zeta-z_{2}\right|$, assim $\frac{1}{\left|\zeta-z_{2}\right|} \leq \frac{1}{\delta}$.

Finalmente, $\left|\frac{\zeta-z_{1}}{\zeta-z_{2}}\right| \leq \frac{\left|\zeta-z_{2}\right|}{\left|\zeta-z_{2}\right|}+\frac{\left|z_{2}-z_{1}\right|}{\left|\zeta-z_{2}\right|} \leq 1+\frac{\delta}{\delta}=2$

Portanto,

$$
\begin{aligned}
\frac{1}{\pi} \iint_{\Delta_{2}}\left|g_{2}(\zeta)\right| d \xi d \eta & \leq \frac{1}{\pi} \iint_{\Delta_{2}} \frac{\left|f(\zeta)-f\left(z_{1}\right)\right|\left|z_{1}-z_{2}\right|}{\left|\zeta-z_{1}\right|^{3}} \cdot\left|\frac{\zeta-z_{1}}{\zeta-z_{2}}\right|\left(1+\left|\frac{\zeta-z_{1}}{\zeta-z_{2}}\right|\right) d \xi d \eta \\
& \leq \frac{H}{\pi} \iint_{\Delta_{2}} \frac{2 \delta(1+2)}{\left|\zeta-z_{1}\right|^{3-\alpha}} d \xi d \eta \\
& \leq \frac{6 H \delta}{\pi} \iint_{\Delta_{2}} \frac{d \xi d \eta}{\left|\zeta-z_{1}\right|^{3-\alpha}} \\
& \leq \frac{6 H \delta}{\pi} \iint_{\left|\zeta-z_{1}\right|>2 \delta} \frac{d \xi d \eta}{\left|\zeta-z_{1}\right|^{3-\alpha}} .
\end{aligned}
$$

Assim, usando coordenadas polares, temos que

$$
\begin{aligned}
\frac{1}{\pi} \iint_{\Delta_{2}}\left|g_{2}(\zeta)\right| d \xi d \eta & \leq \frac{6 H \delta}{\pi} \int_{0}^{2 \pi} \int_{2 \delta}^{\infty} \frac{r_{1}}{r_{1}^{3-\alpha}} d r_{1} d \theta=\frac{6 H \delta}{\pi} \int_{0}^{2 \pi} \int_{2 \delta}^{\infty} r_{1}^{\alpha-2} d r_{1} d \theta \\
& \leq \frac{6 H \delta}{\pi} \cdot \frac{2 \pi}{\alpha-1}\left[0-(2 \delta)^{\alpha-1}\right]=\frac{3 \cdot 2^{\alpha+1}}{1-\alpha} H \delta^{\alpha} \\
& \leq C_{4} H \delta^{\alpha}
\end{aligned}
$$

sendo $C_{4}=\frac{2^{\alpha+1} \cdot 3}{1-\alpha}$.

Logo, substituindo $(* 4),(* 5),(* 6)$ em $(* 3)$ temos

$$
\begin{aligned}
\left|p\left(z_{1}\right)-p\left(z_{2}\right)\right| & \leq C_{2} H \delta^{\alpha}+C_{3} H \delta^{\alpha}+C_{4} H \delta^{\alpha}=C_{5} H \delta^{\alpha} \\
& \leq C_{5} H\left|z_{1}-z_{3}\right|^{\alpha},
\end{aligned}
$$


sendo $C_{5}=C_{2}+C_{3}+C_{4}$.

- Para (II), trabalha-se analogamente ao caso $(I)$. Assim, obtemos

$$
\left|p\left(z_{2}\right)-p\left(z_{3}\right)\right| \leq C_{6} H\left|z_{1}-z_{3}\right|^{\alpha}
$$

Assim, em $(* 2)$ temos

$$
\left|p\left(z_{1}\right)-p\left(z_{3}\right)\right| \leq C_{5} H\left|z_{1}-z_{3}\right|^{\alpha}+C_{6} H\left|z_{1}-z_{3}\right|^{\alpha}=C_{7} H\left|z_{1}-z_{3}\right|^{\alpha},
$$

sendo $C_{7}=C_{5}+C_{6}$. Finalmente, tomemos $C=\max \left\{C_{1}, C_{7}\right\}$.

Portanto,

$$
\left|p\left(z_{1}\right)-p\left(z_{3}\right)\right| \leq C H\left|z_{1}-z_{3}\right|^{\alpha} .
$$

\subsection{Operador integral}

Definamos o operador integral $P$ agindo sobre funções $h \in L^{p}(\mathbb{C}), \operatorname{com} p>2$. Defina, Ph $: \mathbb{C} \rightarrow \mathbb{C}$, sendo

$$
\operatorname{Ph}(z)=-\frac{1}{\pi} \iint_{\mathbb{R}^{2}} h(\zeta) \cdot\left(\frac{1}{\zeta-z}-\frac{1}{\zeta}\right) d \xi d \eta,
$$

$\operatorname{com} \zeta=\xi+i \eta$

Lema 1.5. Seja Ph: $\mathbb{C} \rightarrow \mathbb{C}$ a função definida em (1.15), com $h \in L^{p}(\mathbb{C}), p>2$. Então, Ph é contínua e satisfaz a condição de ser Hölder contínua com expoente $1-2 / p$.

Demonstração.

Seja $h \in L^{p}(\mathbb{C})$, com $p>2$. Podemos escrever

$$
\operatorname{Ph}(z)=-\frac{1}{\pi} \iint_{\mathbb{R}^{2}} h(\zeta) \cdot \frac{z}{\zeta(\zeta-z)} d \xi d \eta
$$

para todo $z \in \mathbb{C}$.

Note que se $\frac{1}{p}+\frac{1}{q}=1$; então $2<p=\frac{q}{q-1}$. Daí, $1<q<2$.

Afirmação 1: $\frac{z}{\zeta(\zeta-z)} \in L^{q}(\mathbb{C})$, com $1<q<2$.

De fato, para $z=0$ o resultado é trivial. Vamos, então, considerar $z \neq 0$. 
Note que para $z \neq 0$ temos

$$
\iint_{\mathbb{R}^{2}} \frac{z}{\zeta(\zeta-z)} d \xi d \eta=\iint_{\mathbb{R}^{2}} \frac{d \xi d \eta}{\frac{\zeta}{z}\left(\frac{\zeta}{z}-1\right) z}=\frac{1}{z} \iint_{\mathbb{R}^{2}} \frac{d \xi d \eta}{\frac{\zeta}{z}\left(\frac{\zeta}{z}-1\right)}
$$

Agora, denotamos $z=x+i y$ e fazemos $\xi^{\prime}+i \eta^{\prime}=\zeta^{\prime}=\frac{\zeta}{z} ; \operatorname{logo}$, temos que $\xi^{\prime}+i \eta^{\prime}=\frac{x}{|z|^{2}} \cdot \xi+\frac{y}{|z|^{2}} \cdot \eta+i\left(\frac{x}{|z|^{2}} \cdot \eta-\frac{y}{|z|^{2}} \cdot \xi\right)$, isto é, $\xi^{\prime}=\frac{x}{|z|^{2}} \cdot \xi+\frac{y}{|z|^{2}} \cdot \eta \mathrm{e}$ $\eta^{\prime}=\frac{x}{|z|^{2}} \cdot \eta-\frac{y}{|z|^{2}} \cdot \xi$.

Daí,

$$
d \xi^{\prime} d \eta^{\prime}=\left|\begin{array}{cc}
\frac{\partial \xi^{\prime}}{\partial \xi} & \frac{\partial \eta^{\prime}}{\partial \xi} \\
\frac{\partial \xi^{\prime}}{\partial \eta} & \frac{\partial \eta^{\prime}}{\partial \eta}
\end{array}\right| d \xi d \eta=\left|\begin{array}{cc}
\frac{x}{|z|^{2}} & -\frac{y}{|z|^{2}} \\
\frac{y}{|z|^{2}} & \frac{x}{|z|^{2}}
\end{array}\right| d \xi d \eta=\frac{1}{|z|^{2}} d \xi d \eta
$$

Assim, em $(* 2)$ teríamos

$$
\iint_{\mathbb{R}^{2}} \frac{z}{\zeta(\zeta-z)} d \xi d \eta=\frac{|z|^{2}}{z} \iint_{\mathbb{R}^{2}} \frac{1}{\zeta^{\prime}\left(\zeta^{\prime}-1\right)} d \xi^{\prime} d \eta^{\prime}
$$

Portanto, basta verificar que $\frac{1}{\zeta(\zeta-1)} \in L^{q}(\mathbb{C})$, com $1<q<2$.

Para isso, defina as seguintes regiões:

$D_{1}=B(0,1 / 2), D_{2}=B(1,1 / 2), A_{1}=\left\{(x, y) \in \mathbb{R}^{2}: x \leq 1 / 2\right\} \backslash D_{1} \mathrm{e}$

$A_{2}=\left\{(x, y) \in \mathbb{R}^{2}: x>1 / 2\right\} \backslash D_{2}$. Assim, $\mathbb{R}^{2}=D_{1} \cup D_{2} \cup A_{1} \cup A_{2}$, como podemos observar na figura 1.3.

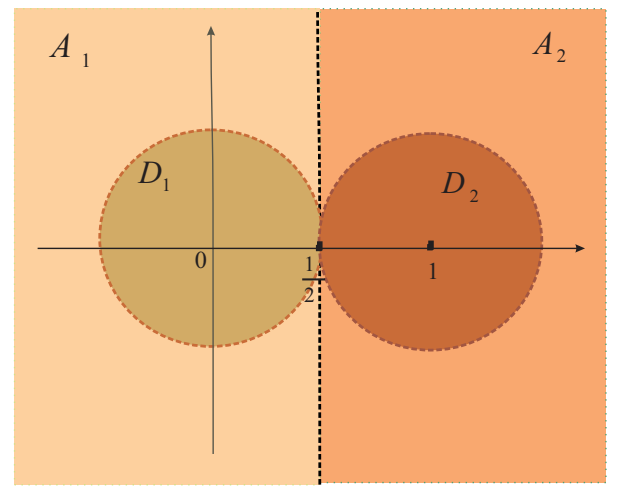

Figura 1.3 
Daí,

$$
\begin{aligned}
\iint_{\mathbb{R}^{2}} \frac{d \xi d \eta}{|\zeta|^{q}|\zeta-1|^{q}} & =\iint_{D_{1}} \frac{d \xi d \eta}{|\zeta|^{q}|\zeta-1|^{q}}+\iint_{D_{2}} \frac{d \xi d \eta}{|\zeta|^{q}|\zeta-1|^{q}}+ \\
& +\iint_{A_{1}} \frac{d \xi d \eta}{|\zeta|^{q}|\zeta-1|^{q}}+\iint_{A_{2}} \frac{d \xi d \eta}{|\zeta|^{q}|\zeta-1|^{q}}
\end{aligned}
$$

1. Integrando em $D_{1}$. Nesta região temos que $\frac{1}{2} \leq|\zeta-1|$; logo, $\frac{1}{|\zeta-1|} \leq 2$. Assim,

$$
\begin{aligned}
\iint_{D_{1}} \frac{d \xi d \eta}{|\zeta|^{q}|\zeta-1|^{q}} & \leq \iint_{D_{1}} \frac{2^{q}}{|\zeta|^{q}} d \xi d \eta \leq 2^{q} \int_{0}^{2 \pi} \int_{0}^{1 / 2} \frac{r}{r^{q}} d r d \theta \\
& \leq 2^{q}(2 \pi) \int_{0}^{1 / 2} r^{1-q} d r=\frac{2^{2 q-1} \pi}{2-q}
\end{aligned}
$$

2. Integrando em $D_{2}$. Nesta região temos que $\frac{1}{2} \leq|\zeta| \leq \frac{3}{2} ; \operatorname{logo}, \frac{1}{|\zeta|} \leq 2$. Assim,

$$
\begin{aligned}
\iint_{D_{2}} \frac{d \xi d \eta}{|\zeta|^{q}|\zeta-1|^{q}} & \leq \iint_{D_{2}} \frac{2^{q}}{|\zeta-1|^{q}} d \xi d \eta \leq 2^{q} \int_{0}^{2 \pi} \int_{0}^{1 / 2} \frac{r}{r^{q}} d r d \theta \\
& \leq 2^{q}(2 \pi) \int_{0}^{1 / 2} r^{1-q} d r=\frac{2^{2 q-1} \pi}{2-q}
\end{aligned}
$$

3. Integrando em $A_{1}$. Nesta região temos que $|\zeta| \leq|\zeta-1| ; \log \mathrm{o}, \frac{1}{|\zeta-1|} \leq \frac{1}{|\zeta|}$. Assim,

$$
\begin{aligned}
\iint_{A_{1}} \frac{d \xi d \eta}{|\zeta|^{q}|\zeta-1|^{q}} & \leq \iint_{A_{1}} \frac{1}{|\zeta|^{2 q}} d \xi d \eta \leq \int_{0}^{2 \pi} \int_{1 / 2}^{+\infty} \frac{r}{r^{2 q}} d r d \theta \\
& \leq 2 \pi \int_{1 / 2}^{+\infty} r^{1-2 q} d r=\frac{2^{2 q-2} \pi}{q-1}
\end{aligned}
$$

4. Integrando em $A_{2}$. Nesta área temos que $|\zeta-1| \leq|\zeta| ; \log \frac{1}{|\zeta|} \leq \frac{1}{|\zeta-1|}$. Assim,

$$
\begin{aligned}
\iint_{A_{2}} \frac{d \xi d \eta}{|\zeta|^{q}|\zeta-1|^{q}} & \leq \iint_{A_{2}} \frac{d \xi d \eta}{|\zeta-1|^{2 q}} \leq \int_{0}^{2 \pi} \int_{1 / 2}^{+\infty} \frac{r}{r^{2 q}} d r d \theta \\
& \leq 2 \pi \int_{1 / 2}^{+\infty} r^{1-2 q} d r=\frac{2^{2 q-2} \pi}{q-1}
\end{aligned}
$$


Assim, de 1., 2., 3., 4., temos que

$$
\iint_{\mathbb{R}^{2}} \frac{d \xi d \eta}{|\zeta|^{q}|\zeta-1|^{q}} \leq 2\left[\frac{2^{2 q-1} \pi}{2-q}\right]+2\left[\frac{2^{2 q-2} \pi}{q-1}\right]=\frac{\left(2^{2 q}-2^{2 q-1}\right) \cdot q \pi}{(2-q)(q-1)}<+\infty . \quad(* 4)
$$

Portanto, $\frac{1}{\zeta(\zeta-1)} \in L^{q}(\mathbb{C}), \operatorname{com} 1<q<2$.

Por conseguinte, de $(* 3)$ temos que $\frac{z}{\zeta(\zeta-z)} \in L^{q}(\mathbb{C})$.

Afirmação 2: $|P h(z)| \leq K_{p}\|h\|_{p}|z|^{1-\frac{2}{p}}, \forall z \in \mathbb{C}$.

De fato, para $z=0$ a afirmação é trivial. Vamos, então, considerar $z \neq 0$.

Assim, como $h \in L^{p}(\mathbb{C})$ e $\frac{z}{\zeta(\zeta-z)} \in L^{q}(\mathbb{C})$ com $z \neq 0$, temos pela desigualdade de Hölder em $(* 1)$ que:

$$
|P h(z)| \leq \frac{1}{\pi}\|h\|_{p}\left\|\frac{z}{\zeta(\zeta-z)}\right\|_{q}=\frac{|z|}{\pi}\|h\|_{p}\left\|\frac{1}{\zeta(\zeta-z)}\right\|_{q}
$$

sendo,

$$
\|h\|_{p}=\left(\iint_{\mathbb{R}^{2}}|h(\zeta)|^{p} d \xi d \eta\right)^{1 / p} \text { e }\left\|\frac{1}{\zeta(\zeta-z)}\right\|_{q}=\left(\iint_{\mathbb{R}^{2}} \frac{1}{|\zeta(\zeta-z)|^{q}} d \xi d \eta\right)^{1 / q}
$$

Agora, fazemos a seguinte mudança de variável: tomemos $\zeta=m z, \operatorname{com} m=m_{1}+i m_{2} \in \mathbb{C}$ e $z=x+i y \in \mathbb{C}$; logo, temos que $\xi=m_{1} x-m_{2} y$ e $\eta=m_{1} y+m_{2} x$.

Daí,

$$
\begin{aligned}
d \xi d \eta & =\left|\begin{array}{cc}
\frac{\partial \xi}{\partial m_{1}} & \frac{\partial \eta}{\partial m_{1}} \\
\frac{\partial \xi}{\partial m_{2}} & \frac{\partial \eta}{\partial m_{2}}
\end{array}\right| d m_{1} d m_{2} \\
& =\left|\begin{array}{cc}
x & y \\
-y & x
\end{array}\right| d m_{1} d m_{2} \\
& =|z|^{2} d m_{1} d m_{2} .
\end{aligned}
$$


Assim

$$
\begin{aligned}
\iint_{\mathbb{R}^{2}} \frac{d \xi d \eta}{|\zeta(\zeta-z)|^{q}} & =\iint_{\mathbb{R}^{2}} \frac{|z|^{2} d m_{1} d m_{2}}{|m z(m z-z)|^{q}}=\iint_{\mathbb{R}^{2}} \frac{|z|^{2} d m_{1} d m_{2}}{|z|^{2 q}|m(m-1)|^{q}} \\
& =|z|^{2-2 q} \iint_{\mathbb{R}^{2}} \frac{d m_{1} d m_{2}}{|m(m-1)|^{q}} \\
& \leq \frac{|z|^{2-2 q} \cdot\left(2^{2 q}-2^{2 q-1}\right) \cdot q \cdot \pi}{(2-q)(q-1)} . \quad(\text { por } \quad(* 4))
\end{aligned}
$$

Logo,

$$
\begin{aligned}
\left\|\frac{1}{\zeta(\zeta-z)}\right\|_{q} & =\left(\iint_{\mathbb{R}^{2}} \frac{d \xi d \eta}{\left.\zeta(\zeta-z)\right|^{q}}\right)^{1 / q} \\
& \leq\left(\frac{|z|^{2-2 q} \cdot\left(2^{2 q}-2^{2 q-1}\right) \cdot q \cdot \pi}{(2-q)(q-1)}\right)^{1 / q} \\
& \leq \frac{|z|^{\frac{2}{q}-2} \cdot\left(2^{2 q}-2^{2 q-1}\right)^{1 / q} \cdot q^{1 / q} \cdot \pi^{1 / q}}{(2-q)^{1 / q}(q-1)^{1 / q}} .
\end{aligned}
$$

Assim, substituindo em $(* 5)$ temos

$$
\begin{aligned}
|P h(z)| & \leq \frac{|z|}{\pi}\|h\|_{p} \frac{|z|^{\frac{2}{q}-2} \cdot\left(2^{2 q}-2^{2 q-1}\right)^{1 / q} \cdot q^{1 / q} \cdot \pi^{1 / q}}{(2-q)^{1 / q}(q-1)^{1 / q}} \\
& \leq|z|^{1-\frac{2}{p}}\|h\|_{p}\left(2^{\frac{2 p}{p-1}}-2^{\frac{p+1}{p-1}}\right)^{1-\frac{1}{p}} \cdot \pi^{1-\frac{1}{p}} \cdot\left(\frac{p(p-1)}{p-2}\right)^{1-\frac{1}{p}} \\
& \leq K_{p}\|h\|_{p} \cdot|z|^{1-\frac{2}{p}}
\end{aligned}
$$

sendo $K_{p}=\left(2^{\frac{2 p}{p-1}}-2^{\frac{p+1}{p-1}}\right)^{1-\frac{1}{p}} \cdot \pi^{1-\frac{1}{p}} \cdot\left(\frac{p(p-1)}{p-2}\right)^{1-\frac{1}{p}}$

Portanto,

$$
|P h(z)| \leq K_{p}\|h\|_{p}|z|^{1-\frac{2}{p}},
$$

para todo $z \in \mathbb{C}$. 
Afirmação 3: $\left|P h\left(z_{2}\right)-P h\left(z_{1}\right)\right| \leq K_{p}\|h\|_{p}\left|z_{2}-z_{1}\right|^{1-\frac{2}{p}}, \forall z_{1}, z_{2} \in \mathbb{C}$.

De fato, primeiro definamos a função $h_{1}(w)=h\left(w+z_{1}\right), \forall w=\mu+i \nu \in \mathbb{C}$, com $z_{1}=x_{1}+i y_{1}$ ponto fixado em $\mathbb{C}$. Daí, como

$$
\iint_{\mathbb{R}^{2}} h_{1}(w) d \mu d \nu=\iint_{\mathbb{R}^{2}} h\left(w+z_{1}\right) d \mu d \nu
$$

temos que, $h_{1} \in L^{p}(\mathbb{C}) \Leftrightarrow h \in L^{p}(\mathbb{C})$.

Logo, fazemos a mudança de variáveis $\zeta=w+z_{1}$; temos que $\xi+i \eta=\left(\mu+x_{1}\right)+i\left(\nu+y_{1}\right)$; daí, $d \xi d \eta=d \mu d \nu$.

Assim,

$$
\begin{aligned}
P h\left(z_{2}\right)-P h\left(z_{1}\right) & =-\frac{1}{\pi} \iint_{\mathbb{R}^{2}} h(\zeta) \cdot\left(\frac{1}{\zeta-z_{2}}-\frac{1}{\zeta}\right) d \xi d \eta+\frac{1}{\pi} \iint_{\mathbb{R}^{2}} h(\zeta) \cdot\left(\frac{1}{\zeta-z_{1}}-\frac{1}{\zeta}\right) d \xi d \eta \\
& =-\frac{1}{\pi} \iint_{\mathbb{R}^{2}} h(\zeta) \cdot\left(\frac{1}{\zeta-z_{2}}-\frac{1}{\zeta-z_{1}}\right) d \xi d \eta \\
& =-\frac{1}{\pi} \iint_{\mathbb{R}^{2}} h\left(w+z_{1}\right) \cdot\left(\frac{1}{w+z_{1}-z_{2}}-\frac{1}{w}\right) d \mu d \nu \\
& =-\frac{1}{\pi} \iint_{\mathbb{R}^{2}} h_{1}(w) \cdot\left(\frac{1}{w-\left(z_{2}-z_{1}\right)}-\frac{1}{w}\right) d \mu d \nu \\
& =P h_{1}\left(z_{2}-z_{1}\right) . \quad(\text { por }(1.15))
\end{aligned}
$$

Portanto,

$$
\begin{aligned}
\left|P h\left(z_{2}\right)-P h\left(z_{1}\right)\right| & =\left|P h_{1}\left(z_{2}-z_{1}\right)\right| \leq K_{p}\left\|h_{1}\right\|_{p}\left|z_{2}-z_{1}\right|^{1-\frac{2}{p}} \quad \text { (por Afirmação 2) } \\
& \leq K_{p}\left\|h_{1}\right\|_{p}\left|z_{2}-z_{1}\right|^{1-\frac{2}{p}}
\end{aligned}
$$

para todo $z_{1}, z_{2} \in \mathbb{C}$. 


\subsection{Mudança de variáveis em campos vetoriais}

Definição 1.26 (Campo vetorial complexo). Seja $\Omega$ uma variedade diferenciável. Um campo vetorial sobre $\Omega$ é uma aplicação $\mathbb{C}$ - linear

$$
\begin{aligned}
L: C^{\infty}(\Omega ; \mathbb{C}) & \rightarrow C^{\infty}(\Omega ; \mathbb{C}) \\
f & \mapsto L f
\end{aligned}
$$

que satisfaz a regra de Leibniz:

$$
L(f \cdot g)=L(f) \cdot g+f \cdot L(g),
$$

para todo $f, g \in C^{\infty}(\Omega ; \mathbb{C})$.

Agora, considere o campo vetorial $L: C^{\infty}(\Omega ; \mathbb{C}) \rightarrow C^{\infty}(\Omega ; \mathbb{C})$ dado por

$$
L=a(x, y) \frac{\partial}{\partial x}+b(x, y) \frac{\partial}{\partial y}
$$

$\operatorname{com} a, b \in C^{\infty}(\Omega ; \mathbb{C})$

Sejam $\Omega$ e $\tilde{\Omega}$ abertos de $\mathbb{R}^{2}$. Daqui em diante, em $\Omega$ denotaremos um elemento por $(x, y)$, e em $\tilde{\Omega}$ por $(u, v)$.

Assim, considere $f: \Omega \rightarrow \tilde{\Omega}$ um difeomorfismo $C^{\infty}$ dado por

$$
f(x, y)=\left(f_{1}(x, y), f_{2}(x, y)\right)=(u, v)
$$

$\forall(x, y) \in \Omega$.

Seja $\phi \in C^{\infty}(\tilde{\Omega} ; \mathbb{C})$, daí, $\phi \circ f \in C^{\infty}(\Omega ; \mathbb{C})$. Logo, aplicando o campo vetorial $L$ em $\phi \circ f$ temos

$$
L(\phi \circ f)=a(x, y) \frac{\partial}{\partial x}(\phi \circ f)+b(x, y) \frac{\partial}{\partial y}(\phi \circ f) .
$$


Assim,

$$
\begin{aligned}
& L(\phi \circ f)(x, y)=a(x, y)\left[\frac{\partial \phi}{\partial u}(f(x, y)) \cdot \frac{\partial f_{1}}{\partial x}(x, y)+\frac{\partial \phi}{\partial v}(f(x, y)) \cdot \frac{\partial f_{2}}{\partial x}(x, y)\right] \\
& +b(x, y)\left[\frac{\partial \phi}{\partial u}(f(x, y)) \cdot \frac{\partial f_{1}}{\partial y}(x, y)+\frac{\partial \phi}{\partial v}(f(x, y)) \cdot \frac{\partial f_{2}}{\partial y}(x, y)\right] \\
& =\left[a(x, y) \frac{\partial f_{1}}{\partial x}(x, y)+b(x, y) \frac{\partial f_{1}}{\partial y}(x, y)\right] \frac{\partial \phi}{\partial u}(f(x, y)) \\
& +\left[a(x, y) \frac{\partial f_{2}}{\partial x}(x, y)+b(x, y) \frac{\partial f_{2}}{\partial y}(x, y)\right] \frac{\partial \phi}{\partial v}(f(x, y)) \\
& =\left(L f_{1}\right)(x, y) \cdot \frac{\partial \phi}{\partial u}(f(x, y))+\left(L f_{2}\right)(x, y) \cdot \frac{\partial \phi}{\partial v}(f(x, y)) \\
& =\left(L f_{1}\right) \circ\left(f^{-1} \circ f\right)(x, y) \cdot \frac{\partial \phi}{\partial u}(f(x, y))+\left(L f_{2}\right) \circ\left(f^{-1} \circ f\right)(x, y) \cdot \frac{\partial \phi}{\partial v}(f(x, y)) \\
& =\left(\left(L f_{1}\right) \circ f^{-1}\right)(f(x, y)) \cdot \frac{\partial \phi}{\partial u}(f(x, y))+\left(\left(L f_{2}\right) \circ f^{-1}\right)(f(x, y)) \cdot \frac{\partial \phi}{\partial v}(f(x, y)) \\
& =\left(\left(L f_{1}\right) \circ f^{-1}\right)(u, v) \cdot \frac{\partial \phi}{\partial u}(f(x, y))+\left(\left(L f_{2}\right) \circ f^{-1}\right)(u, v) \cdot \frac{\partial \phi}{\partial v}(f(x, y)) \\
& =\left(\left(\left(L f_{1}\right) \circ f^{-1}\right)(u, v) \cdot \frac{\partial \phi}{\partial u}+\left(\left(L f_{2}\right) \circ f^{-1}\right)(u, v) \cdot \frac{\partial \phi}{\partial v}\right) f(x, y),
\end{aligned}
$$

para todo $(x, y) \in \Omega$.

Portanto,

$$
L(\phi \circ f)=\left(L f_{1}\right) \circ f^{-1}(u, v) \cdot\left[\frac{\partial \phi}{\partial u} \circ f\right]+\left(L f_{2}\right) \circ f^{-1}(u, v) \cdot\left[\frac{\partial \phi}{\partial v} \circ f\right]
$$

Agora, definamos o campo vetorial $\tilde{L}: C^{\infty}(\tilde{\Omega} ; \mathbb{C}) \rightarrow C^{\infty}(\tilde{\Omega} ; \mathbb{C})$ dado por

$$
\tilde{L}=\left(L f_{1}\right) \circ f^{-1}(u, v) \cdot \frac{\partial}{\partial u}+\left(L f_{2}\right) \circ f^{-1}(u, v) \cdot \frac{\partial}{\partial v} .
$$

Assim, seja $\phi \in C^{\infty}(\tilde{\Omega} ; \mathbb{C})$, em (1.19) temos

$$
\tilde{L} \phi=\left(L f_{1}\right) \circ f^{-1}(u, v) \cdot \frac{\partial \phi}{\partial u}+\left(L f_{2}\right) \circ f^{-1}(u, v) \cdot \frac{\partial \phi}{\partial v} .
$$


Dos resultados anteriores, temos a seguinte proposição:

Proposição 1.3. Sejam $\phi \in C^{\infty}(\tilde{\Omega} ; \mathbb{C}), \psi \in C^{\infty}(\Omega ; \mathbb{C})$ e $f: \Omega \rightarrow \tilde{\Omega}$ um difeomorfismo. Então:

1. $L(\phi \circ f)=(\tilde{L} \phi) \circ f$

2. $(L \psi) \circ f^{-1}=\tilde{L}\left(\psi \circ f^{-1}\right)$.

Demonstração.

Para (1):

Segue das equações (1.18) e (1.20).

Para (2):

Considere $\psi=\phi \circ f \in C^{\infty}(\Omega ; \mathbb{C}), \log \mathrm{t}$ temos $\phi=\psi \circ f^{-1} \in C^{\infty}(\tilde{\Omega} ; \mathbb{C})$.

Assim, em (1) temos

$$
L \psi=\tilde{L}\left(\psi \circ f^{-1}\right) \circ f
$$

portanto,

$$
(L \psi) \circ f^{-1}=\tilde{L}\left(\psi \circ f^{-1}\right) .
$$

Definição 1.27 (Integral primeira). Dizemos que o campo vetorial $L$ tem integral primeira num aberto $\Omega \subset \mathbb{R}^{2}$, se existe uma função diferenciável $z: \Omega \rightarrow \mathbb{C}$ tal que $L z=0$ em $\Omega$ e dz $(p) \neq 0, \forall p \in \Omega$. 
Capítulo

2

\section{Princípio da similaridade para o campo vetorial $\frac{\partial}{\partial \bar{z}}$}

\subsection{Função [a,b]-pseudo-analítica}

Vamos considerar nesta seção equações da forma

$$
\frac{\partial \omega}{\partial \bar{z}}(z)=a(z) \cdot \omega(z)+b(z) \cdot \bar{\omega}(z)
$$

sendo $a(z)$ e $b(z)$ funções de classe $C^{\alpha}\left(\mathbb{R}^{2}\right)$, com $0<\alpha<1$.

Daqui em diante, considere $\Omega$ um domínio (aberto e conexo) limitado em $\mathbb{C}$, com $0 \in \Omega$, a fronteira de $\Omega$ seja uma curva de Jordan regular $C^{1}$ por partes orientada positivamente e as funções $a(z), b(z) \in C_{c}^{\alpha}\left(\mathbb{R}^{2}\right)$, com $0<\alpha<1$. Considere, também, $R>0$ suficientemente grande tal que $\operatorname{Supp}(a), \operatorname{Supp}(b) \subset \bar{D}_{R}$, sendo $\bar{D}_{R}=\{z:|z| \leq R\}$ e $\bar{\Omega} \subset D_{R}$.

Definição 2.1 (Função pseudo-analítica). Dizemos que $\omega(z)$ é uma função $[a, b]$ - pseudoanalítica num dominio $\Omega \subset \mathbb{C}$, se $\omega \in C^{1}(\Omega)$ e $\omega$ satisfaz a equação (2.1) em qualquer ponto de $\Omega$.

Observação 2.1. A combinação linear real de duas funções $[a, b]$-pseudo-analíticas é uma função $[a, b]$-pseudo-analítica. 


\subsubsection{Equação integral}

O tratamento da equação (2.1) é baseado em certas propriedades da integral dupla com valores complexos dada na proposição seguinte:

Proposição 2.1. Seja $f: \mathbb{C} \rightarrow \mathbb{C}$ uma função que se anula em $\mathbb{C} \backslash \bar{D}_{R}$. Suponha que existe uma constante $M \geq 0$ tal que $|f(z)| \leq M, \forall z \in \mathbb{C}$. Então, a função $q: \mathbb{C} \rightarrow \mathbb{C}$ dada por

$$
q(z)=-\frac{1}{\pi} \iint_{\Omega} \frac{f(\zeta)}{\zeta-z} d \xi d \eta
$$

$\operatorname{com} \zeta=\xi+i \eta$, satisfaz

$$
|q(z)| \leq K M, \forall z \in \mathbb{C}
$$

$e$

$$
\left|q\left(z_{1}\right)-q\left(z_{2}\right)\right| \leq K M\left|z_{1}-z_{2}\right|^{\epsilon}, \forall z_{1}, z_{2} \in \mathbb{C}
$$

para cada $0<\epsilon<1$, onde a constante $K$ depende unicamente de $\epsilon e R$.

Demonstração.

Vamos começar mostrando que $|q(z)| \leq K M$.

Para isso, fixe $z \in \mathbb{C}$. Como $\bar{\Omega} \subset D_{R}$, temos que

$$
|q(z)| \leq \frac{1}{\pi} \iint_{\Omega} \frac{|f(\zeta)|}{|\zeta-z|} d \xi d \eta \leq \frac{1}{\pi} \iint_{D_{R}} \frac{|f(\zeta)|}{|\zeta-z|} d \xi d \eta \leq \frac{M}{\pi} \iint_{D_{R}} \frac{d \xi d \eta}{|\zeta-z|}
$$

Observemos a figura 2.1.

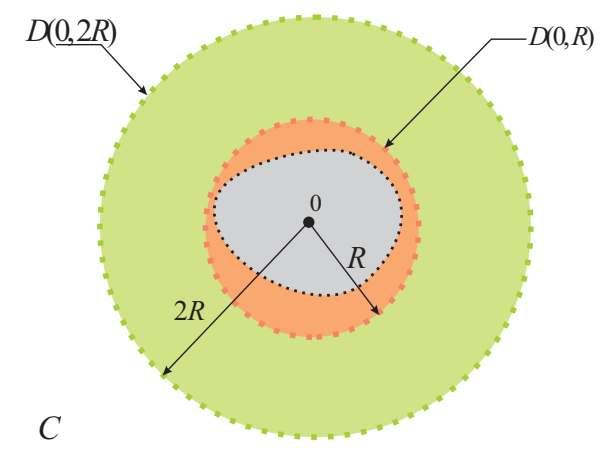

Figura 2.1

Consideremos os seguintes casos: 
- Se $z \in D(0,2 R)$, temos

$$
\begin{aligned}
|q(z)| & \leq \frac{M}{\pi} \iint_{D_{R}} \frac{d \xi d \eta}{|\zeta-z|} \leq \frac{M}{\pi} \iint_{D(z, 4 R)} \frac{d \xi d \eta}{|\zeta-z|} \\
& \leq \frac{M}{\pi} \int_{0}^{2 \pi} \int_{0}^{4 R} \frac{r_{1}}{r_{1}} d r_{1} d \theta=\frac{M}{\pi} \cdot 2 \pi \cdot 4 R \\
& \leq 8 M R .
\end{aligned}
$$

- Se $z \in \mathbb{C} \backslash D(0,2 R)$, temos

$$
\begin{aligned}
|q(z)| & \leq \frac{M}{\pi} \iint_{D_{R}} \frac{d \xi d \eta}{|\zeta-z|} \leq \frac{M}{\pi} \iint_{D_{R}} \frac{d \xi d \eta}{R} \\
& \leq \frac{M}{\pi R} \iint_{D_{R}} d \xi d \eta=\frac{M}{\pi R} \cdot \pi R^{2} \\
& \leq M R \leq 8 M R .
\end{aligned}
$$

Portanto,

$$
|q(z)| \leq K_{1} M,
$$

$\forall z \in \mathbb{C}$, sendo $K_{1}=8 R$.

Finalmente, vamos mostrar que $q(z)$ é Hölder contínua.

Sejam $z_{1}$ e $z_{2}$ pontos fixados em $\mathbb{C}$ tal que $z_{1} \neq z_{2}$. Logo,

$$
\begin{aligned}
q\left(z_{2}\right)-q\left(z_{1}\right)= & -\frac{1}{\pi} \iint_{\Omega} \frac{f(\zeta)}{\zeta-z_{2}} d \xi d \eta+\frac{1}{\pi} \iint_{\Omega} \frac{f(\zeta)}{\zeta-z_{1}} d \xi d \eta \\
= & -\frac{1}{\pi} \iint_{\Omega} f(\zeta)\left(\frac{1}{\zeta-z_{2}}-\frac{1}{\zeta}\right) d \xi d \eta+ \\
& -\left(-\frac{1}{\pi} \iint_{\Omega} f(\zeta)\left(\frac{1}{\zeta-z_{1}}-\frac{1}{\zeta}\right) d \xi d \eta\right) .
\end{aligned}
$$

Agora, definamos a função

$$
\begin{aligned}
\tilde{f}: \mathbb{C} & \longrightarrow \mathbb{C} \\
z & \longmapsto \tilde{f}(z)=\left\{\begin{array}{cl}
f(z) & , \quad z \in \Omega \\
0 & , \quad z \in \mathbb{C} \backslash \Omega
\end{array} .\right.
\end{aligned}
$$


Note que, como $f$ é limitada em $\Omega$ então $\tilde{f}$ é limitada em $\mathbb{C}$. Além disso, como $\tilde{f}$ se anula em $\mathbb{C} \backslash \Omega$ temos que $\tilde{f} \in L^{p}(\mathbb{C}), \forall p \geq 1$. Em particular, $\tilde{f} \in L^{p}(\mathbb{C})$, com $p>2$. Daí, pelo lema 1.5 , temos que

$$
P \tilde{f}(z)=-\frac{1}{\pi} \iint_{\mathbb{R}^{2}} \tilde{f}(\zeta)\left(\frac{1}{\zeta-z}-\frac{1}{\zeta}\right) d \xi d \eta
$$

é Hölder contínua com expoente $1-\frac{2}{p}$, para todo $z \in \mathbb{C}$.

Logo, temos

$$
P \tilde{f}(z)=-\frac{1}{\pi} \iint_{\mathbb{R}^{2}} \tilde{f}(\zeta)\left(\frac{1}{\zeta-z}-\frac{1}{\zeta}\right) d \xi d \eta=-\frac{1}{\pi} \iint_{\Omega} f(\zeta)\left(\frac{1}{\zeta-z}-\frac{1}{\zeta}\right) d \xi d \eta
$$

Assim, de $(* 1)$ e usando o lema 1.5, temos

$$
\begin{aligned}
\left|q\left(z_{2}\right)-q\left(z_{1}\right)\right| & =\left|P \tilde{f}\left(z_{2}\right)-P \tilde{f}\left(z_{1}\right)\right| \leq K_{p}|| f||_{p}\left|z_{2}-z_{1}\right|^{1-\frac{2}{p}} \\
& \leq K_{p}\left(\pi M^{p} R^{2}\right)^{1 / p}\left|z_{2}-z_{1}\right|^{1-\frac{2}{p}} \\
& \leq K_{p} \cdot \pi^{1 / p} M R^{2 / p}\left|z_{2}-z_{1}\right|^{1-\frac{2}{p}}
\end{aligned}
$$

sendo $K_{p}>0$ uma constante que depende de $p$.

Tomemos $\epsilon(p)=1-\frac{2}{p}$; assim, em $(* 2)$ podemos denotar $K_{2}=K_{p} \cdot \pi^{1 / p} R^{2 / p}=K_{p} \cdot \pi^{(1-\epsilon(p)) / 2} R^{1-\epsilon(p)}>0$; daí, $K_{2}$ depende unicamente de $\epsilon$ e $R$. Logo, em $(* 2)$ temos

$$
\left|q\left(z_{2}\right)-q\left(z_{1}\right)\right| \leq K_{2} M\left|z_{2}-z_{1}\right|^{\epsilon} .
$$

Finalmente, tomemos $K=\max \left\{K_{1}, K_{2}\right\}$. Logo,

$$
\left|q\left(z_{2}\right)-q\left(z_{1}\right)\right| \leq K M\left|z_{2}-z_{1}\right|^{\epsilon},
$$

para todo $z_{1}, z_{2} \in \mathbb{C}$ e, além disso, $|q(z)| \leq K M, \forall z \in \mathbb{C}$.

Teorema 2.1. Seja $f: \mathbb{C} \rightarrow \mathbb{C}$ uma função que se anula em $\mathbb{C} \backslash \bar{D}_{R}$. Suponha que existe uma constante $M \geq 0$ tal que $|f(z)| \leq M, \forall z \in \mathbb{C}$. Se $f$ é Hölder contínua em $\Omega$, então a função $q: \mathbb{C} \rightarrow \mathbb{C}$ dada por

$$
q(z)=-\frac{1}{\pi} \iint_{\Omega} \frac{f(\zeta)}{\zeta-z} d \xi d \eta
$$

tem derivadas parciais Hölder contínuas em $\Omega$. 
Além disso,

$$
\frac{\partial q}{\partial \bar{z}}(z)=f(z) \quad e \quad \frac{\partial q}{\partial z}(z)=-\frac{1}{\pi} \iint_{\Omega} \frac{f(\zeta)}{(\zeta-z)^{2}} d \xi d \eta
$$

Demonstração.

Considere a função

$$
h(z)=-\frac{1}{\pi} \iint_{\Omega} \frac{f(\zeta)}{(\zeta-z)^{2}} d \xi d \eta .
$$

Seja $z$ um ponto fixado em $\Omega$ e sejam $\epsilon>0, D_{\epsilon}(z)=\{z:|z|<\epsilon\}$ e $\Omega_{\epsilon}=\Omega \backslash D_{\epsilon}(z)$, $\operatorname{logo}$

$$
\begin{aligned}
h(z) & =-\frac{1}{\pi} \iint_{\Omega} \frac{f(\zeta)}{(\zeta-z)^{2}} d \xi d \eta=-\frac{1}{\pi} \lim _{\epsilon \rightarrow 0} \iint_{\Omega_{\epsilon}} \frac{f(\zeta)}{(\zeta-z)^{2}} d \xi d \eta \\
& =-\frac{1}{\pi} \lim _{\epsilon \rightarrow 0} \iint_{\Omega_{\epsilon}} \frac{f(\zeta)-f(z)}{(\zeta-z)^{2}} d \xi d \eta-f(z) \lim _{\epsilon \rightarrow 0} \frac{1}{\pi} \iint_{\Omega_{\epsilon}} \frac{d \xi d \eta}{(\zeta-z)^{2}} .
\end{aligned}
$$

Por outro lado, temos

$$
\begin{aligned}
\frac{1}{\pi} \iint_{\Omega_{\epsilon}} \frac{d \xi d \eta}{(\zeta-z)^{2}} & =-\frac{1}{\pi} \iint_{\Omega_{\epsilon}} \frac{\partial}{\partial \zeta}\left(\frac{1}{\zeta-z}\right) d \xi d \eta \\
& =\frac{1}{2 \pi i} \int_{\partial \Omega \cup\left\{-\gamma_{\epsilon}\right\}} \frac{1}{\zeta-z} d \bar{\zeta} \quad \text { (pela fórmula (1.8)) } \\
& =\frac{1}{2 \pi i} \int_{\partial \Omega} \frac{1}{\zeta-z} d \bar{\zeta}-\frac{1}{2 \pi i} \int_{\gamma_{\epsilon}} \frac{1}{\zeta-z} d \bar{\zeta} \\
& =\frac{1}{2 \pi i} \int_{\partial \Omega} \frac{1}{\zeta-z} d \bar{\zeta}-\frac{1}{2 \pi i} \int_{0}^{2 \pi} \frac{-i \epsilon e^{-i t}}{\epsilon e^{i t}} d t \\
& =\frac{1}{2 \pi i} \int_{\partial \Omega} \frac{1}{\bar{\zeta}-z} d \bar{\zeta}+\frac{1}{2 \pi} \underbrace{\int_{0}^{2 \pi} e^{-2 i t} d t}_{=0} \\
& =\frac{1}{2 \pi i} \underbrace{\left.\int_{\partial \Omega} d \frac{\bar{\zeta}}{\zeta-z}\right)}_{=0}+\frac{1}{2 \pi i} \int_{\partial \Omega}^{\frac{\bar{\zeta}}{(\zeta-z)^{2}}} d \zeta \\
& =\frac{1}{2 \pi i} \int_{\partial \Omega}^{\frac{\bar{\zeta}}{(\zeta-z)^{2}} d \zeta}
\end{aligned}
$$


Considere:

$$
\phi(z)=\frac{1}{2 \pi i} \int_{\partial \Omega} \frac{\bar{\zeta}}{\zeta-z} d \zeta,
$$

a qual é de classe $C^{1+\alpha}(\bar{\Omega})$ (ver [2], página 87 ).

Daí,

$$
\phi^{\prime}(z)=\frac{1}{2 \pi i} \int_{\partial \Omega} \frac{\bar{\zeta}}{(\zeta-z)^{2}} d \zeta
$$

Assim, de $(* 2)$ temos

$$
\phi^{\prime}(z)=\frac{1}{\pi} \iint_{\Omega_{\epsilon}} \frac{d \xi d \eta}{(\zeta-z)^{2}}
$$

Mostremos que $h \in C^{\alpha}(\Omega)$; para isso, sejam $z_{1}, z_{2} \in \Omega$ com $z_{1} \neq z_{2}$, temos:

$$
\begin{aligned}
& h\left(z_{1}\right)-h\left(z_{2}\right)=-\frac{1}{\pi} \iint_{\Omega} \frac{f(\zeta)}{\left(\zeta-z_{1}\right)^{2}} d \xi d \eta+\frac{1}{\pi} \iint_{\Omega} \frac{f(\zeta)}{\left(\zeta-z_{2}\right)^{2}} d \xi d \eta \\
& =-\frac{1}{\pi} \iint_{\Omega}\left\{\frac{f(\zeta)}{\left(\zeta-z_{1}\right)^{2}}-\frac{f(\zeta)}{\left(\zeta-z_{1}\right)\left(\zeta-z_{2}\right)}+\frac{f(\zeta)}{\left(\zeta-z_{1}\right)\left(\zeta-z_{2}\right)}-\frac{f(\zeta)}{\left(\zeta-z_{2}\right)^{2}}\right\} d \xi d \eta \\
& =\frac{z_{2}-z_{1}}{\pi} \iint_{\Omega} \frac{f(\zeta)}{\left(\zeta-z_{1}\right)^{2}\left(\zeta-z_{2}\right)} d \xi d \eta+\frac{z_{2}-z_{1}}{\pi} \iint_{\Omega} \frac{f(\zeta)}{\left(\zeta-z_{1}\right)\left(\zeta-z_{2}\right)^{2}} d \xi d \eta \\
& =-\frac{z_{1}-z_{2}}{\pi} \iint_{\Omega} \frac{f(\zeta)-f\left(z_{1}\right)}{\left(\zeta-z_{1}\right)^{2}\left(\zeta-z_{2}\right)} d \xi d \eta-\frac{z_{1}-z_{2}}{\pi} \iint_{\Omega} \frac{f(\zeta)-f\left(z_{2}\right)}{\left(\zeta-z_{1}\right)\left(\zeta-z_{2}\right)^{2}} d \xi d \eta+ \\
& -\frac{z_{1}-z_{2}}{\pi} \iint_{\Omega} \frac{f\left(z_{1}\right)}{\left(\zeta-z_{1}\right)^{2}\left(\zeta-z_{2}\right)} d \xi d \eta-\frac{z_{1}-z_{2}}{\pi} \iint_{\Omega} \frac{f\left(z_{2}\right)}{\left(\zeta-z_{1}\right)\left(\zeta-z_{2}\right)^{2}} d \xi d \eta \\
& =-\frac{z_{1}-z_{2}}{\pi} \iint_{\Omega} \frac{f(\zeta)-f\left(z_{1}\right)}{\left(\zeta-z_{1}\right)^{2}\left(\zeta-z_{2}\right)} d \xi d \eta-\frac{z_{1}-z_{2}}{\pi} \iint_{\Omega} \frac{f(\zeta)-f\left(z_{2}\right)}{\left(\zeta-z_{1}\right)\left(\zeta-z_{2}\right)^{2}} d \xi d \eta+ \\
& +f\left(z_{1}\right) \cdot \underbrace{\frac{1}{\pi} \iint_{\Omega} \frac{z_{2}-z_{1}}{\left(\zeta-z_{1}\right)^{2}\left(\zeta-z_{2}\right)} d \xi d \eta}_{(I)}+ \\
& +f\left(z_{2}\right) \cdot \underbrace{\frac{1}{\pi} \iint_{\Omega} \frac{z_{2}-z_{1}}{\left(\zeta-z_{1}\right)\left(\zeta-z_{2}\right)^{2}} d \xi d \eta}_{(I I)} .
\end{aligned}
$$


Em $(I)$ temos

$$
\begin{aligned}
\frac{1}{\pi} \iint_{\Omega} \frac{z_{2}-z_{1}}{\left(\zeta-z_{1}\right)^{2}\left(\zeta-z_{2}\right)} d \xi d \eta= & \frac{1}{\pi\left(z_{1}-z_{2}\right)} \iint_{\Omega} \frac{1}{\zeta-z_{1}} d \xi d \eta-\frac{1}{\pi\left(z_{1}-z_{2}\right)} \iint_{\Omega} \frac{1}{\zeta-z_{2}} d \xi d \eta+ \\
& -\frac{1}{\pi} \iint_{\Omega} \frac{1}{\left(\zeta-z_{1}\right)^{2}} d \xi d \eta .
\end{aligned}
$$

Em $(I I)$ temos

$$
\begin{aligned}
\frac{1}{\pi} \iint_{\Omega} \frac{z_{2}-z_{1}}{\left(\zeta-z_{1}\right)\left(\zeta-z_{2}\right)^{2}} d \xi d \eta= & \frac{1}{\pi\left(z_{1}-z_{2}\right)} \iint_{\Omega} \frac{1}{\zeta-z_{2}} d \xi d \eta-\frac{1}{\pi\left(z_{1}-z_{2}\right)} \iint_{\Omega} \frac{1}{\zeta-z_{1}} d \xi d \eta+ \\
& +\frac{1}{\pi} \iint_{\Omega} \frac{1}{\left(\zeta-z_{2}\right)^{2}} d \xi d \eta .
\end{aligned}
$$

Por outro lado, como a função $g(z)=\bar{z} \in C^{1}(\bar{\Omega} ; \mathbb{C})$, temos por teorema 1.10 e $(* 3)$ que

$$
\begin{aligned}
\bar{z} & =\frac{1}{2 \pi i} \int_{\partial \Omega} \frac{\bar{\zeta}}{\zeta-z} d \zeta-\frac{1}{\pi} \iint_{\Omega} \frac{1}{\zeta-z} d \xi d \eta \\
& =\phi(z)-\frac{1}{\pi} \iint_{\Omega} \frac{1}{\zeta-z} d \xi d \eta .
\end{aligned}
$$

Daí,

$$
\frac{1}{\pi} \iint_{\Omega} \frac{1}{\zeta-z} d \xi d \eta=\phi(z)-\bar{z}
$$

Substituímos $(* 8),(* 4)$ em $(* 6)$, assim temos

$$
\begin{aligned}
\frac{1}{\pi} \iint_{\Omega} \frac{z_{2}-z_{1}}{\left(\zeta-z_{1}\right)^{2}\left(\zeta-z_{2}\right)} d \xi d \eta & =\frac{1}{z_{1}-z_{2}}\left[\phi\left(z_{1}\right)-\bar{z}_{1}\right]-\frac{1}{z_{1}-z_{2}}\left[\phi\left(z_{2}\right)-\bar{z}_{2}\right]-\phi^{\prime}\left(z_{1}\right) \\
& =\frac{\phi\left(z_{1}\right)-\phi\left(z_{2}\right)}{z_{1}-z_{2}}-\frac{\overline{z_{1}-z_{2}}}{z_{1}-z_{2}}-\phi^{\prime}\left(z_{1}\right) .
\end{aligned}
$$

Igualmente, substituímos $(* 8),(* 4)$ em $(* 7)$, assim temos

$$
\begin{aligned}
\frac{1}{\pi} \iint_{\Omega} \frac{z_{2}-z_{1}}{\left(\zeta-z_{1}\right)\left(\zeta-z_{2}\right)^{2}} d \xi d \eta & =\frac{1}{z_{1}-z_{2}}\left[\phi\left(z_{2}\right)-\bar{z}_{2}\right]-\frac{1}{z_{1}-z_{2}}\left[\phi\left(z_{1}\right)-\bar{z}_{1}\right]-\phi^{\prime}\left(z_{2}\right) \\
& =-\frac{\phi\left(z_{1}\right)-\phi\left(z_{2}\right)}{z_{1}-z_{2}}+\frac{\overline{z_{1}-z_{2}}}{z_{1}-z_{2}}+\phi^{\prime}\left(z_{2}\right) .
\end{aligned}
$$


Substituímos $(* 9)$ e $(* 10)$ em $(* 5)$, logo

$$
\begin{aligned}
h\left(z_{1}\right)-h\left(z_{2}\right)= & -\frac{z_{1}-z_{2}}{\pi} \iint_{\Omega} \frac{f(\zeta)-f\left(z_{1}\right)}{\left(\zeta-z_{1}\right)^{2}\left(\zeta-z_{2}\right)} d \xi d \eta-\frac{z_{1}-z_{2}}{\pi} \iint_{\Omega} \frac{f(\zeta)-f\left(z_{2}\right)}{\left(\zeta-z_{1}\right)\left(\zeta-z_{2}\right)^{2}} d \xi d \eta+ \\
& +f\left(z_{1}\right) \cdot \frac{\phi\left(z_{1}\right)-\phi\left(z_{2}\right)}{z_{1}-z_{2}}-f\left(z_{1}\right) \cdot \frac{\overline{z_{1}-z_{2}}}{z_{1}-z_{2}}-f\left(z_{1}\right) \cdot \phi^{\prime}\left(z_{1}\right)+ \\
& -f\left(z_{2}\right) \cdot \frac{\phi\left(z_{1}\right)-\phi\left(z_{2}\right)}{z_{1}-z_{2}}+f\left(z_{2}\right) \cdot \frac{\overline{z_{1}-z_{2}}}{z_{1}-z_{2}}+f\left(z_{2}\right) \cdot \phi^{\prime}\left(z_{2}\right) \\
= & -\frac{z_{1}-z_{2}}{\pi} \iint_{\Omega} \frac{f(\zeta)-f\left(z_{1}\right)}{\left(\zeta-z_{1}\right)^{2}\left(\zeta-z_{2}\right)} d \xi d \eta-\frac{z_{1}-z_{2}}{\pi} \iint_{\Omega} \frac{f(\zeta)-f\left(z_{2}\right)}{\left(\zeta-z_{1}\right)\left(\zeta-z_{2}\right)^{2}} d \xi d \eta+ \\
& +\left[f\left(z_{1}\right)-f\left(z_{2}\right)\right] \cdot\left\{\frac{\phi\left(z_{1}\right)-\phi\left(z_{2}\right)}{z_{1}-z_{2}}-\frac{\left.\frac{z_{1}-z_{2}}{z_{1}-z_{2}}-\phi^{\prime}\left(z_{2}\right)\right\}+}{}\right. \\
& +f\left(z_{1}\right) \cdot\left[\phi^{\prime}\left(z_{2}\right)-\phi^{\prime}\left(z_{1}\right)\right] .
\end{aligned}
$$

Daí,

$$
\begin{aligned}
\left|h\left(z_{1}\right)-h\left(z_{2}\right)\right| \leq & \frac{\left|z_{1}-z_{2}\right|}{\pi} \iint_{\Omega} \frac{\left|f(\zeta)-f\left(z_{1}\right)\right|}{\left|\zeta-z_{1}\right|{ }^{2}\left|\zeta-z_{2}\right|} d \xi d \eta+\frac{\left|z_{1}-z_{2}\right|}{\pi} \iint_{\Omega} \frac{\left|f(\zeta)-f\left(z_{2}\right)\right|}{\left|\zeta-z_{1}\right|\left|\zeta-z_{2}\right|^{2}} d \xi d \eta+ \\
& +\left|f\left(z_{1}\right)-f\left(z_{2}\right)\right| \cdot\left\{\frac{\left|\phi\left(z_{1}\right)-\phi\left(z_{2}\right)\right|}{\left|z_{1}-z_{2}\right|}+\frac{\left|\overline{z_{1}-z_{2}}\right|}{\left|z_{1}-z_{2}\right|}+\left|\phi^{\prime}\left(z_{2}\right)\right|\right\}+ \\
& +\left|f\left(z_{1}\right)\right| \cdot\left|\phi^{\prime}\left(z_{2}\right)-\phi^{\prime}\left(z_{1}\right)\right| .
\end{aligned}
$$

Sabemos que $\phi \in C^{1+\alpha}(\bar{\Omega}) ; \operatorname{logo} \phi^{\prime}(z) \in C^{\alpha}(\bar{\Omega})$. Também, como $\Omega$ é limitado e $\phi^{\prime}(z)$ é contínua em $\bar{\Omega}$, temos que $\phi$ é Lipschitziana com constante de Lipschitz $K_{1}$.

Por outro lado, $f \in C^{\alpha}(\Omega)$, isto é, existe $C>0$ tal que $\left|f\left(z_{1}\right)-f\left(z_{2}\right)\right| \leq C\left|z_{1}-z_{2}\right|^{\alpha}$, $\forall z_{1}, z_{2} \in \Omega$.

Assim, em $(* 11)$ temos

$$
\begin{aligned}
\left|h\left(z_{1}\right)-h\left(z_{2}\right)\right| \leq & C\left|z_{1}-z_{2}\right| \underbrace{\frac{1}{\pi} \iint_{\Omega} \frac{d \xi d \eta}{\left|\zeta-z_{1}\right|^{2-\alpha}\left|\zeta-z_{2}\right|}}_{(I I I)}+C\left|z_{1}-z_{2}\right| \frac{1}{\pi} \iint_{\Omega} \frac{d \xi d \eta}{\left|\zeta-z_{1}\right|\left|\zeta-z_{2}\right|^{2-\alpha}}+ \\
& +C\left|z_{1}-z_{2}\right|^{\alpha}\left\{K_{1}+1+K_{2}\right\}+M C_{1}\left|z_{1}-z_{2}\right|^{\alpha} .
\end{aligned}
$$


Agora, vamos a estimar as integrais $(I I I)$ e $(I V)$. Para isso, fixamos o ponto $z_{1}$ e vamos considerar o disco $D_{1}=B\left(z_{1}, \delta\right)$, sendo $\delta=2\left|z_{1}-z_{2}\right|$, e o disco $D_{0}=B\left(z_{1}, 2 \delta_{0}\right)$ tal que $\bar{\Omega} \subset D_{0}$.

Note que:

- Se $\zeta \in D_{0} \backslash D_{1}$, temos que

$$
\begin{aligned}
& 2\left|\zeta-z_{2}\right| \geq 2\left(\left|\zeta-z_{1}\right|-\left|z_{1}-z_{2}\right|\right) \geq 2\left|\zeta-z_{1}\right|-2\left|z_{1}-z_{2}\right| . \quad \Rightarrow \\
& 2\left|\zeta-z_{2}\right| \geq 2\left|\zeta-z_{1}\right|-\left|\zeta-z_{1}\right| \geq\left|\zeta-z_{1}\right| .
\end{aligned}
$$

Logo, $\frac{1}{\left|\zeta-z_{2}\right|} \leq \frac{2}{\left|\zeta-z_{1}\right|}$

- Se $\zeta \in D_{1}$, temos que $\left|\zeta-z_{1}\right| \leq 2\left|z_{1}-z_{2}\right|=\delta$.

Daí, fazemos a seguinte mudança de variável:

Tomemos $\psi=\psi_{1}+i \psi_{2}=\frac{\zeta-z_{1}}{z_{1}-z_{2}}, \operatorname{com} \zeta=\xi+i \eta, z_{1}=x_{1}+i y_{1}$ e $z_{2}=x_{2}+i y_{2}$. Assim,

$\psi_{1}=\frac{\left(\xi-x_{1}\right)\left(x_{1}-x_{2}\right)+\left(\eta-y_{1}\right)\left(y_{1}-y_{2}\right)}{\left|z_{1}-z_{2}\right|^{2}}$ e $\psi_{2}=\frac{\left(\eta-y_{1}\right)\left(x_{1}-x_{2}\right)-\left(\xi-x_{1}\right)\left(y_{1}-y_{2}\right)}{\left|z_{1}-z_{2}\right|^{2}}$

Logo,

$$
\begin{aligned}
d \psi_{1} d \psi_{2} & =\left|\begin{array}{cc}
\frac{\partial \psi_{1}}{\partial \xi} & \frac{\partial \psi_{2}}{\partial \xi} \\
\frac{\partial \psi_{1}}{\partial \eta} & \frac{\partial \psi_{2}}{\partial \eta}
\end{array}\right| d \xi d \eta=\left|\begin{array}{cc}
\frac{x_{1}-x_{2}}{\left|z_{1}-z_{2}\right|^{2}} & -\frac{y_{1}-y_{2}}{\left|z_{1}-z_{2}\right|^{2}} \\
\frac{y_{1}-y_{2}}{\left|z_{1}-z_{2}\right|^{2}} & \frac{x_{1}-x_{2}}{\left|z_{1}-z_{2}\right|^{2}}
\end{array}\right| d \xi d \eta \\
& =\frac{\left(x_{1}-x_{2}\right)^{2}+\left(y_{1}-y_{2}\right)^{2}}{\left|z_{1}-z_{2}\right|^{4}} d \xi d \eta=\frac{1}{\left|z_{1}-z_{2}\right|^{2}} d \xi d \eta
\end{aligned}
$$

Daí,

$$
d \xi d \eta=\left|z_{1}-z_{2}\right|^{2} d \psi_{1} d \psi_{2}
$$

Também, $\left|\frac{\zeta-z_{2}}{z_{1}-z_{2}}\right|=\left|\frac{\zeta-z_{1}}{z_{1}-z_{2}}+\frac{z_{1}-z_{2}}{z_{1}-z_{2}}\right|=|\psi+1|$. 
Assim, temos

$$
\begin{aligned}
\iint_{D_{1}} \frac{d \xi d \eta}{\left|\zeta-z_{1}\right|^{2-\alpha}\left|\zeta-z_{2}\right|} & =\iint_{\left|\zeta-z_{1}\right| \leq \delta} \frac{\left|\zeta-z_{1}\right|^{\alpha-2}\left|\zeta-z_{2}\right|^{-1}\left|z_{1}-z_{2}\right|^{\alpha-3}}{\left|z_{1}-z_{2}\right|^{\alpha-2}\left|z_{1}-z_{2}\right|^{-1}} d \xi d \eta \\
& =\iint_{|\psi| \leq 2}|\psi|^{\alpha-2}|\psi+1|^{-1}\left|z_{1}-z_{2}\right|^{\alpha-1} d \psi_{1} d \psi_{2} \\
& =\left|z_{1}-z_{2}\right|^{\alpha-1} \iint_{|\psi| \leq 2} \frac{d \psi_{1} d \psi_{2}}{|\psi|^{2-\alpha}|\psi+1|}
\end{aligned}
$$

e

$$
\begin{aligned}
\iint_{D_{1}} \frac{d \xi d \eta}{\left|\zeta-z_{1}\right|\left|\zeta-z_{2}\right|^{2-\alpha}} & =\iint_{\left|\zeta-z_{1}\right| \leq \delta} \frac{\left|\zeta-z_{1}\right|^{-1}\left|\zeta-z_{2}\right|^{\alpha-2}\left|z_{1}-z_{2}\right|^{\alpha-3}}{\left|z_{1}-z_{2}\right|^{-1}\left|z_{1}-z_{2}\right|^{\alpha-2}} d \xi d \eta \\
& =\iint_{|\psi| \leq 2}|\psi|^{-1}|\psi+1|^{\alpha-2}\left|z_{1}-z_{2}\right|^{\alpha-1} d \psi_{1} d \psi_{2} \\
& =\left|z_{1}-z_{2}\right|^{\alpha-1} \iint_{|\psi| \leq 2} \frac{d \psi_{1} d \psi_{2}}{|\psi||\psi+1|^{2-\alpha}}
\end{aligned}
$$

Para (III) temos

$$
\begin{aligned}
\frac{1}{\pi} \iint_{\Omega} \frac{d \xi d \eta}{\left|\zeta-z_{1}\right|^{2-\alpha}\left|\zeta-z_{2}\right|} \leq & \frac{1}{\pi} \iint_{D_{0} \backslash D_{1}} \frac{d \xi d \eta}{\left|\zeta-z_{1}\right|^{2-\alpha}\left|\zeta-z_{2}\right|}+\frac{1}{\pi} \iint_{D_{1}} \frac{d \xi d \eta}{\left|\zeta-z_{1}\right|^{2-\alpha}\left|\zeta-z_{2}\right|} \\
\leq & \underbrace{\frac{1}{\pi} \iint_{D_{0} \backslash D_{1}} \frac{2 d \xi d \eta}{\left|\zeta-z_{1}\right|^{2-\alpha}\left|\zeta-z_{1}\right|}}_{(V)}+ \\
& +\frac{\left|z_{1}-z_{2}\right|^{\alpha-1}}{\pi} \underbrace{\iint_{|\psi| \leq 2} \frac{d \psi_{1} d \psi_{2}}{|\psi-\alpha| \psi+1 \mid}}_{(V I)}
\end{aligned}
$$

Estimemos a integral em $(V)$

$$
\begin{aligned}
\frac{1}{\pi} \iint_{D_{0} \backslash D_{1}} \frac{2 d \xi d \eta}{\left|\zeta-z_{1}\right|^{2-\alpha}\left|\zeta-z_{1}\right|} & =\frac{2}{\pi} \int_{0}^{2 \pi} \int_{\delta}^{2 \delta_{0}} \frac{r_{1} d r_{1} d \theta}{r_{1}^{3-\alpha}}=\frac{4}{\alpha-1}\left(2^{\alpha-1} \delta_{0}^{\alpha-1}-\delta^{\alpha-1}\right) \\
& =\frac{2^{2}}{1-\alpha} \delta^{\alpha-1}-\frac{2^{1+\alpha}}{1-\alpha} \cdot \frac{1}{\delta_{0}^{1-\alpha}} \leq \frac{2^{2}}{1-\alpha}\left|z_{1}-z_{2}\right|^{\alpha-1}
\end{aligned}
$$


Estimemos a integral em (VI)

$$
\begin{aligned}
\iint_{|\psi| \leq 2} \frac{d \psi_{1} d \psi_{2}}{|\psi|^{2-\alpha}|\psi+1|} & =\iint_{|\psi| \leq \frac{1}{2}} \frac{d \psi_{1} d \psi_{2}}{|\psi|^{2-\alpha}|\psi+1|}+\iint_{\frac{1}{2} \leq|\psi| \leq 2} \frac{d \psi_{1} d \psi_{2}}{|\psi|^{2-\alpha}|\psi+1|} \\
& \leq \iint_{|\psi| \leq \frac{1}{2}} \frac{d \psi_{1} d \psi_{2}}{|\psi|^{2-\alpha}(1-|\psi|)}+\iint_{\frac{1}{2} \leq|\psi| \leq 2} \frac{d \psi_{1} d \psi_{2}}{|\psi|^{3-\alpha}} \\
& \leq \int_{0}^{2 \pi} \int_{0}^{1 / 2} \frac{r_{1} d r_{1} d \theta}{r_{1}^{2-\alpha}\left(1-r_{1}\right)}+\int_{0}^{2 \pi} \int_{1 / 2}^{2} \frac{r_{1} d r_{1} d \theta}{r_{1}^{3-\alpha}} \\
& \leq \int_{0}^{2 \pi} \int_{0}^{1 / 2} \frac{d r_{1} d \theta}{r_{1}^{1-\alpha}\left(1-r_{1}\right)}+\int_{0}^{2 \pi} \int_{1 / 2}^{2} r_{1}^{\alpha-2} d r_{1} d \theta \\
& \leq \frac{2^{2-\alpha} \pi}{\alpha}+\frac{2 \pi}{1-\alpha}\left[2^{1-\alpha}-\frac{1}{2^{1-\alpha}}\right] .
\end{aligned}
$$

Assim, em (*15) temos

$$
\begin{aligned}
\frac{1}{\pi} \iint_{\Omega} \frac{d \xi d \eta}{\left|\zeta-z_{1}\right|^{2-\alpha}\left|\zeta-z_{2}\right|} \leq & \frac{2^{2}}{1-\alpha} \cdot\left|z_{1}-z_{2}\right|^{\alpha-1}+\frac{\left|z_{1}-z_{2}\right|^{\alpha-1}}{\pi}\left\{\frac{2^{2-\alpha} \pi}{\alpha}+\right. \\
& \left.+\frac{2 \pi}{1-\alpha}\left[2^{1-\alpha}-\frac{1}{2^{1-\alpha}}\right]\right\} \\
\leq & \left\{\frac{\alpha\left(2^{2}-2^{\alpha}\right)+2^{2-\alpha}}{(1-\alpha) \alpha}\right\}\left|z_{1}-z_{2}\right|^{\alpha-1}
\end{aligned}
$$

Para $(I V)$ temos

$$
\begin{aligned}
\frac{1}{\pi} \iint_{\Omega} \frac{d \xi d \eta}{\left|\zeta-z_{1}\right|\left|\zeta-z_{2}\right|^{2-\alpha}} \leq & \frac{1}{\pi} \iint_{D_{0} \backslash D_{1}} \frac{d \xi d \eta}{\left|\zeta-z_{1}\right|\left|\zeta-z_{2}\right|^{2-\alpha}}+\frac{1}{\pi} \iint_{D_{1}} \frac{d \xi d \eta}{\left|\zeta-z_{1}\right|\left|\zeta-z_{2}\right|^{2-\alpha}} \\
\leq & \underbrace{\frac{1}{\pi} \iint_{D_{0} \backslash D_{1}} \frac{2^{2-\alpha} d \xi d \eta}{\left|\zeta-z_{1}\right|\left|\zeta-z_{1}\right|^{2-\alpha}}}_{(V I I)}+ \\
& +\frac{\left|z_{1}-z_{2}\right|^{\alpha-1}}{\pi} \underbrace{\iint_{|\psi| \leq 2} \frac{d \psi_{1} d \psi_{2}}{|\psi||\psi+1|^{2-\alpha}}}_{(V I I I)} .
\end{aligned}
$$


Estimemos a integral em (VII)

$$
\begin{aligned}
\frac{1}{\pi} \iint_{D_{0} \backslash D_{1}} \frac{2^{2-\alpha} d \xi d \eta}{\left|\zeta-z_{1}\right|\left|\zeta-z_{1}\right|^{2-\alpha}} & =\frac{2^{2-\alpha}}{\pi} \int_{0}^{2 \pi} \int_{\delta}^{2 \delta_{0}} \frac{r_{1} d r_{1} d \theta}{r_{1}^{3-\alpha}}=\frac{2^{3-\alpha}}{\alpha-1}\left(2^{\alpha-1} \delta_{0}^{\alpha-1}-\delta^{\alpha-1}\right) \\
& =\frac{2^{3-\alpha}}{1-\alpha} \delta^{\alpha-1}-\frac{2^{2}}{1-\alpha} \cdot \frac{1}{\delta_{0}^{1-\alpha}} \\
& \leq \frac{2^{3-\alpha}}{1-\alpha} \cdot\left|z_{1}-z_{2}\right|^{\alpha-1} .
\end{aligned}
$$

Estimemos a integral em (VIII)

$$
\begin{aligned}
\iint_{|\psi| \leq 2} \frac{d \psi_{1} d \psi_{2}}{|\psi||\psi+1|^{2-\alpha}} & =\iint_{|\psi| \leq \frac{1}{2}} \frac{d \psi_{1} d \psi_{2}}{|\psi||\psi+1|^{2-\alpha}}+\iint_{\frac{1}{2} \leq|\psi| \leq 2} \frac{d \psi_{1} d \psi_{2}}{|\psi||\psi+1|^{2-\alpha}} \\
& \leq \iint_{|\psi| \leq \frac{1}{2}} \frac{d \psi_{1} d \psi_{2}}{|\psi|(1-|\psi|)^{2-\alpha}}+\iint_{\frac{1}{2} \leq|\psi| \leq 2} \frac{d \psi_{1} d \psi_{2}}{|\psi|^{3-\alpha}} \\
& \leq \int_{0}^{2 \pi} \int_{0}^{1 / 2} \frac{r_{1} d r_{1} d \theta}{r_{1}\left(1-r_{1}\right)^{2-\alpha}}+\int_{0}^{2 \pi} \int_{1 / 2}^{2} \frac{r_{1} d r_{1} d \theta}{r_{1}^{3-\alpha}} \\
& \leq \int_{0}^{2 \pi} \int_{0}^{1 / 2} 2^{2-\alpha} d r_{1} d \theta+\int_{0}^{2 \pi} \int_{1 / 2}^{2} r_{1}^{\alpha-2} d r_{1} d \theta \\
& \leq 2^{2-\alpha} \pi+\frac{2 \pi}{1-\alpha}\left[2^{1-\alpha}-\frac{1}{2^{1-\alpha}}\right] .
\end{aligned}
$$

Assim, em (*17) temos

$$
\begin{aligned}
\frac{1}{\pi} \iint_{\Omega} \frac{d \xi d \eta}{\left|\zeta-z_{1}\right|\left|\zeta-z_{2}\right|^{2-\alpha}} \leq & \frac{2^{3-\alpha}}{1-\alpha} \cdot\left|z_{1}-z_{2}\right|^{\alpha-1}+\frac{\left|z_{1}-z_{2}\right|^{\alpha-1}}{\pi}\left\{2^{2-\alpha} \pi+\right. \\
& \left.+\frac{2 \pi}{1-\alpha}\left[2^{1-\alpha}-\frac{1}{2^{1-\alpha}}\right]\right\} \\
\leq & \left\{\frac{2^{4-\alpha}-2^{\alpha}-\alpha \cdot 2^{2-\alpha}}{1-\alpha}\right\}\left|z_{1}-z_{2}\right|^{\alpha-1} .
\end{aligned}
$$


Substituindo $(* 16)$ e $(* 18)$ em $(* 12)$ temos

$$
\begin{aligned}
\left|h\left(z_{1}\right)-h\left(z_{2}\right)\right| \leq & C\left|z_{1}-z_{2}\right| \cdot \frac{1}{\alpha(1-\alpha)} \cdot\left\{\alpha\left(2^{2}-2^{\alpha}\right)+2^{2-\alpha}\right\} \cdot\left|z_{1}-z_{2}\right|^{\alpha-1}+ \\
& +C\left|z_{1}-z_{2}\right| \cdot \frac{1}{1-\alpha} \cdot\left(2^{4-\alpha}-2^{\alpha}-\alpha \cdot 2^{2-\alpha}\right) \cdot\left|z_{1}-z_{2}\right|^{\alpha-1}+ \\
& +C\left|z_{1}-z_{2}\right|^{\alpha}\left\{K_{1}+1+K_{2}\right\}+M \cdot C_{1}\left|z_{1}-z_{2}\right|^{\alpha} \\
\leq & {\left[\frac{C}{\alpha(1-\alpha)} \cdot\left\{\alpha\left(2^{2}-2^{\alpha}\right)+2^{2-\alpha}\right\}+C\left(K_{1}+1+K_{2}\right)+M C_{1}+\right.} \\
& \left.+\frac{C}{1-\alpha} \cdot\left(2^{4-\alpha}-2^{\alpha}-\alpha \cdot 2^{2-\alpha}\right)\right] \cdot\left|z_{1}-z_{2}\right|^{\alpha} .
\end{aligned}
$$

Escolha

$$
\begin{aligned}
K= & \frac{C}{\alpha(1-\alpha)} \cdot\left\{\alpha\left(2^{2}-2^{\alpha}\right)+2^{2-\alpha}\right\}+C\left(K_{1}+1+K_{2}\right)+M C_{1}+ \\
& +\frac{C}{1-\alpha} \cdot\left(2^{4-\alpha}-2^{\alpha}-\alpha \cdot 2^{2-\alpha}\right)>0
\end{aligned}
$$

Assim, temos

$$
\left|h\left(z_{1}\right)-h\left(z_{2}\right)\right| \leq K\left|z_{1}-z_{2}\right|^{\alpha}
$$

Portanto, $h \in C^{\alpha}(\Omega)$.

Agora, vamos mostrar a validade de (2.5). Para isso, sejam $w, z \in \Omega$, com $w \neq z$; 
temos:

$$
\begin{aligned}
& \frac{q(w)-q(z)}{w-z}-h(z)=\frac{1}{w-z}\left[-\frac{1}{\pi} \iint_{\Omega} \frac{f(\zeta)}{\zeta-w} d \xi d \eta+\frac{1}{\pi} \iint_{\Omega} \frac{f(\zeta)}{\zeta-z} d \xi d \eta\right]+ \\
& +\frac{1}{\pi} \iint_{\Omega} \frac{f(\zeta)}{(\zeta-z)^{2}} d \xi d \eta \\
& =-\frac{1}{(w-z) \pi} \iint_{\Omega}\left[\frac{1}{\zeta-w}-\frac{1}{\zeta-z}-\frac{w-z}{(\zeta-z)^{2}}\right] f(\zeta) d \xi d \eta \\
& =-\frac{w-z}{\pi} \iint_{\Omega} \frac{f(\zeta)}{(\zeta-w)(\zeta-z)^{2}} d \xi d \eta \\
& =-\frac{w-z}{\pi} \iint_{\Omega} \frac{f(\zeta)-f(z)}{(\zeta-w)(\zeta-z)^{2}} d \xi d \eta-\frac{f(z)}{\pi} \iint_{\Omega} \frac{w-z}{(\zeta-w)(\zeta-z)^{2}} d \xi d \eta \\
& =-\frac{w-z}{\pi} \iint_{\Omega} \frac{f(\zeta)-f(z)}{(\zeta-w)(\zeta-z)^{2}} d \xi d \eta-f(z) . \\
& \cdot\left[\frac{\phi(z)-\phi(w)}{z-w}-\frac{\overline{z-w}}{z-w}-\phi^{\prime}(z)\right] \quad(\operatorname{por}(* 9)) \\
& =-\frac{w-z}{\pi} \iint_{\Omega} \frac{f(\zeta)-f(z)}{(\zeta-w)(\zeta-z)^{2}} d \xi d \eta-f(z) \cdot \frac{\phi(z)-\phi(w)}{z-w}+ \\
& +\frac{\overline{z-w}}{z-w} \cdot f(z)+\phi^{\prime}(z) \cdot f(z) .
\end{aligned}
$$

Daí,

$$
\begin{aligned}
\frac{q(w)-q(z)}{w-z}-h(z)-\frac{\overline{z-w}}{z-w} \cdot f(z)= & -\frac{w-z}{\pi} \iint_{\Omega} \frac{f(\zeta)-f(z)}{(\zeta-w)(\zeta-z)^{2}} d \xi d \eta+ \\
& -f(z) \cdot \frac{\phi(z)-\phi(w)}{z-w}+\phi^{\prime}(z) \cdot f(z) .
\end{aligned}
$$


Logo,

$$
\begin{aligned}
&\left|\frac{q(w)-q(z)}{w-z}-h(z)-\frac{\overline{z-w}}{z-w} \cdot f(z)\right| \leq \frac{|w-z|}{\pi} \iint_{\Omega} \frac{|f(\zeta)-f(z)|}{|\zeta-w||\zeta-z|^{2}} d \xi d \eta+ \\
&+|f(z)| \cdot\left|\phi^{\prime}(z)-\frac{\phi(z)-\phi(w) \mid}{z-w}\right| \\
& \leq \frac{C|w-z|}{\pi} \iint_{\Omega} \frac{d \xi d \eta}{|\zeta-w||\zeta-z|^{2-\alpha}}+ \\
&+M \cdot\left|\phi^{\prime}(z)-\frac{\phi(z)-\phi(w) \mid}{z-w}\right| \\
& \leq\left.C \cdot|w-z| \cdot\left|\frac{2^{4-\alpha}-2^{\alpha}-\alpha \cdot 2^{2-\alpha}}{1-\alpha}\right||w-z|^{\alpha-1}\right]+ \\
&+M \cdot\left|\phi^{\prime}(z)-\frac{\phi(z)-\phi(w) \mid}{z-w}\right| \\
& \leq \frac{C}{1-\alpha}\left(2^{4-\alpha}-2^{\alpha}-\alpha \cdot 2^{2-\alpha}\right)|w-z|^{\alpha}+ \\
&+M\left|\phi^{\prime}(z)-\frac{\phi(z)-\phi(w)}{z-w}\right| \cdot \\
& \text { por }(* 18)
\end{aligned}
$$

Escolha $\tilde{K}=\frac{C}{1-\alpha}\left(2^{4-\alpha}-2^{\alpha}-\alpha \cdot 2^{2-\alpha}\right)$. Assim,

$$
\left|\frac{q(w)-q(z)}{w-z}-h(z)-\frac{\overline{z-w}}{z-w} \cdot f(z)\right| \leq \tilde{K}|w-z|^{\alpha}+M \cdot\left|\phi^{\prime}(z)-\frac{\phi(z)-\phi(w)}{z-w}\right| .
$$

Agora, fazemos $w \rightarrow z$ e como $w-z=|w-z| e^{i \theta}$, com $\theta \in[0,2 \pi]$, temos:

$$
\begin{gathered}
\lim _{w \rightarrow z}\left|\frac{q(w)-q(z)}{w-z}-h(z)-\frac{|\overline{w-z}| e^{-i \theta}}{|w-z| e^{i \theta}} f(z)\right| \leq \tilde{K} \lim _{w \rightarrow z}|w-z|^{\alpha}+M \lim _{w \rightarrow z}\left|\phi^{\prime}(z)-\frac{\phi(w)-\phi(z)}{w-z}\right| \Rightarrow \\
\lim _{w \rightarrow z}\left|\frac{q(w)-q(z)}{w-z}-h(z)-e^{-i 2 \theta} \cdot f(z)\right|=0 \\
\lim _{w \rightarrow z}\left\{\frac{q(w)-q(z)}{w-z}\right\}=h(z)+e^{-i 2 \theta} \cdot f(z) .
\end{gathered} \Rightarrow
$$


Assim,

- Se $w=w_{1}+i w_{2}$ tende a $z=x+i y$ ao longo da reta horizontal $w_{2}=y(\theta=0)$, temos

$$
\frac{\partial q}{\partial x}(z)=h(z)+f(z)
$$

- Se $w=w_{1}+i w_{2}$ tende a $z=x+i y$ ao longo da reta vertical $w_{1}=x(\theta=\pi / 2)$, temos

$$
-i \frac{\partial q}{\partial y}(z)=h(z)-f(z)
$$

Logo,

$$
\frac{\partial q}{\partial y}(z)=i h(z)-i f(z)
$$

Finalmente, temos

$$
\frac{\partial q}{\partial \bar{z}}(z)=\frac{1}{2}\left(\frac{\partial}{\partial x}+i \frac{\partial}{\partial y}\right) q(z)=f(z)
$$

e

$$
\frac{\partial q}{\partial z}(z)=\frac{1}{2}\left(\frac{\partial}{\partial x}-i \frac{\partial}{\partial y}\right) q(z)=h(z)=-\frac{1}{\pi} \iint_{\Omega} \frac{f(\zeta)}{(\zeta-z)^{2}} d \xi d \eta
$$

Proposição 2.2. Seja $\omega: \Omega \rightarrow \mathbb{C}$ uma função contínua e limitada em um domínio $\Omega$. Então, $\omega(z)$ é $[a, b]$-pseudo-analítica em $\Omega$ se, e somente se, a função definida por

$$
f(z)=\omega(z)+\frac{1}{\pi} \iint_{\Omega} \frac{a(\zeta) \cdot \omega(\zeta)+b(\zeta) \cdot \bar{\omega}(\zeta)}{\zeta-z} d \xi d \eta
$$

é analítica em $\Omega$.

Demonstração.

Definamos

$$
\begin{aligned}
g: \Omega & \rightarrow \mathbb{C} \\
z & \mapsto g(z)=a(z) \cdot \omega(z)+b(z) \cdot \bar{\omega}(z)
\end{aligned}
$$

Como $\omega$ é contínua e limitada em $\Omega$ temos que $g(z)=a(z) \cdot \omega(z)+b(z) \cdot \bar{\omega}(z)$ é limitada em $\Omega$. 
Agora, definamos a função

$$
\begin{aligned}
\tilde{g}: \mathbb{C} & \rightarrow \mathbb{C} \\
z & \mapsto \tilde{g}(z)=\left\{\begin{array}{cl}
g(z) & , \quad z \in \Omega \\
0 & , \quad z \in \mathbb{C} \backslash \Omega
\end{array} .\right.
\end{aligned}
$$

Note que, $\tilde{g}$ é limitada em $\mathbb{C}$ e que $\operatorname{Supp}(\tilde{g}(z)) \subset \Omega \subset \bar{D}_{R}$.

Agora, considere a função $h: \mathbb{C} \rightarrow \mathbb{C}$ dada por

$$
h(z)=-\frac{1}{\pi} \iint_{\Omega} \frac{\tilde{g}(\zeta)}{\zeta-z} d \xi d \eta .
$$

Assim, de $(* 1)$ e pela proposição 2.1, temos que $h \in C^{\alpha}(\mathbb{C})$, com $0<\alpha<1$.

$(\Rightarrow)$ Mostremos que $f$ é analítica em $\Omega$.

Sabemos que $\omega$ é $[a, b]$-pseudo-analítica em $\Omega$, i.e, $\omega \in C^{1}(\Omega)$ e $\frac{\partial \omega}{\partial \bar{z}}=a(z) \omega(z)+b(z) \bar{\omega}(z)$ em $\Omega$, sendo $a(z), b(z) \in C^{\alpha}(\mathbb{C}), 0<\alpha<1$. Logo, $\frac{\partial \omega}{\partial \bar{z}} \in C^{\alpha}(\Omega)$.

Daí, como $\tilde{g}(z)=g(z)=\frac{\partial \omega}{\partial \bar{z}}(z)$, para todo $z \in \Omega$, temos que $\tilde{g}$ é de classe $C^{\alpha}$ em $\Omega$. Consequentemente, pelo por teorema 2.1 , temos que $\frac{\partial h}{\partial z}$ e $\frac{\partial h}{\partial \bar{z}}$ são de classe $C^{\alpha}$ em $\Omega$; além disso, $\frac{\partial h}{\partial \bar{z}}=\tilde{g}(z)=\frac{\partial \omega}{\partial \bar{z}}, \forall z \in \Omega$.

Também, da equação (2.6), temos que $f(z)=\omega(z)-h(z)$; logo,

$$
\frac{\partial f}{\partial \bar{z}}(z)=\frac{\partial \omega}{\partial \bar{z}}(z)-\frac{\partial h}{\partial \bar{z}}(z)=0
$$

para todo $z \in \Omega$.

Portanto, $f$ é analítica em $\Omega$.

$(\Leftarrow)$ Vamos mostrar que $\omega$ dada por $(2.6)$ é $[a, b]$-pseudo-analítica em $\Omega$.

Da equação $(2.6)$ e $(* 2)$, temos

$$
\begin{aligned}
\omega(z) & =f(z)-\frac{1}{\pi} \iint_{\Omega} \frac{\tilde{g}(\zeta)}{\zeta-z} d \xi d \eta \\
& =f(z)+h(z)
\end{aligned}
$$

para todo $z \in \Omega$.

Assim, como $f \in C^{\infty}(\Omega)$ e $h \in C^{\alpha}(\mathbb{C})$, temos que $\omega \in C^{\alpha}(\Omega)$.

Também, como $a, b \in C^{\alpha}(\mathbb{C})$ e $\omega \in C^{\alpha}(\Omega)$, temos que $g(z)=a(z) \omega(z)+b(z) \bar{\omega}(z) \in C^{\alpha}(\Omega)$; 
daí, $\tilde{g} \in C^{\alpha}(\Omega)$.

Assim, pelo teorema 2.1, temos que a função $h$ dada em $(* 2)$ tem derivadas parciais $\frac{\partial h}{\partial z}, \frac{\partial h}{\partial \bar{z}} \in C^{\alpha}(\Omega)$ e $h \in C^{1}(\Omega) ;$ além disso, $\frac{\partial h}{\partial \bar{z}}=\tilde{g}, \forall z \in \Omega$.

Também, como $f \in C^{\infty}(\Omega)$ e $h \in C^{1}(\Omega)$ temos de $(* 3)$ que $\omega \in C^{1}(\Omega)$.

Finalmente, temos que

$$
\begin{aligned}
\frac{\partial \omega}{\partial \bar{z}}(z) & =\frac{\partial f}{\partial \bar{z}}(z)+\frac{\partial h}{\partial \bar{z}}(z)=0+\tilde{g}(z) \\
& =g(z)=a(z) \cdot \omega(z)+b(z) \cdot \bar{\omega}(z)
\end{aligned}
$$

para todo $z \in \Omega$.

Portanto, $\omega$ é $[a, b]$-pseudo-analítica.

O resultado a seguir é uma extensão para funções $[a, b]$-pseudo-analíticas do conhecido resultado de Extensão de Funções Analíticas sobre Singularidades Removíveis.

Teorema 2.2. Seja $\omega(z)$ uma função $[a, b]$-pseudo-analítica e limitada num domínio $0<\left|z-z_{0}\right|<r$. Então, $\omega(z)$ pode ser definido em $z_{0}$ de modo que $\omega(z)$ seja $[a, b]-$ pseudoanalítica em todo o disco $\left|z-z_{0}\right|<r$.

Demonstração.

Seja $D=\left\{z \in \mathbb{C}:\left|z-z_{0}\right|<r\right\}$ e $D_{0}=\left\{z \in \mathbb{C}: 0<\left|z-z_{0}\right|<r\right\}$.

Sabemos que $\omega$ é $[a, b]$-pseudo-analítica em $D_{0}$; logo, pela proposição 2.2 , temos que

$$
f(z)=\omega(z)+\frac{1}{\pi} \iint_{D_{0}} \frac{a(\zeta) \cdot \omega(\zeta)+b(\zeta) \cdot \bar{\omega}(\zeta)}{\zeta-z} d \xi d \eta
$$

é analítica em $D_{0}$.

Por outro lado, como $\omega$ é limitada em $D_{0}$, temos que $f$ é limitada em $D_{0}$; além disso, como $z_{0}$ é uma singularidade isolada para $f$, temos pelo teorema 1.8 que $z_{0}$ é uma singularidade removível de $f$. Assim, existe uma função analítica $\tilde{f}: D \rightarrow \mathbb{C}$ tal que $\left.\tilde{f}\right|_{D_{0}}=f$.

Definamos a função $\tilde{\omega}: D \rightarrow \mathbb{C}$ dada por

$$
\tilde{\omega}(z)=\tilde{f}(z)-\frac{1}{\pi} \iint_{D} \frac{a(\zeta) \cdot \omega(\zeta)+b(\zeta) \cdot \bar{\omega}(\zeta)}{\zeta-z} d \xi d \eta
$$

Considere a função

$$
\begin{aligned}
h: D_{0} & \rightarrow \mathbb{C} \\
z & \mapsto h(z)=a(z) \cdot \omega(z)+b(z) \cdot \bar{\omega}(z)
\end{aligned}
$$


Como $\omega$ é contínua e limitada em $D_{0}$ temos que $h(z)=a(z) \cdot \omega(z)+b(z) \cdot \bar{\omega}(z)$ é limitada em $D_{0}$.

Agora, definamos a função

$$
\begin{aligned}
\tilde{h}: \mathbb{C} & \rightarrow \mathbb{C} \\
z & \mapsto \tilde{h}(z)=\left\{\begin{array}{cl}
h(z), & z \in D_{0} \\
0 & , \quad z \in \mathbb{C} \backslash D_{0}
\end{array} .\right.
\end{aligned}
$$

Note que, $\tilde{h}$ é limitada em $\mathbb{C}$ e que $\operatorname{Supp}(\tilde{h}(z)) \subset D_{0} \subset \bar{D}_{R}$, para algum $R \geq r$.

Considere a função $H: \mathbb{C} \rightarrow \mathbb{C}$ dada por

$$
H(z)=-\frac{1}{\pi} \iint_{D} \frac{\tilde{h}(\zeta)}{\zeta-z} d \xi d \eta
$$

Assim, pela proposição 2.1, temos que $H$ é limitada e $H \in C^{\alpha}(\mathbb{C})$, com $0<\alpha<1$.

Logo, substituindo $(* 2)$ em $(* 1)$ temos

$$
\tilde{\omega}(z)=\tilde{f}(z)-H(z)
$$

Daí, como $\tilde{f}$ é analítica em $D$ e $\tilde{\omega}$ é contínua e limitada em $D$, pela proposição 2.2 , temos que $\tilde{\omega}$ é $[a, b]$-pseudo-analítica em $D$.

\subsection{Princípio da similaridade clássico}

Definição 2.2. Sejam $\omega(z)$ e $f(z)$ duas funções a valores complexos definidas em um domínio limitado $\Omega$. Dizemos que, $f$ e $\omega$ são similares, se existem constantes $M, N>0$ tais que

$N \leq\left|\frac{\omega(z)}{f(z)}\right| \leq M, \forall z \in \Omega$, e além disso, $\frac{\omega}{f} \in C^{0}(\Omega)$.

Agora, vamos provar dois teoremas que afirmam que cada função pseudo-analítica é similar a uma função analítica, e vice-versa.

Teorema 2.3. Seja $\omega: \Omega \rightarrow \mathbb{C}$ uma função $[a, b]$-pseudo-analítica num domínio $\Omega$. Então, existem uma função analítica $f: \Omega \rightarrow \mathbb{C}$ e uma função $s: \bar{\Omega} \rightarrow \mathbb{C}$ Hölder contínua tal que

$$
\omega(z)=e^{s(z)} \cdot f(z)
$$

Além disso, a constante e o expoente de Hölder para s dependem unicamente dos coeficientes $a(z)$ e $b(z)$. 


\section{Demonstração.}

Para $\omega \equiv 0$ o resultado é trivial; de fato, basta tomar $f \equiv 0$ e $s \equiv 0, \forall z \in \Omega$.

Suponhamos $\omega$ não identicamente nula. Definamos $\Omega_{0}=\{z \in \Omega: \omega(z) \neq 0\}$. Assim, como $\omega$ é contínua em $\Omega$ temos que $\Omega_{0}$ é aberto.

Definamos a função $g: \mathbb{C} \rightarrow \mathbb{C}$, sendo

$$
g(z)=\left\{\begin{array}{cl}
a(z)+b(z) \cdot \frac{\bar{\omega}(z)}{\omega(z)} & , \quad z \in \Omega_{0} \\
0 & , \quad z \in \mathbb{C} \backslash \Omega_{0}
\end{array} .\right.
$$

Notemos que:

- $g(z)=0, \forall z \in \mathbb{C} \backslash \Omega_{0}$

- $|g(z)| \leq|a(z)|+|b(z)| \cdot\left|\frac{\bar{\omega}(z)}{\omega(z)}\right|=|a(z)|+|b(z)|, \forall z \in \Omega_{0}$.

Daí, existe $M \geq 0$ tal que $|g(z)| \leq M$, para todo $z \in \mathbb{C}$; isto é, $g$ é limitada em $\mathbb{C}$. Além disso, o $\operatorname{Supp}(g) \subset \bar{D}_{R}$.

Também, definamos

$$
\begin{aligned}
s: \mathbb{C} & \rightarrow \mathbb{C} \\
z & \mapsto s(z)=-\frac{1}{\pi} \iint_{\Omega_{0}} \frac{g(\zeta)}{\zeta-z} d \xi d \eta, \quad \zeta=\xi+i \eta
\end{aligned}
$$

e

$$
\begin{aligned}
f: \Omega & \rightarrow \mathbb{C} \\
z & \mapsto f(z)=e^{-s(z)} \cdot \omega(z)
\end{aligned}
$$

Logo, de $(* 1)$ e pela proposição 2.1 , temos $s \in C^{\alpha}(\mathbb{C})$. Daí, $s \in C^{\alpha}(\bar{\Omega})$.

Por outro lado, sabemos que $\omega(z)$ é $[a, b]$-pseudo-analítica em $\Omega$, i.e, $\omega \in C^{1}(\Omega)$ e

$$
\frac{\partial \omega}{\partial \bar{z}}=a(z) \cdot \omega(z)+b(z) \cdot \bar{\omega}(z)
$$

para todo $z \in \Omega$.

Também, sabemos que $a, b \in C^{\alpha}(\mathbb{C})$ e $\omega(z) \in C^{1}\left(\Omega_{0}\right)$. Daí, $g \in C^{\alpha}\left(\Omega_{0}\right)$. Assim, de $(* 1)$ e pelo teorema 2.1 , temos que a função $s$ tem derivadas parciais Hölder contínuas 
$\frac{\partial s}{\partial \bar{z}}$ e $\frac{\partial s}{\partial z}$ em $\Omega_{0} ;$ além disso, $\frac{\partial s}{\partial \bar{z}}(z)=g(z)=a(z)+b(z) \cdot \frac{\bar{\omega}(z)}{\omega(z)}, \forall z \in \Omega_{0}$ e $s \in C^{1}\left(\Omega_{0}\right)$.

Logo, como $s \in C^{1}\left(\Omega_{0}\right)$ e $\omega \in C^{1}(\Omega)$, temos que $f \in C^{1}\left(\Omega_{0}\right)$. Assim,

$$
\begin{aligned}
& \frac{\partial f}{\partial \bar{z}}(z)=-\frac{\partial s}{\partial \bar{z}}(z) \cdot e^{-s(z)} \cdot \omega(z)+\frac{\partial \omega}{\partial \bar{z}}(z) \cdot e^{-s(z)} \\
& =e^{-s(z)} \cdot\left[-\frac{\partial s}{\partial \bar{z}}(z) \cdot \omega(z)+\frac{\partial \omega}{\partial \bar{z}}(z)\right] \\
& =e^{-s(z)} \cdot\left[\left(-a(z)-b(z) \cdot \frac{\bar{\omega}(z)}{\omega(z)}\right) \cdot \omega(z)+a(z) \cdot \omega(z)+b(z) \cdot \bar{\omega}(z)\right] \\
& =0
\end{aligned}
$$

para todo $z \in \Omega_{0}$.

Portanto, $f$ é analítica em $\Omega_{0}$. Consequentemente, existe $f^{\prime}(z)$, para todo $z \in \Omega_{0}$.

Por outro lado, para todo $z \in \Omega \backslash \Omega_{0}$ temos que $\omega(z)=0$; assim, $f(z)=0, \forall z \in \Omega \backslash \Omega_{0}$; daí, todo $z \in \Omega \backslash \Omega_{0}$ é um zero da função não nula $f$.

Agora, vamos mostrar que $f$ é analítica em $\Omega \backslash \Omega_{0}$. Assim, suponha que:

- Seja $z_{0}$ um ponto isolado qualquer em $\Omega \backslash \Omega_{0}$, isto é, existe $\epsilon>0$ tal que $\overline{B\left(z_{0}, \epsilon\right)} \subset \Omega$ e $B\left(z_{0}, \epsilon\right) \cap\left(\Omega \backslash \Omega_{0}\right)=\left\{z_{0}\right\}$ (ou seja $B\left(z_{0}, \epsilon\right) \backslash\left\{z_{0}\right\} \subset \Omega_{0}$ ). Note que, como $f$ é contínua em $\Omega, f$ é limitada em $\overline{B\left(z_{0}, \epsilon\right)}$; daí, $f$ é limitada em $B\left(z_{0}, \epsilon\right) \backslash\left\{z_{0}\right\}$. Assim, por teorema 1.8 (De Extensão de Riemann), temos que $z_{0}$ é uma singularidade removível de $f$. Logo, pelo teorema 1.6, temos que $f$ é analítica em $z_{0} \in \Omega \backslash \Omega_{0}$.

- Seja $z_{0}$ um ponto não isolado qualquer em $\Omega \backslash \Omega_{0}$, isto é, existe uma seqüência de pontos $\left(z_{j}\right)_{j \in \mathbb{N}}$ em $\Omega \backslash \Omega_{0}$ tal que $\omega\left(z_{j}\right)=0$ e $z_{j} \rightarrow z_{0}, \forall j \in \mathbb{N}$. Assim, pela continuidade de $\omega$, temos que $\omega\left(z_{0}\right)=0$. Agora, tomando uma subsequência se for necessário, podemos assumir que o argumento de $z_{j}-z_{0}$ converge para um ângulo $\theta$.

Como $\omega(z)=u(z)+i v(z) \in C^{1}\left(\Omega \backslash \Omega_{0}\right)$, temos que $u, v \in C^{1}\left(\Omega \backslash \Omega_{0} ; \mathbb{R}\right) ; \log 0 u, v$ são diferenciáveis em qualquer $z_{0} \in \Omega \backslash \Omega_{0}$; assim, pelo teorema do Valor Médio, temos que

$$
\left\{\begin{array}{l}
u\left(z_{j}\right)-u\left(z_{0}\right)=d u\left(\breve{z}_{j}\right) \cdot h=\frac{\partial u}{\partial x}\left(\breve{z}_{j}\right) \cdot \cos \theta_{j}+\frac{\partial u}{\partial y}\left(\breve{z}_{j}\right) \cdot \operatorname{sen} \theta_{j} \\
v\left(z_{j}\right)-v\left(z_{0}\right)=d v\left(\bar{z}_{j}\right) \cdot h=\frac{\partial v}{\partial x}\left(\bar{z}_{j}\right) \cdot \cos \theta_{j}+\frac{\partial v}{\partial y}\left(\bar{z}_{j}\right) \cdot \operatorname{sen} \theta_{j}
\end{array}\right.
$$


$\operatorname{com} z_{j}, z_{0} \in \Omega \backslash \Omega_{0}, h=\left(\cos \theta_{j}, \operatorname{sen} \theta_{j}\right)$ e $\theta_{j}=\arg \left(z_{j}-z_{0}\right)$.

Daí, de $(* 4)$ temos que

$$
\begin{aligned}
u\left(z_{j}\right)-u\left(z_{0}\right)+i\left(v\left(z_{j}\right)-v\left(z_{0}\right)\right)= & \frac{\partial u}{\partial x}\left(\breve{z}_{j}\right) \cdot \cos \theta_{j}+\frac{\partial u}{\partial y}\left(\breve{z}_{j}\right) \cdot \operatorname{sen} \theta_{j}+ \\
& +i\left(\frac{\partial v}{\partial x}\left(\bar{z}_{j}\right) \cdot \cos \theta_{j}+\frac{\partial v}{\partial y}\left(\bar{z}_{j}\right) \cdot \operatorname{sen} \theta_{j}\right) \Rightarrow \\
u\left(z_{j}\right)+i v\left(z_{j}\right)-\left(u\left(z_{0}\right)+i v\left(z_{0}\right)\right)= & \frac{\partial u}{\partial x}\left(\breve{z}_{j}\right) \cdot \cos \theta_{j}+i \frac{\partial v}{\partial x}\left(\bar{z}_{j}\right) \cdot \cos \theta_{j}+ \\
& +\frac{\partial u}{\partial y}\left(\breve{z}_{j}\right) \cdot \operatorname{sen} \theta_{j}+i \frac{\partial v}{\partial y}\left(\bar{z}_{j}\right) \cdot \operatorname{sen} \theta_{j} .
\end{aligned}
$$

Assim,

$\omega\left(z_{j}\right)-\omega\left(z_{0}\right)=\frac{\partial u}{\partial x}\left(\breve{z}_{j}\right) \cdot \cos \theta_{j}+i \frac{\partial v}{\partial x}\left(\bar{z}_{j}\right) \cdot \cos \theta_{j}+\frac{\partial u}{\partial y}\left(\breve{z}_{j}\right) \cdot \operatorname{sen} \theta_{j}+i \frac{\partial v}{\partial y}\left(\bar{z}_{j}\right) \cdot \operatorname{sen} \theta_{j}$.

Logo, como $\breve{z}_{j}, \bar{z}_{j} \rightarrow z_{0}$ e $\theta_{j} \rightarrow \theta$, quando $j \rightarrow \infty$, temos

$$
\begin{aligned}
0 & =\frac{\partial u}{\partial x}\left(z_{0}\right) \cdot \cos \theta+i \frac{\partial v}{\partial x}\left(z_{0}\right) \cdot \cos \theta+\frac{\partial u}{\partial y}\left(z_{0}\right) \cdot \operatorname{sen} \theta+i \frac{\partial v}{\partial y}\left(z_{0}\right) \cdot \operatorname{sen} \theta \\
& =\frac{\partial \omega}{\partial x}\left(z_{0}\right) \cdot \cos \theta+\frac{\partial \omega}{\partial y}\left(z_{0}\right) \cdot \operatorname{sen} \theta
\end{aligned}
$$

Por outro lado, como $\omega\left(z_{0}\right)=0$, em $(* 3)$ temos que

$$
\frac{\partial \omega}{\partial \bar{z}}\left(z_{0}\right)=a\left(z_{0}\right) \cdot \omega\left(z_{0}\right)+b\left(z_{0}\right) \cdot \bar{\omega}\left(z_{0}\right)=a\left(z_{0}\right) \cdot 0+b\left(z_{0}\right) \cdot 0=0 .
$$

Logo,

$$
\begin{aligned}
\frac{\partial \omega}{\partial x}\left(z_{0}\right)+i \frac{\partial \omega}{\partial y}\left(z_{0}\right) & =2 \cdot \frac{\partial \omega}{\partial \bar{z}}\left(z_{0}\right)=0 \\
\frac{\partial \omega}{\partial x}\left(z_{0}\right) & =-i \cdot \frac{\partial \omega}{\partial y}\left(z_{0}\right) .
\end{aligned}
$$

Portanto, substituindo em $(* 5)$, temos que $-i \cdot \frac{\partial \omega}{\partial y}\left(z_{0}\right) \cdot \cos \theta+\frac{\partial \omega}{\partial y}\left(z_{0}\right) \cdot \operatorname{sen} \theta=0$. Assim,

$$
\frac{\partial \omega}{\partial y}\left(z_{0}\right)=0 \quad \text { e } \quad \frac{\partial \omega}{\partial x}\left(z_{0}\right)=0
$$


Daí,

$$
\frac{\partial u}{\partial x}\left(z_{0}\right)=0=\frac{\partial v}{\partial x}\left(z_{0}\right) \quad \text { e } \quad \frac{\partial u}{\partial y}\left(z_{0}\right)=0=\frac{\partial v}{\partial y}\left(z_{0}\right)
$$

Assim, temos que as derivadas parciais $\frac{\partial u}{\partial x}, \frac{\partial u}{\partial y}, \frac{\partial v}{\partial x}, \frac{\partial v}{\partial y}$ satisfazem as equações de Cauchy-Riemann em $z_{0}$. Além disso, como $\omega \in C^{1}\left(\Omega \backslash \Omega_{0}\right)$, temos que $\frac{\partial \omega}{\partial x}$ e $\frac{\partial \omega}{\partial y}$ são contínuas em $z_{0}$. Assim, pelo teorema 1.4, existe

$$
\lim _{z \rightarrow z_{0}} \frac{\omega(z)-\omega\left(z_{0}\right)}{z-z_{0}}=\omega^{\prime}\left(z_{0}\right)
$$

além disso, por $(* 6)$ temos

$$
\omega^{\prime}\left(z_{0}\right)=\frac{\partial \omega}{\partial x}\left(z_{0}\right)=-i \cdot \frac{\partial \omega}{\partial y}\left(z_{0}\right)=0
$$

Assim, em $(* 7)$, temos que

$$
\lim _{z \rightarrow z_{0}} \frac{\omega(z)-\omega\left(z_{0}\right)}{z-z_{0}}=0
$$

Por outro lado, temos

$$
\begin{aligned}
f^{\prime}\left(z_{0}\right) & =\lim _{z \rightarrow z_{0}} \frac{f(z)-f\left(z_{0}\right)}{z-z_{0}}=\lim _{z \rightarrow z_{0}} \frac{e^{-s(z)} \cdot \omega(z)-e^{-s\left(z_{0}\right) \cdot \overbrace{\omega\left(z_{0}\right)}^{=0}}}{z-z_{0}} \\
& =\lim _{z \rightarrow z_{0}}\left\{e^{-s(z)} \cdot \frac{\omega(z)-0}{z-z_{0}}\right\}=\lim _{z \rightarrow z_{0}}\left\{e^{-s(z)} \cdot \frac{\omega(z)-\omega\left(z_{0}\right)}{z-z_{0}}\right\} \\
& =e^{-s\left(z_{0}\right)} \cdot 0=0 .
\end{aligned}
$$

Assim, existe $f^{\prime}\left(z_{0}\right)$ e $f^{\prime}\left(z_{0}\right)=0, \forall z_{0} \in \Omega \backslash \Omega_{0}$. Daí, $f$ é analítica em $\Omega \backslash \Omega_{0}$.

Consequentemente, $f$ é analítica em $\Omega$. Finalmente, como $f$ é analítica e não identicamente nula em $\Omega$, temos que $f$ admite apenas zeros isolados em $\Omega$. Logo, concluímos que $\Omega \backslash \Omega_{0}$ admite apenas pontos isolados. Portanto, podemos escrever

$$
s(z)=-\frac{1}{\pi} \iint_{\Omega}\left[a(\zeta)+b(\zeta) \cdot \frac{\bar{\omega}(\zeta)}{\omega(\zeta)}\right] \frac{d \xi d \eta}{\zeta-z} .
$$


Teorema 2.4. Seja $f: \Omega \rightarrow \mathbb{C}$ uma função analítica definida num domínio limitado $\Omega$. Então, existe uma função $s: \bar{\Omega} \rightarrow \mathbb{C}$ Hölder contínua que se anula em um ponto fixado $z_{0} \in \Omega$ tal que $\omega(z)=e^{s(z)} \cdot f(z) e ́[a, b]$-pseudo-analítica em $\Omega$. Além disso, a constante e o expoente de Hölder para s dependem unicamente dos coeficientes a $(z)$ e b(z).

Demonstração.

Seja $\Omega$ um aberto conexo e limitado.

Para $f \equiv 0$ o resultado é trivial; de fato, basta tomar $s \equiv 0$ e $\omega \equiv 0, \forall z \in \Omega$.

Suponhamos $f$ não identicamente nula. Consideremos $\Omega_{0}=\{z \in \Omega: f(z) \neq 0\} \subset \Omega$. Logo, como $f$ é contínua em $\Omega$, temos que $\Omega_{0}$ é aberto.

Agora, consideremos $\left.f_{0} \equiv f\right|_{\Omega_{0}}$. Note que $f_{0}$ é função analítica em $\Omega_{0}$ e, além disso, $f_{0}(z) \neq 0, \forall z \in \Omega_{0}$.

Primeiro vamos demonstrar o teorema para $f_{0}$ em $\Omega_{0}$. Para isso, considere a seguinte equação

$$
\frac{\partial s}{\partial \bar{z}}(z)=a(z)+b(z) \cdot \frac{\overline{f_{0}}(z)}{f_{0}(z)} \cdot e^{\bar{s}(z)-s(z)},
$$

para todo $z \in \Omega_{0}$.

Suponhamos que existe $s \in C^{1}\left(\Omega_{0}\right)$ tal que $s$ é solução da equação $(* 1)$. Então, definindo $\omega_{0}(z)=e^{s(z)} \cdot f_{0}(z)$, para todo $z \in \Omega_{0}$, teríamos que

$$
\begin{aligned}
\frac{\partial \omega_{0}}{\partial \bar{z}}(z) & =\frac{\partial f_{0}}{\partial \bar{z}}(z) \cdot e^{s(z)}+f_{0}(z) \cdot e^{s(z)} \cdot \frac{\partial s}{\partial \bar{z}}(z) \\
& =f_{0}(z) \cdot e^{s(z)}\left(a(z)+b(z) \cdot \frac{\overline{f_{0}}(z)}{f_{0}(z)} \cdot \frac{e^{\bar{s}(z)}}{e^{s(z)}}\right) \\
& =a(z) \cdot f_{0}(z) \cdot e^{s(z)}+b(z) \cdot \overline{f_{0}}(z) \cdot e^{\bar{s}(z)} \\
& =a(z) \cdot \omega_{0}(z)+b(z) \cdot \overline{\omega_{0}}(z)
\end{aligned}
$$

para todo $z \in \Omega_{0}$. Por conseguinte, teríamos que, $\omega_{0}$ é $[a, b]$-pseudo-analítica em $\Omega_{0}$.

Em resumo, para encontrar $s \in C^{1}\left(\Omega_{0}\right)$ tal que $\omega_{0}(z)=e^{s(z)} \cdot f_{0}(z), \forall z \in \Omega_{0}$ seja solução de $(* 2)$, basta encontrar $s \in C^{1}\left(\Omega_{0}\right)$ tal que $s$ satisfaz $(* 1)$. 
Seja $B=\{s: \Omega \rightarrow \mathbb{C} ; s$ é contínua e limitada $\}$.

Definamos

$$
\begin{aligned}
T: B & \rightarrow B \\
s & \mapsto T(s): \Omega \\
& \rightarrow \mathbb{C} \\
z & \mapsto T s(z)=\sigma(z)-\sigma\left(z_{0}\right)
\end{aligned}
$$

com $z_{0} \in \Omega_{0}$ ponto fixado, sendo $\sigma: \Omega \rightarrow \mathbb{C}$ dada por

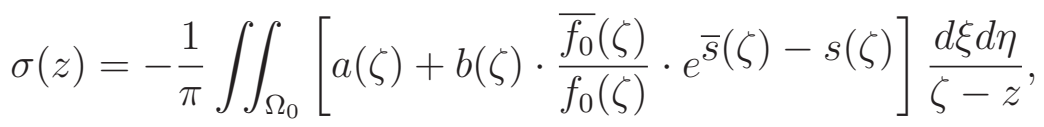

$\operatorname{com} \zeta=\xi+i \eta$

Definamos a função $g: \mathbb{C} \rightarrow \mathbb{C}$, sendo

$$
g(z)=\left\{\begin{array}{cl}
a(z)+b(z) \cdot \frac{\overline{f_{0}}(z)}{f_{0}(z)} \cdot e^{\bar{s}(z)-s(z)} & , \quad z \in \Omega_{0} \\
0 & , \quad z \in \mathbb{C} \backslash \Omega_{0}
\end{array} .\right.
$$

Notemos que:

- $g(z)=0, \forall z \in \mathbb{C} \backslash \Omega_{0}$

- $|g(z)| \leq|a(z)|+|b(z)| \cdot \frac{\left|\overline{f_{0}}(z)\right|}{\left|f_{0}(z)\right|} \cdot \frac{\left|e^{\bar{s}(z)}\right|}{\left|e^{s(z)}\right|}=|a(z)|+|b(z)| \cdot \frac{e^{\operatorname{Re} \bar{s}(z)}}{e^{\operatorname{Re} s(z)}}=|a(z)|+|b(z)|$, $\forall z \in \Omega_{0}$.

Daí, existe $M>0$ tal que $|g(z)| \leq M$, para todo $z \in \mathbb{C}$, isto é, $g$ é limitada em $\mathbb{C}$. Além disso, o $\operatorname{Supp}(g) \subset \bar{D}_{R}$.

Considere a função $\sigma_{1}: \mathbb{C} \rightarrow \mathbb{C}$, sendo

$$
\sigma_{1}(z)=-\frac{1}{\pi} \iint_{\Omega_{0}} \frac{g(\zeta)}{\zeta-z} d \xi d \eta
$$

Daí, pela proposição 2.1, temos que $\sigma_{1}$ satisfaz

$$
\left|\sigma_{1}(z)\right| \leq K M, \forall z \in \Omega
$$

e

$$
\left|\sigma_{1}\left(z_{1}\right)-\sigma_{1}\left(z_{2}\right)\right| \leq K M\left|z_{1}-z_{2}\right|^{\alpha}, \forall z_{1}, z_{2} \in \Omega,
$$

para $0<\alpha<1$ e $K>0$ que depende unicamente de $\alpha$ e $R$. 
Note que, $\sigma=\left.\sigma_{1}\right|_{\Omega} ; \operatorname{logo}, \sigma \in C^{\alpha}(\Omega)$.

Daí, $T s(z)=\sigma(z)-\sigma\left(z_{0}\right)$ é Hölder contínua em $\Omega$.

Por outro lado, sabemos que $B$ é um espaço vetorial real normado, com a norma $\|\varphi\|=\sup _{z \in \Omega}|\varphi(z)|$. Também, pela observação 1.5 , temos que $B$ é um espaço de Banach.

Logo, para $K, M>0$ como acima, definamos:

$$
\Lambda=\left\{\varphi: \Omega \rightarrow \mathbb{C} ;\left|\varphi\left(z_{1}\right)\right| \leq K M \text { e }\left|\varphi\left(z_{1}\right)-\varphi\left(z_{2}\right)\right| \leq K M\left|z_{1}-z_{2}\right|^{\alpha}, \forall z_{1}, z_{2} \in \Omega\right\}
$$

Notemos que:

1. $\Lambda \neq \emptyset$, pois $\varphi \equiv 0 \in \Lambda$.

2. $\Lambda \subset B$, pois dado $h \in \Lambda$, temos que $h$ é limitado em $\Omega$; também, como $h$ é Hölder contínua em $\Omega$, temos que $h$ é contínua em $\Omega$.

3. $\Lambda$ é convexo, isto é, dados $\varphi_{1}, \varphi_{2} \in \Lambda$, temos que $\lambda \cdot \varphi_{1}+(1-\lambda) \varphi_{2} \in \Lambda$, com $\lambda \in[0,1]$.

De fato, como $\varphi_{1}, \varphi_{2} \in \Lambda$, temos que $\left|\varphi_{1}\left(z_{1}\right)\right| \leq K M,\left|\varphi_{2}\left(z_{1}\right)\right| \leq K M, \forall z_{1}, z_{2} \in \Omega$; além disso, $\left|\varphi_{1}\left(z_{1}\right)-\varphi_{1}\left(z_{2}\right)\right| \leq K M\left|z_{1}-z_{2}\right|^{\alpha}$ e $\left|\varphi_{2}\left(z_{1}\right)-\varphi_{2}\left(z_{2}\right)\right| \leq K M\left|z_{1}-z_{2}\right|^{\alpha}$ com $0<\alpha<1$ e $K$ que depende unicamente de $\alpha$ e $R$. Assim, dados $z_{1}, z_{2} \in \Omega$ temos

$$
\begin{aligned}
\left|\left(\lambda \varphi_{1}+(1-\lambda) \varphi_{2}\right)\left(z_{1}\right)\right| & \leq \lambda\left|\varphi_{1}\left(z_{1}\right)\right|+(1-\lambda)\left|\varphi_{2}\left(z_{1}\right)\right| \\
& \leq \lambda K M+(1-\lambda) K M \\
& \leq K M
\end{aligned}
$$

e

$$
\begin{aligned}
\left|\left(\lambda \varphi_{1}+(1-\lambda) \varphi_{2}\right)\left(z_{1}\right)-\left(\lambda \varphi_{1}+(1-\lambda) \varphi_{2}\right)\left(z_{2}\right)\right| \leq & \lambda\left|\left(\varphi_{1}\left(z_{1}\right)-\varphi_{1}\left(z_{2}\right)\right)\right| \\
+ & (1-\lambda)\left|\varphi_{2}\left(z_{1}\right)-\varphi_{2}\left(z_{2}\right)\right| \\
\leq & \lambda K M\left|z_{1}-z_{2}\right|^{\alpha}+ \\
& +(1-\lambda) K M\left|z_{1}-z_{2}\right|^{\alpha} \\
\leq & K M\left|z_{1}-z_{2}\right|^{\alpha} .
\end{aligned}
$$


Portanto, $\Lambda$ é convexo.

4. $\Lambda$ é compacto, isto é, dada $\left(\varphi_{n}\right)_{n \in \mathbb{N}} \subset \Lambda$, existe $\left(\varphi_{n_{j}}\right)_{n_{j} \in \mathbb{N}} \subset\left(\varphi_{n}\right)_{n \in \mathbb{N}}$ tal que $\left(\varphi_{n_{j}}\right)_{n_{j} \in \mathbb{N}}$ converge para alguma função $\varphi \in \Lambda$.

De fato, seja $\left(\varphi_{n}\right)_{n \in \mathbb{N}} \subset \Lambda$, temos:

(a) $\left(\varphi_{n}\right)_{n \in \mathbb{N}}$ é uniformemente limitada, pois $\left|\varphi_{n}(z)\right| \leq K M, \forall n \in \mathbb{N}$ e $z \in \Omega$.

(b) $\left(\varphi_{n}\right)_{n \in \mathbb{N}}$ é eqüicontínuo, isto é, dado $\epsilon>0, \exists \delta(\epsilon)>0$ tal que $\forall z_{1}, z_{2} \in \Omega$, se $\left|z_{1}-z_{2}\right|<\delta$, tem-se que $\left|\varphi_{n}\left(z_{1}\right)-\varphi_{n}\left(z_{2}\right)\right|<\epsilon$, para qualquer $n \in \mathbb{N}$.

De fato, para $n \in \mathbb{N}$ e para quaisquer $z_{1}, z_{2} \in \Omega$, temos que

$$
\begin{aligned}
\left|\varphi_{n}\left(z_{1}\right)-\varphi_{n}\left(z_{2}\right)\right| & \leq K\left|z_{1}-z_{2}\right|^{\alpha} \\
& <K M \delta^{\alpha} .
\end{aligned}
$$

Assim, tomemos $\delta=\left(\frac{\epsilon}{K M}\right)^{1 / \alpha}>0$. Logo, em $(* 5)$ temos que

$$
\left|\varphi_{n}\left(z_{1}\right)-\varphi_{n}\left(z_{2}\right)\right|<\epsilon
$$

para quaisquer $z_{1}, z_{2} \in \Omega$ e qualquer $n \in \mathbb{N}$.

Portanto, de $(a),(b)$ e pelo teorema 1.2 (Ascoli-Arzelá), temos que existe $\left(\varphi_{n_{j}}\right)_{n_{j} \in \mathbb{N}} \subset\left(\varphi_{n}\right)_{n \in \mathbb{N}}$ tal que $\left(\varphi_{n_{j}}\right)_{n_{j} \in \mathbb{N}}$ converge uniformemente a uma função contínua $\varphi \in B$; consequentemente, $\left(\varphi_{n_{j}}\right)_{n_{j} \in \mathbb{N}}$ converge a uma função contínua $\varphi \in B$.

(c) $\varphi \in \Lambda$,

De fato, temos que $\varphi$ satisfaz o seguinte:

- $|\varphi(z)| \leq K M$, para todo $z \in \Omega$, com $K$ que depende unicamente de $\alpha$ e $R$, e $M$ que depende de $a$ e $b$.

De fato, seja $\left(\varphi_{n}\right)_{n \in \mathbb{N}} \in \Lambda$. Como existe $\left(\varphi_{n_{j}}\right)_{n_{j} \in \mathbb{N}} \subset\left(\varphi_{n}\right)_{n \in \mathbb{N}} \subset \Lambda$ tal que $\varphi_{n_{j}} \stackrel{u}{\rightarrow} \varphi$; temos que, dado $\delta>0, \exists n_{j_{0}} \in \mathbb{N}$ tal que se $n_{j}>n_{j_{0}}$ então $\left|\varphi_{n_{j}}(z)-\varphi(z)\right|<\delta, \forall z \in \Omega$.

Assim, $|\varphi(z)| \leq\left|\varphi(z)-\varphi_{n_{j}}(z)\right|+\left|\varphi_{n_{j}}(z)\right| \leq \delta+K M$. 
Daí, fazendo $\delta \rightarrow 0$ temos que

$$
|\varphi(z)| \leq K M
$$

para todo $z \in \Omega$, com $K$ que depende unicamente de $\alpha$ e $R$; e $M$ que depende de $a, b$.

- $\left|\varphi\left(z_{1}\right)-\varphi\left(z_{2}\right)\right| \leq K M\left|z_{1}-z_{2}\right|^{\alpha}$, para todo $z_{1}, z_{2} \in \Omega$, com $K$ que depende unicamente de $\alpha$ e $R$, e $M$ que depende de $a$ e $b$.

De fato,

$$
\begin{aligned}
\left|\varphi\left(z_{1}\right)-\varphi\left(z_{2}\right)\right| \leq & \left|\varphi\left(z_{1}\right)-\varphi_{n_{j}}\left(z_{1}\right)\right|+\left|\varphi_{n_{j}}\left(z_{1}\right)-\varphi_{n_{j}}\left(z_{2}\right)\right|+ \\
& +\left|\varphi_{n_{j}}\left(z_{2}\right)-\varphi\left(z_{2}\right)\right| \\
\leq & \delta+K M\left|z_{1}-z_{2}\right|^{\alpha}+\delta \\
\leq & 2 \delta+K M\left|z_{1}-z_{2}\right|^{\alpha} .
\end{aligned}
$$

Daí, fazendo $\delta \rightarrow 0$ temos que

$$
\left|\varphi\left(z_{1}\right)-\varphi\left(z_{2}\right)\right| \leq K M\left|z_{1}-z_{2}\right|^{\alpha}
$$

para todo $z_{1}, z_{2} \in \Omega$, com $0<\alpha<1, K$ dependendo unicamente de $\alpha$ e $R$, e $M$ que depende de $a$ e $b$.

Portanto, $\varphi \in \Lambda$.

Finalmente, de $(a),(b),(c)$, temos que $\Lambda$ é compacto.

5. $T: B \rightarrow \Lambda$ é contínua em $B$, isto é, para cada $s_{0} \in B$, dado $\epsilon>0$, existe $\delta>0$, tal que se $s \in B$ e $\left\|s-s_{0}\right\|_{u}<\delta$ então $\left\|T s-T s_{0}\right\|_{u}<\epsilon$. 
De fato,

$$
\begin{aligned}
& \left\|T s-T s_{0}\right\|_{u}=\sup _{z \in \Omega}\left|T s(z)-T s_{0}(z)\right| \\
& =\sup _{z \in \Omega} \mid-\frac{1}{\pi} \iint_{\Omega_{0}}\left[b(\zeta) \cdot \frac{\overline{f_{0}}(\zeta)}{f_{0}(\zeta)} \cdot\left(e^{\bar{s}(\zeta)-s(\zeta)}-e^{\overline{s_{0}}(\zeta)-s_{0}(\zeta)}\right)\right] . \\
& \left(\frac{1}{\zeta-z}-\frac{1}{\zeta-z_{0}}\right) d \xi d \eta \\
& \leq \frac{1}{\pi} \sup _{z \in \Omega} \iint_{\Omega_{0}}|b(\zeta)| \cdot\left|\frac{\overline{f_{0}}(\zeta)}{f_{0}(\zeta)}\right| \cdot\left|e^{\bar{s}(\zeta)-s(\zeta)}-e^{\overline{s_{0}}(\zeta)-s_{0}(\zeta)}\right| . \\
& \left|\frac{1}{\zeta-z}-\frac{1}{\zeta-z_{0}}\right| d \xi d \eta \\
& \leq \frac{M_{1}}{\pi} \sup _{z \in \Omega} \iint_{\Omega_{0}}\left|e^{\bar{s}(\zeta)-s(\zeta)}-e^{\overline{s_{0}}(\zeta)-s_{0}(\zeta)}\right| \\
& \left|\frac{1}{\zeta-z}-\frac{1}{\zeta-z_{0}}\right| d \xi d \eta
\end{aligned}
$$

sendo $M_{1}=\sup _{\zeta \in \Omega}|b(\zeta)|>0$.

Agora, vamos mostrar que se $\left|s(\zeta)-s_{0}(\zeta)\right|<\delta$ então

$$
\left|e^{\bar{s}(\zeta)-s(\zeta)}-e^{\overline{s_{0}}(\zeta)-s_{0}(\zeta)}\right|<2 \delta
$$

Assim, para $z \in \Omega$ e $s \in B$ fixados, definamos o caminho

$$
\begin{aligned}
\lambda:[0,1] & \rightarrow \mathbb{C} \\
t & \mapsto \lambda(t)=e^{\alpha_{0}(z)+t\left(\alpha_{1}(z)-\alpha_{0}(z)\right),}
\end{aligned}
$$

sendo

$$
\begin{aligned}
& \alpha_{0}(z)=\overline{s_{0}}(z)-s_{0}(z)=-2 i \operatorname{Im}\left\{s_{0}(z)\right\}, \\
& \alpha_{1}(z)=\bar{s}(z)-s(z)=-2 i \operatorname{Im}\{s(z)\} .
\end{aligned}
$$

Temos que $\lambda(t)$ é um caminho com derivada integrável em $(0,1)$. Além disso, $\lambda(0)=e^{\alpha_{0}(z)}$ e $\lambda(1)=e^{\alpha_{1}(z)}$. 
Note que

$$
\begin{aligned}
\alpha_{0}(z)+t\left(\alpha_{1}(z)-\alpha_{0}(z)\right) & =(t-1) 2 i \operatorname{Im}\left\{s_{0}(z)\right\}-2 i t \operatorname{Im}\{s(z)\} \\
& =\left[2(t-1) \operatorname{Im}\left\{s_{0}(z)\right\}-2 t \operatorname{Im}\{s(z)\}\right] i
\end{aligned}
$$

Logo, $\operatorname{Re}\left\{\alpha_{0}(z)+t\left(\alpha_{1}(z)-\alpha_{0}(z)\right)\right\}=0$.

Também,

$$
\begin{aligned}
\left|\lambda^{\prime}(t)\right| & =\mid e^{\alpha_{0}(z)+t\left(\alpha_{1}(z)-\alpha_{0}(z)\right)|\cdot| \alpha_{1}(z)-\alpha_{0}(z) \mid} \\
& =e^{\operatorname{Re}\left\{\alpha_{0}(z)+t\left(\alpha_{1}(z)-\alpha_{0}(z)\right)\right\}} \cdot\left|\bar{s}(z)-s(z)-\overline{s_{0}}(z)+s_{0}(z)\right| \\
& \leq e^{0} \cdot\left\{\left|\bar{s}(z)-\overline{s_{0}}(z)\right|+\left|s(z)-s_{0}(z)\right|\right\} \\
& \leq\left|\overline{s(z)-s_{0}(z) \mid}+\right| s(z)-s_{0}(z) \mid \\
& <2 \delta .
\end{aligned}
$$

Finalmente, pelo teorema fundamental do cálculo, temos que

$$
\lambda(1)-\lambda(0)=\int_{0}^{1} \lambda^{\prime}(t) d t
$$

Assim,

$$
\begin{aligned}
\mid e^{\bar{s}(\zeta)-s(\zeta)}-e^{\overline{s_{0}}(\zeta)-s_{0}(\zeta) \mid} & =\left|\int_{0}^{1} \lambda^{\prime}(t) d t\right| \leq \int_{0}^{1}\left|\lambda^{\prime}(t)\right| d t \\
& <\int_{0}^{1} 2 \delta d t=2 \delta .
\end{aligned}
$$

Daí, substituindo $(* 7)$ em $(* 6)$ temos

$$
\left\|T s-T s_{0}\right\|_{u}<\frac{2 M_{1} \delta}{\pi} \cdot \sup _{z \in \Omega} \iint_{\Omega_{0}}\left(\frac{1}{|\zeta-z|}+\frac{1}{\left|\zeta-z_{0}\right|}\right) .
$$


Como $\Omega_{0} \subset D_{R}$ temos de $(* 8)$ que

$$
\begin{aligned}
\left\|T s-T s_{0}\right\|_{u} & <\frac{2 M_{1} \delta}{\pi} \cdot \sup _{z \in \Omega}\left\{\int_{0}^{2 \pi} \int_{0}^{2 R} \frac{1}{r_{1}} r_{1} d r_{1} d \theta+\int_{0}^{2 \pi} \int_{0}^{2 R} \frac{1}{r_{2}} r_{2} d r_{2} d \theta\right\} \\
& <\frac{2 M_{1} \delta}{\pi} \cdot \sup _{z \in \Omega}\{4 \pi R+4 \pi R\}=\frac{16 M_{1} R \delta \cdot \pi}{\pi} \\
& <16 M_{1} R \delta .
\end{aligned}
$$

Daí, tomemos $\delta=\frac{\epsilon}{16 M_{1} R}>0$; assim, temos que

$$
\left\|T s-T s_{0}\right\|_{u}<\epsilon
$$

Portanto, $T$ é contínuo em $B$.

Logo, como $T: B \rightarrow \Lambda$ é uma aplicação contínua e $\Lambda \subset B$, temos que a restrição $\left.T\right|_{\Lambda}: \Lambda \rightarrow \Lambda$ é contínua.

Finalmente, como $\Lambda$ é um subconjunto compacto convexo de um espaço de Banach $B$ e $\left.T\right|_{\Lambda}: \Lambda \rightarrow \Lambda$ é uma aplicação contínua, temos pelo teorema 1.3 (Ponto fixo de Shauder) que existe uma função $s \in \Lambda$ tal que $s=\left.T\right|_{\Lambda}(s)$, isto é,

$$
s(z)=-\frac{1}{\pi} \iint_{\Omega_{0}}\left[a(\zeta)+b(\zeta) \cdot \frac{\overline{f_{0}}(\zeta)}{f_{0}(\zeta)} \cdot e^{\bar{s}(\zeta)-s(\zeta)]}\left(\frac{1}{\zeta-z}-\frac{1}{\zeta-z_{0}}\right) d \xi d \eta .\right.
$$

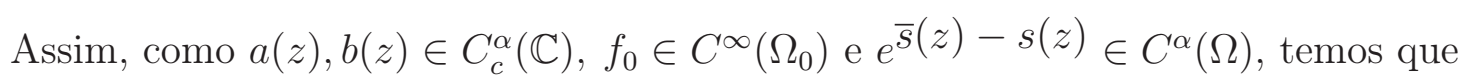

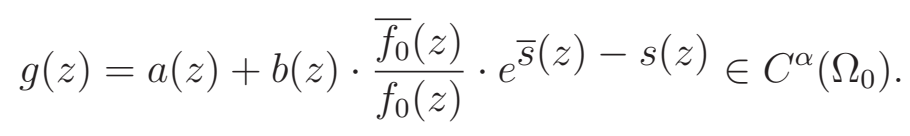

Além disso, sabemos por $(* 3)$ que $g$ é limitada em $\mathbb{C}$ e que $\operatorname{Supp}(g) \subset \bar{D}_{R}$; daí, pelo teorema 2.1, temos que a função $s \in C^{1}\left(\Omega_{0}\right)$ e, além disso,

$$
\frac{\partial s}{\partial \bar{z}}(z)=g(z)=a(z)+b(z) \cdot \frac{\overline{f_{0}}(z)}{f_{0}(z)} \cdot e^{\bar{s}(z)-s(z)}
$$

para todo $z \in \Omega_{0}$. Também, $s$ anula-se em $z_{0} \in \Omega_{0}$.

Logo, de $(* 1)$ temos que $\omega_{0}: \Omega_{0} \rightarrow \mathbb{C}$ dada por

$$
\omega_{0}(z)=e^{s(z)} \cdot f_{0}(z)
$$


é $[a, b]$-pseudo-analítica em $\Omega_{0}$.

Portanto, se $\Omega \backslash \Omega_{0}=\emptyset$, a prova esta completa.

Suponha que $\Omega \backslash \Omega_{0} \neq \emptyset$. Definamos $\omega: \Omega \rightarrow \mathbb{C}$, sendo

$$
\omega(z)=e^{s(z)} \cdot f(z), \forall z \in \Omega
$$

Note que, como $f$ é analítica em $\Omega$, temos que $\Omega \backslash \Omega_{0}$ é discreto. Assim, para cada $z \in \Omega \backslash \Omega_{0}$, existe $r_{z}>0$ tal que $\overline{B\left(z, r_{z}\right)} \subset \Omega$ e $B\left(z, r_{z}\right) \cap\left(\Omega \backslash \Omega_{0}\right)=\{z\} ;$ daí, $B\left(z, r_{z}\right) \backslash\{z\} \subset \Omega_{0}$. Note que, como $\omega$ é contínua em $\Omega$, temos que $\omega$ é limitada em cada $\overline{B\left(z, r_{z}\right)} \backslash\{z\}$. Em particular, $\omega$ é limitada em cada $B\left(z, r_{z}\right) \backslash\{z\}$.

Note que, $\left.\omega\right|_{\Omega_{0}}=\omega_{0}$; logo, $\omega$ é $[a, b]$-pseudo-analítica em $\Omega_{0}$.

Finalmente, como $\omega$ é $[a, b]$-pseudo-analítica em cada $B\left(z, r_{z}\right) \backslash\{z\} \subset \Omega_{0}$ e $\omega$ é limitada em cada $B\left(z, r_{z}\right) \backslash\{z\}$, pelo teorema 2.2 temos que $\omega$ é $[a, b]$-pseudo-analítica em cada $B\left(z, r_{z}\right)$. Portanto, $\omega$ é $[a, b]$-pseudo-analítica em $\Omega$.

Para terminar a demonstração, note que a função

$$
\begin{aligned}
\tilde{s}: \mathbb{C} & \rightarrow \mathbb{C} \\
z & \mapsto \tilde{s}(z)=-\frac{1}{\pi} \iint_{\Omega}\left[a(\zeta)+b(\zeta) \cdot \frac{\overline{f_{0}}(\zeta)}{f_{0}(\zeta)} \cdot e^{\bar{s}(\zeta)-s(\zeta)]} \frac{d \xi d \eta}{\zeta-z}\right.
\end{aligned}
$$

é tal que

- $s=\left.\tilde{s}\right|_{\Omega} ;$

- $|\tilde{s}(z)| \leq K M, \forall z \in \mathbb{C}$

- $\tilde{s} \in C^{\alpha}(\mathbb{C})$.

Logo, basta considerarmos $s=\left.\tilde{s}\right|_{\bar{\Omega}}$. 
Capítulo

3

\section{Princípio da similaridade para campos vetoriais complexos não elíticos}

Seja $\Omega \subset \mathbb{R}^{2}$ um aberto e seja

$$
L=a(x, y) \cdot \frac{\partial}{\partial y}+b(x, y) \cdot \frac{\partial}{\partial x}
$$

um campo vetorial complexo definido em $\Omega$, sendo $a, b \in C^{\infty}(\Omega ; \mathbb{C})$.

Definição 3.1 (Conjunto característico). Definimos o conjunto característico do campo vetorial $L$ como sendo

$$
\operatorname{char}(L)=\{(x, y) \in \Omega: \operatorname{Im}(a \cdot \bar{b})(x, y)=0\}
$$

Definição 3.2 (Campo vetorial elítico). Dizemos que o campo vetorial é elítico em $\Omega$ se $\operatorname{char}(L)=\emptyset$.

Definição 3.3 (Campo vetorial de tipo finito e infinito). Sejam $C^{1}=[L, \bar{L}]$, $C^{2}=\left[L, C^{1}\right], \cdots, C^{n+1}=\left[L, C^{n}\right]$, sendo $[$,$] o colchete de Lie. Dizemos que o campo$ vetorial $L$ é de tipo finito $m$ em $p \in \operatorname{char}(L)$, se $L$ e $C^{j}$ são linearmente dependentes para cada $j<m$, mas $L$ e $C^{m}$ são linearmente independentes.

Se $L$ não é de tipo finito em $p$ então $L$ é de tipo infinito em $p$.

No capítulo anterior, apresentamos resultados sobre o Princípio da similaridade para o campo vetorial complexo $\frac{\partial}{\partial \bar{z}}$, o qual é elítico. Neste Capítulo, vamos apresentar 
resultados sobre Princípio da similaridade para alguns campos vetoriais complexos não elíticos.

Na seção 3.1 os campos vetoriais estudados são de tipo finito, enquanto na seção 3.2 o campo vetorial estudado é de tipo infinito.

\subsection{Princípio da similaridade para alguns campos veto- riais complexos de tipo finito}

Para cada $k \in \mathbb{Z}_{+}$, seja

$$
L_{k}=\frac{\partial}{\partial y}-i(2 k+1) y^{2 k} \frac{\partial}{\partial x}
$$

um campo vetorial complexo definido em $\mathbb{R}^{2}$.

Note que $\operatorname{char}\left(L_{k}\right)=\{(x, 0): x \in \mathbb{R}\} ;$ de fato, $1 \cdot(2 k+1) y^{2 k}=0 \Leftrightarrow y=0$. Portanto, $L_{k}$ é não elítico.

Afirmamos que $L_{k}$ é de tipo finito $2 k$ em $(x, 0) \in \operatorname{char}\left(L_{k}\right)$.

De fato, seja

$$
\bar{L}_{k}=\frac{\partial}{\partial y}+i(2 k+1) y^{2 k} \frac{\partial}{\partial x} .
$$

Assim, temos

$$
\begin{aligned}
C^{1}= & {\left[L_{k}, \bar{L}_{k}\right] } \\
= & L_{k} \cdot \bar{L}_{k}-\bar{L}_{k} \cdot L_{k} \\
= & \left(\frac{\partial}{\partial y}-i(2 k+1) y^{2 k} \frac{\partial}{\partial x}\right) \cdot\left(\frac{\partial}{\partial y}+i(2 k+1) y^{2 k} \frac{\partial}{\partial x}\right)+ \\
& -\left(\frac{\partial}{\partial y}+i(2 k+1) y^{2 k} \frac{\partial}{\partial x}\right) \cdot\left(\frac{\partial}{\partial y}-i(2 k+1) y^{2 k} \frac{\partial}{\partial x}\right) \\
= & 2 i(2 k+1)(2 k) y^{2 k-1} \frac{\partial}{\partial x},
\end{aligned}
$$




$$
\begin{aligned}
& C^{2} \quad=\left[L_{k}, C^{1}\right]=L_{k} \cdot C^{1}-C^{1} \cdot L_{k} \\
& =\left(\frac{\partial}{\partial y}-i(2 k+1) y^{2 k} \frac{\partial}{\partial x}\right) \cdot\left(2 i(2 k+1)(2 k) y^{2 k-1} \frac{\partial}{\partial x}\right)+ \\
& -\left(2 i(2 k+1)(2 k) y^{2 k-1} \frac{\partial}{\partial x}\right) \cdot\left(\frac{\partial}{\partial y}-i(2 k+1) y^{2 k} \frac{\partial}{\partial x}\right) \\
& =2 i(2 k+1)(2 k)(2 k-1) y^{2 k-2} \frac{\partial}{\partial x}, \\
& C^{2 k-1}=\left[L_{k}, C^{2 k-2}\right]=L_{k} \cdot C^{2 k-2}-C^{2 k-2} \cdot L_{k} \\
& =\left(\frac{\partial}{\partial y}-i(2 k+1) y^{2 k} \frac{\partial}{\partial x}\right) \cdot\left(2 i(2 k+1) \cdots(3) y^{2} \frac{\partial}{\partial x}\right)+ \\
& -\left(2 i(2 k+1)(2 k) \cdots(3) y^{2} \frac{\partial}{\partial x}\right) \cdot\left(\frac{\partial}{\partial y}-i(2 k+1) y^{2 k} \frac{\partial}{\partial x}\right) \\
& =2 i(2 k+1)(2 k)(2 k-1) \cdots(2) y \frac{\partial}{\partial x}, \\
& C^{2 k}=\left[L_{k}, C^{2 k-1}\right]=L_{k} \cdot C^{2 k-1}-C^{2 k-1} \cdot L_{k} \\
& =\left(\frac{\partial}{\partial y}-i(2 k+1) y^{2 k} \frac{\partial}{\partial x}\right) \cdot\left(2 i(2 k+1)(2 k)(2 k-1) \cdots(2) y \frac{\partial}{\partial x}\right)+ \\
& -\left(2 i(2 k+1)(2 k)(2 k-1) \cdots(2) y \frac{\partial}{\partial x}\right) \cdot\left(\frac{\partial}{\partial y}-i(2 k+1) y^{2 k} \frac{\partial}{\partial x}\right) \\
& =2 i(2 k+1)(2 k)(2 k-1) \cdots(2)(1) \frac{\partial}{\partial x} .
\end{aligned}
$$

Logo, podemos observar que:

- $L_{k}$ e $C^{j}$ são linearmente dependentes em $(x, 0)$, para cada $j=1, \cdots, 2 k-1$;

- $L_{k}$ e $C^{2 k}$ são linearmente independentes em $(x, 0)$. 
Portanto, $L_{k}$ é de tipo finito $2 k$ em $(x, 0) \in \operatorname{char}\left(L_{k}\right)$.

Como primeiro resultado mostraremos que o Princípio da similaridade vale para o campo $L_{1}$.

Teorema 3.1. Sejam

$$
L_{1}=\frac{\partial}{\partial y}-3 i y^{2} \cdot \frac{\partial}{\partial x}, \quad z_{1}(x, y)=x+i y^{3}
$$

e sejam $A, B \in C^{2+\sigma}\left(\mathbb{R}^{2}\right)$, com $0<\sigma<1$, tais que

$$
B(x, 0)=\frac{\partial B}{\partial y}(x, 0)=0, \forall x \in \mathbb{R} .
$$

Então, existe um conjunto aberto, conexo e limitado $U \subset \mathbb{R}^{2}$ contendo a origem tal que qualquer $\omega \in C^{1}$ solução da equação

$$
L_{1} \omega=A \cdot \omega+B \cdot \bar{\omega} \quad \text { em } U
$$

tem a forma

$$
\omega(x, y)=e^{s(x, y)} \cdot h\left(x+i y^{3}\right),
$$

para alguma função holomorfa $h$ definida em $z_{1}(U)$ e alguma função $s \in C^{\sigma}(U)$.

Reciprocamente, para cada função holomorfa $h$ em $z_{1}(U)$, existe $s \in C^{\sigma}(U)$ tal que a função $\omega(x, y)=e^{s(x, y)} \cdot h\left(x+i y^{3}\right)$ satisfaz a equação (3.4).

Demonstração.

Sejam $A, B \in C^{2+\sigma}\left(\mathbb{R}^{2}\right)$, com $0<\sigma<1$. Fixemos $p=(x, 0) \in \mathbb{R}^{2}$; logo, pela fórmula de Taylor com resto integral temos

$$
\begin{aligned}
A(x, y)= & A(x, 0)+\left(\frac{\partial A}{\partial x}\right)_{p}(x-x)+\left(\frac{\partial A}{\partial y}\right)_{p}(y-0)+\int_{0}^{1}(1-t)\left[\left(\frac{\partial^{2} A}{\partial x^{2}}\right)_{p}(x-x)^{2}+\right. \\
& \left.+\left(\frac{\partial^{2} A}{\partial y \partial x}\right)_{p}(x-x)(y-0)+\frac{\partial^{2} A}{\partial y^{2}}(x, t y) \cdot(y-0)^{2}\right] d t \\
= & A(x, 0)+y \cdot \frac{\partial A}{\partial y}(x, 0)+y^{2} \int_{0}^{1}(1-t) \cdot \frac{\partial^{2} A}{\partial y^{2}}(x, t y) d t \\
= & A_{0}(x)+y A_{1}(x)+y^{2} A_{2}(x, y),
\end{aligned}
$$


sendo $A_{0}(x)=A(x, 0), A_{1}(x)=\frac{\partial A}{\partial y}(x, 0)$ e $A_{2}(x, y)=\int_{0}^{1}(1-t) \cdot \frac{\partial^{2} A}{\partial y^{2}}(x, t y) d t$

Note que $A_{0} \in C^{2+\sigma}(\mathbb{R}), A_{1} \in C^{1+\sigma}(\mathbb{R})$ e $A_{2} \in C^{\sigma}\left(\mathbb{R}^{2}\right)$, com $0<\sigma<1$.

Analogamente, temos

$$
\begin{aligned}
B(x, y)= & B(x, 0)+\left(\frac{\partial B}{\partial x}\right)_{p}(x-x)+\left(\frac{\partial B}{\partial y}\right)_{p}(y-0)+\int_{0}^{1}(1-t)\left[\left(\frac{\partial^{2} B}{\partial x^{2}}\right)_{p}(x-x)^{2}+\right. \\
& \left.+\left(\frac{\partial^{2} B}{\partial y \partial x}\right)_{p}(x-x)(y-0)+\frac{\partial^{2} B}{\partial y^{2}}(x, t y) \cdot(y-0)^{2}\right] d t \\
= & B(x, 0)+y \cdot \frac{\partial B}{\partial y}(x, 0)+y^{2} \int_{0}^{1}(1-t) \cdot \frac{\partial^{2} B}{\partial y^{2}}(x, t y) d t \\
= & 0+y \cdot 0+y^{2} B_{2}(x, y) \\
= & y^{2} B_{2}(x, y),
\end{aligned}
$$

sendo $B_{2}(x, y)=\int_{0}^{1}(1-t) \cdot \frac{\partial^{2} B}{\partial y^{2}}(x, t y) d t ;$ daí, $B_{2} \in C^{\sigma}\left(\mathbb{R}^{2}\right)$, com $0<\sigma<1$.

Assim, temos

$$
\begin{aligned}
L_{1} \omega & =\left[A_{0}(x)+y A_{1}(x)+y^{2} A_{2}(x, y)\right] \cdot \omega+y^{2} B_{2}(x, y) \cdot \bar{\omega} \\
& =y^{2} A_{2}(x, y) \cdot \omega+y^{2} B_{2}(x, y) \cdot \bar{\omega}+\left[A_{0}(x)+y A_{1}(x)\right] \cdot \omega .
\end{aligned}
$$

Agora, vamos transformar $(* 1)$ numa equação da forma

$$
L_{1} \mu=y^{2} \cdot P(x, y) \cdot \mu+y^{2} \cdot Q(x, y) \cdot \bar{\mu}
$$

sendo $P, Q \in C^{\sigma}$ numa vizinhança de $(0,0)$.

Para isso, considere uma vizinhança $U_{\epsilon, \delta}=(-\epsilon, \epsilon) \times(-\delta, \delta) \subset \mathbb{R}^{2}$ e vamos procurar $\omega$ na forma $\omega=\mu \cdot \nu$, com $\mu \in C^{1}\left(U_{\epsilon, \delta}\right), \nu \in C^{1+\sigma}\left(U_{\epsilon, \delta}\right)$ e $\nu \neq 0$. Assim, temos em (*1) o seguinte:

$$
L_{1}(\mu \cdot \nu)=y^{2} \cdot A_{2}(x, y) \cdot \mu \cdot \nu+y^{2} \cdot B_{2}(x, y) \cdot \overline{\mu \cdot \nu}+\left[A_{0}(x)+y A_{1}(x)\right] \cdot \mu \cdot \nu,
$$

para todo $(x, y) \in U_{\epsilon, \delta}$. 
Logo, pela regra de Leibniz, temos

$$
\left(L_{1} \mu\right) \cdot \nu+\mu \cdot\left(L_{1} \nu\right)=y^{2} \cdot A_{2}(x, y) \cdot \mu \cdot \nu+y^{2} \cdot B_{2}(x, y) \cdot \bar{\mu} \cdot \bar{\nu}+\left[A_{0}(x)+y A_{1}(x)\right] \cdot \mu \cdot \nu
$$

para todo $(x, y) \in U_{\epsilon, \delta}$.

Daí,

$$
\begin{aligned}
L_{1} \mu & =-\frac{\mu}{\nu} \cdot L_{1} \nu+y^{2} \cdot A_{2}(x, y) \cdot \mu+y^{2} \cdot B_{2}(x, y) \cdot \bar{\mu} \cdot \frac{\bar{\nu}}{\nu}+\left[A_{0}(x)+y A_{1}(x)\right] \cdot \mu \\
& =y^{2} \cdot A_{2}(x, y) \cdot \mu+y^{2} \cdot \tilde{B}_{2}(x, y) \cdot \bar{\mu}+\left[A_{0}(x)+y \cdot A_{1}(x)-\frac{1}{\nu} \cdot L_{1} \nu\right] \cdot \mu \\
& =y^{2} \cdot A_{2}(x, y) \cdot \mu+y^{2} \cdot \tilde{B}_{2}(x, y) \cdot \bar{\mu}+\left[\frac{\left(A_{0}(x)+y A_{1}(x)\right) \nu-L_{1} \nu}{\nu}\right] \cdot \mu,
\end{aligned}
$$

para todo $(x, y) \in U_{\epsilon, \delta}$, sendo $\tilde{B}_{2}=B_{2} \cdot \frac{\bar{\nu}}{\nu} \in C^{\sigma}\left(U_{\epsilon, \delta}\right)$.

Logo, basta encontrar $\nu$, com $\nu \neq 0$, que satisfaça

$$
L_{1} \nu(x, y)-\left(A_{0}(x)+y A_{1}(x)\right) \cdot \nu(x, y)=y^{2} \cdot g(x, y)
$$

para todo $(x, y) \in U_{\epsilon, \delta}$ e para alguma $g \in C^{\sigma}\left(U_{\epsilon, \delta}\right)$.

Para isso, vamos procurar $\nu$ na forma

$$
\nu(x, y)=1+\alpha_{1}(x) \cdot y+\alpha_{2}(x) \cdot y^{2}
$$

$\operatorname{com} \alpha_{1} \in C^{2+\alpha}((-\epsilon, \epsilon))$ e $\alpha_{2} \in C^{1+\alpha}((-\epsilon, \epsilon))$, para todo $(x, y) \in U_{\epsilon, \delta}$.

Observe que para $\nu$ da forma $(* 4)$ tem-se $\nu(0,0)=1$; logo, para $\epsilon, \delta>0$ suficientemente pequenos temos $\nu(x, y)>0, \forall(x, y) \in U_{\epsilon, \delta}$.

Note que:

$$
\begin{aligned}
\text { - } L_{1} \nu(x, y)=\alpha_{1}(x)+2 y \alpha_{2}(x)- & 3 i y^{3} \alpha_{1}{ }^{\prime}(x)-3 i y^{4} \alpha_{2}{ }^{\prime}(x) \\
\bullet & {\left[A_{0}(x)+y A_{1}(x)\right] \cdot \nu(x, y)=} \\
& A_{0}(x)+A_{0}(x) \alpha_{1}(x) \cdot y+A_{0}(x) \alpha_{2}(x) \cdot y^{2}+ \\
& +A_{1}(x) \cdot y+A_{1}(x) \alpha_{1}(x) \cdot y^{2}+A_{1}(x) \alpha_{2}(x) \cdot y^{3} \\
= & A_{0}(x)+\left[A_{0}(x) \alpha_{1}(x)+A_{1}(x)\right] y+\left[A_{0}(x) \alpha_{2}(x)+\right. \\
& \left.+A_{1}(x) \alpha_{1}(x)\right] y^{2}+A_{1}(x) \alpha_{2}(x) \cdot y^{3}
\end{aligned}
$$


para todo $(x, y) \in U_{\epsilon, \delta}$.

Logo, temos

$$
\begin{aligned}
L_{1} \nu(x, y)-\left[A_{0}(x)+y A_{1}(x)\right] \cdot \nu(x, y)= & {\left[\alpha_{1}(x)-A_{0}(x)\right]+\left[2 \alpha_{2}(x)-A_{0}(x) \alpha_{1}(x)+\right.} \\
& \left.-A_{1}(x)\right] y-\left[3 i y \alpha_{1}{ }^{\prime}(x)+3 i y^{2} \alpha_{2}{ }^{\prime}(x)+\right. \\
& \left.+A_{0}(x) \alpha_{2}(x)+A_{1}(x) \alpha_{1}(x)+A_{1}(x) \alpha_{2}(x) y\right] y^{2},
\end{aligned}
$$

para todo $(x, y) \in U_{\epsilon, \delta}$. Consequentemente, de $(* 3)$ temos o seguinte sistema de equações

$$
\left\{\begin{array}{ll}
\alpha_{1}(x)-A_{0}(x) & =0 \\
2 \alpha_{2}(x)-A_{0}(x) \alpha_{1}(x)-A_{1}(x) & =0 \\
-3 i y \alpha_{1}{ }^{\prime}(x)-3 i y^{2} \alpha_{2}{ }^{\prime}(x)-A_{0}(x) \alpha_{2}(x)-A_{1}(x) \alpha_{1}(x)-A_{1}(x) \alpha_{2}(x) y & =g(x, y)
\end{array},(* 5)\right.
$$

para todo $(x, y) \in U_{\epsilon, \delta}$.

Logo,

$$
\begin{aligned}
\alpha_{1}(x)= & A_{0}(x) \in C^{2+\sigma}((-\epsilon, \epsilon)), \\
\alpha_{2}(x)= & \frac{1}{2}\left[A_{0}^{2}(x)+A_{1}(x)\right] \in C^{1+\sigma}((-\epsilon, \epsilon)), \\
g(x, y)= & -\left[\frac{3}{2} A_{0}(x) A_{1}(x)+\frac{1}{2} A_{1}(x) A_{0}^{2}(x)+\frac{1}{2} A_{1}^{2}(x)+3 i y A_{0}^{\prime}(x)+\right. \\
& \left.+3 i y^{2} A_{0}(x) A_{0}^{\prime}(x)+\frac{3}{2} i y^{2} A_{1}^{\prime}(x)+\frac{1}{2} A_{0}^{3}(x)\right] \in C^{\sigma}\left(U_{\epsilon, \delta}\right) .
\end{aligned}
$$

Portanto,

$$
\nu(x, y)=1+A_{0}(x) \cdot y+\frac{1}{2}\left[A_{0}^{2}(x)+A_{1}(x)\right] y^{2},
$$

para todo $(x, y) \in U_{\epsilon, \delta}$; também, $\nu \in C^{1+\sigma}\left(U_{\epsilon, \delta}\right)$ e satisfaz as propriedades requeridas.

Finalmente, usando a $\nu$ obtida acima em $(* 2)$ temos

$$
\begin{aligned}
L_{1} \mu & =y^{2} \cdot A_{2}(x, y) \cdot \mu+y^{2} \cdot \tilde{B}_{2}(x, y) \cdot \bar{\mu}-y^{2} \cdot \frac{1}{\nu} \cdot g(x, y) \cdot \mu \\
& =y^{2}\left[A_{2}(x, y)-\frac{1}{\nu} \cdot g(x, y)\right] \cdot \mu+y^{2} \cdot \tilde{B}_{2}(x, y) \cdot \bar{\mu} \\
& =y^{2}\left[\frac{A_{2}(x, y) \cdot \nu(x, y)-g(x, y)}{\nu(x, y)}\right] \cdot \mu+y^{2} \cdot \tilde{B}_{2}(x, y) \cdot \bar{\mu}
\end{aligned}
$$


Considerando $P(x, y)=\frac{A_{2}(x, y) \cdot \nu(x, y)-g(x, y)}{\nu(x, y)} \quad$ e $\quad Q(x, y)=\tilde{B}_{2}(x, y)$, temos a equação

$$
L_{1} \mu=y^{2} \cdot P(x, y) \cdot \mu+y^{2} \cdot Q(x, y) \cdot \bar{\mu}
$$

sendo $P, Q \in C^{\sigma}\left(U_{\epsilon, \delta}\right)$.

Portanto, encontrar $\omega \in C^{1}\left(U_{\epsilon, \delta}\right)$ tal que $\omega$ seja solução da equação (3.4) é equivalente a encontrar $\mu \in C^{1}\left(U_{\epsilon, \delta}\right)$ tal que $\mu$ satisfaça a equação $(* 6)$.

Considere a função

$$
\begin{aligned}
z_{1}: U_{\epsilon, \delta} & \rightarrow z_{1}\left(U_{\epsilon, \delta}\right) \subset \mathbb{C} \\
(x, y) & \mapsto z_{1}(x, y)=x+i y^{3} .
\end{aligned}
$$

Notemos que $z_{1}\left(U_{\epsilon, \delta}\right)=\left\{x+i y^{3}:|x| \leq \epsilon,|y| \leq \delta\right\}$ e que $0 \in z_{1}\left(U_{\epsilon, \delta}\right)$.

Por outro lado, para todo $(x, y) \in U_{\epsilon, \delta}$ temos que:

$$
\begin{aligned}
& -L_{1}\left(\bar{z}_{1}(x, y)\right)=L_{1}\left(x-i y^{3}\right)=\frac{\partial}{\partial y}\left(x-i y^{3}\right)-3 i y^{2} \frac{\partial}{\partial x}\left(x-i y^{3}\right)=-6 i y^{2} \\
& \text { - } L_{1}\left(z_{1}(x, y)\right)=L_{1}\left(x+i y^{3}\right)=\frac{\partial}{\partial y}\left(x+i y^{3}\right)-3 i y^{2} \frac{\partial}{\partial x}\left(x+i y^{3}\right)=0 .
\end{aligned}
$$

Logo, o push-forward de $L_{1} \mu$ pela função $z_{1}$ é definido por $z_{1 *}\left(L_{1} \mu\right)=\left(L_{1} \mu\right) \circ z_{1}^{-1}$, para qualquer $L_{1} \mu \in C^{\infty}\left(U_{\epsilon, \delta}\right)$.

Assim, em $(* 6)$ temos

$$
\begin{aligned}
\left(L_{1} \mu\right) \circ z_{1}^{-1} & =\left(y^{2} \cdot P \cdot \mu\right) \circ z_{1}^{-1}+\left(y^{2} \cdot Q \cdot \bar{\mu}\right) \circ z_{1}^{-1} \\
& =\left[\left(y^{2} \cdot P\right) \circ z_{1}^{-1}\right] \cdot\left[\mu \circ z_{1}^{-1}\right]+\left[\left(y^{2} \cdot Q\right) \circ z_{1}^{-1}\right] \cdot\left[\bar{\mu} \circ z_{1}^{-1}\right]
\end{aligned}
$$

Agora, considerando os pontos de $z_{1}\left(U_{\epsilon, \delta}\right)$ escritos na forma $z_{1}=\xi+i \eta$ temos

$$
\begin{gathered}
\xi=\frac{z_{1}+\bar{z}_{1}}{2} \text { e } \eta=\frac{z_{1}-\bar{z}_{1}}{2 i} \\
\frac{\partial}{\partial \xi}=\frac{\partial}{\partial z_{1}}+\frac{\partial}{\partial \bar{z}_{1}} \text { e } \frac{\partial}{\partial \eta}=i\left(\frac{\partial}{\partial z_{1}}-\frac{\partial}{\partial \bar{z}_{1}}\right) .
\end{gathered}
$$

Considere $\tilde{L}_{1}: C^{\infty}\left(z_{1}\left(U_{\epsilon, \delta}\right)\right) \rightarrow C^{\infty}\left(z_{1}\left(U_{\epsilon, \delta}\right)\right)$ o campo vetorial da mudança de coordenadas 
definido por

$$
\begin{aligned}
\tilde{L}_{1} & =L_{1} \xi\left(z_{1}^{-1}(\xi, \eta)\right) \cdot \frac{\partial}{\partial \xi}+L_{1} \eta\left(z_{1}^{-1}(\xi, \eta)\right) \cdot \frac{\partial}{\partial \eta} \\
& =L_{1} \xi\left(z_{1}^{-1}\left(x, y^{3}\right)\right) \cdot \frac{\partial}{\partial \xi}+L_{1} \eta\left(z_{1}^{-1}\left(x, y^{3}\right)\right) \cdot \frac{\partial}{\partial \eta} \\
& =L_{1} \xi(x, y) \cdot \frac{\partial}{\partial \xi}+L_{1} \eta(x, y) \cdot \frac{\partial}{\partial \eta} \\
& =\left(L_{1}\left(\frac{z_{1}+\bar{z}_{1}}{2}\right)\right)(x, y) \cdot\left[\frac{\partial}{\partial z_{1}}+\frac{\partial}{\partial \bar{z}_{1}}\right]+\left(L_{1}\left(\frac{z_{1}-\bar{z}_{1}}{2 i}\right)\right)(x, y) \cdot\left[\frac{\partial}{\partial z_{1}}-\frac{\partial}{\partial \bar{z}_{1}}\right] i \\
& =\frac{1}{2}\left(L_{1} z_{1}+L_{1} \bar{z}_{1}\right)(x, y) \cdot\left[\frac{\partial}{\partial z_{1}}+\frac{\partial}{\partial \bar{z}_{1}}\right]+\frac{1}{2}\left(L_{1} z_{1}-L_{1} \bar{z}_{1}\right)(x, y) \cdot\left[\frac{\partial}{\partial z_{1}}-\frac{\partial}{\partial \bar{z}_{1}}\right] \\
& =L_{1} z_{1}(x, y) \cdot \frac{\partial}{\partial z_{1}}+L_{1} \bar{z}_{1}(x, y) \cdot \frac{\partial}{\partial \bar{z}_{1}},
\end{aligned}
$$

para todo $(x, y) \in U_{\epsilon, \delta}$.

Logo,

$$
\tilde{L}_{1}\left(\mu \circ z_{1}^{-1}\right)=L_{1} z_{1}(x, y) \cdot \frac{\partial}{\partial z_{1}}\left(\mu \circ z_{1}^{-1}\right)+L_{1} \bar{z}_{1}(x, y) \cdot \frac{\partial}{\partial \bar{z}_{1}}\left(\mu \circ z_{1}^{-1}\right)
$$

para todo $(x, y) \in U_{\epsilon, \delta}$.

Por outro lado, pela proposição 1.3 temos que $\tilde{L}_{1}\left(\mu \circ z_{1}^{-1}\right)=\left(L_{1} \mu\right) \circ z_{1}^{-1}$. Assim, de $(* 10)$ e $(* 9)$ temos

$$
\begin{aligned}
L_{1} z_{1}(x, y) \frac{\partial}{\partial z_{1}}\left(\mu \circ z_{1}^{-1}\right)+L_{1} \bar{z}_{1}(x, y) \frac{\partial}{\partial \bar{z}_{1}}\left(\mu \circ z_{1}^{-1}\right)= & {\left[\left(y^{2} \cdot P\right) \circ z_{1}^{-1}\right] \cdot\left[\mu \circ z_{1}^{-1}\right]+} \\
& +\left[\left(y^{2} \cdot Q\right) \circ z_{1}^{-1}\right] \cdot\left[\bar{\mu} \circ z_{1}^{-1}\right]
\end{aligned}
$$

para todo $(x, y) \in U_{\epsilon, \delta}$.

Logo, considere $\psi=\mu \circ z_{1}^{-1}: z_{1}\left(U_{\epsilon, \delta}\right) \rightarrow \mathbb{C}$ e $\bar{\psi}=\bar{\mu} \circ z_{1}^{-1}: z_{1}\left(U_{\epsilon, \delta}\right) \rightarrow \mathbb{C}$. Como $z_{1} \in C^{\infty}\left(U_{\epsilon, \delta}\right)$ e $\mu \in C^{1}\left(U_{\epsilon, \delta}\right)$ temos que $\psi \in C^{1}\left(z_{1}\left(U_{\epsilon, \delta}\right)\right)$. Logo, substituindo em $(* 11)$ 
temos

$$
\underbrace{L_{1} z_{1}(x, y)}_{=0} \cdot \frac{\partial \psi}{\partial z_{1}}+L_{1} \bar{z}_{1}(x, y) \cdot \frac{\partial \psi}{\partial \bar{z}_{1}}=y^{2} \cdot P\left(z_{1}^{-1}(\xi, \eta)\right) \cdot \psi+y^{2} \cdot Q\left(z_{1}^{-1}(\xi, \eta)\right) \cdot \bar{\psi}
$$

o que implica

$$
-6 i y^{2} \cdot \frac{\partial \psi}{\partial \bar{z}_{1}}=y^{2} \cdot P(\xi, \sqrt[3]{\eta}) \cdot \psi+y^{2} \cdot Q(\xi, \sqrt[3]{\eta}) \cdot \bar{\psi}
$$

daí,

$$
\frac{\partial \psi}{\partial \bar{z}_{1}}=\frac{i}{6}[P(\xi, \sqrt[3]{\eta}) \cdot \psi+Q(\xi, \sqrt[3]{\eta}) \cdot \bar{\psi}]
$$

Sabemos que, $\psi \in C^{1}\left(z_{1}\left(U_{\epsilon, \delta}\right)\right)$ e $P \circ z_{1}^{-1}, Q \circ z_{1}^{-1} \in C^{\sigma}\left(z_{1}\left(U_{\epsilon, \delta}\right)\right)$.

Agora, considere a função $\phi: \mathbb{C} \rightarrow \mathbb{C}$ tais que:

- $\phi \in C_{c}^{\infty}(\mathbb{C})$

- $\operatorname{Supp}(\phi) \subset z_{1}\left(U_{\epsilon, \delta}\right)$;

- $\phi \equiv 1$ num domínio compacto $\bar{V}$ de $(0,0), \operatorname{com} \bar{V} \subset z_{1}\left(U_{\epsilon, \delta}\right)$.

Assim, considere em $z_{1}\left(U_{\epsilon, \delta}\right)$ a seguinte equação

$$
\frac{\partial \vartheta}{\partial \bar{z}_{1}}=\frac{i}{6}[\tilde{P}(\xi, \eta) \cdot \vartheta+\tilde{Q}(\xi, \eta) \cdot \bar{\vartheta}]
$$

sendo $\tilde{P}, \tilde{Q}: \mathbb{C} \rightarrow \mathbb{C}$ tais que:

$$
\begin{aligned}
& \tilde{P}(\xi, \eta)=\left\{\begin{array}{cl}
\left(P \circ z_{1}^{-1}\right)(\xi, \eta) \cdot \phi(\xi, \eta) & , \quad(\xi, \eta) \in z_{1}\left(U_{\epsilon, \delta}\right) \\
0 & , \quad(\xi, \eta) \in \mathbb{C} \backslash z_{1}\left(U_{\epsilon, \delta}\right)
\end{array},\right. \\
& \tilde{Q}(\xi, \eta)=\left\{\begin{array}{cl}
\left(Q \circ z_{1}^{-1}\right)(\xi, \eta) \cdot \phi(\xi, \eta) & , \quad(\xi, \eta) \in z_{1}\left(U_{\epsilon, \delta}\right) \\
0 & , \quad(\xi, \eta) \in \mathbb{C} \backslash z_{1}\left(U_{\epsilon, \delta}\right)
\end{array} .\right.
\end{aligned}
$$

Note que $\tilde{P}, \tilde{Q} \in C^{\sigma}(\mathbb{C})$.

Assim, como $\psi$ satisfaz $(* 13)$, para todo $(\xi, \eta) \in V$, então $\psi$ é uma função $[\tilde{P}, \tilde{Q}]-$ pseudoanalítica em $V$. Logo, pelo teorema 2.3 (Princípio da similaridade clássico), temos que existe uma função analítica $h$ no interior de $V$ e $s_{0} \in C^{\sigma}(\bar{V})$ tal que

$$
\psi(\xi, \eta)=e^{s_{0}(\xi, \eta)} \cdot h(\xi, \eta)
$$

para todo $(\xi, \eta) \in V$. 
Finalmente, compondo novamente temos $\left(\psi \circ z_{1}\right)=e^{s_{0} \circ z_{1}} \cdot\left(h \circ z_{1}\right)$.

Assim, dado $(x, y) \in U=z_{1}^{-1}(V)$, temos

$$
\left(\psi \circ z_{1}\right)(x, y)=e^{\left(s_{0} \circ z_{1}\right)(x, y)} \cdot\left(h \circ z_{1}\right)(x, y)
$$

isto é,

$$
\mu(x, y)=e^{\left(s_{0} \circ z_{1}\right)(x, y)} \cdot h\left(z_{1}(x, y)\right) .
$$

Logo, temos que $(* 15)$ é solução da equação $(* 6)$. Mas, sabemos que $\mu=\frac{\omega}{\nu}, \operatorname{com} \nu \neq 0$.

Assim, em $(* 15)$, temos

$$
\frac{\omega(x, y)}{\nu(x, y)}=e^{\left(s_{0} \circ z_{1}\right)(x, y)} \cdot h\left(x+i y^{3}\right)
$$

o que implica

$$
\omega(x, y)=\nu(x, y) \cdot e^{\left(s_{0} \circ z_{1}\right)(x, y)} \cdot h\left(x+i y^{3}\right)
$$

$\log \mathrm{O}$

$$
\omega(x, y)=e^{\left(s_{0} \circ z_{1}\right)(x, y)+\log \nu(x, y)} \cdot h\left(x+i y^{3}\right),
$$

para todo $(x, y) \in U=z_{1}^{-1}(V)$.

Portanto, de $(* 16)$ temos que $\omega$ tem a forma desejada.

Reciprocamente, com a mesma notação que acima, seja $h$ uma função holomorfa em $V \subset z_{1}\left(U_{\epsilon, \delta}\right)$. Logo, pelo teorema 2.4 (Princípio da similaridade clássico), temos que existe uma função $s_{0} \in C^{\sigma}(\bar{V})$ tal que $\psi(\xi, \eta)=e^{s_{0}(\xi, \eta)} \cdot h(\xi, \eta)$ é $[\tilde{P}, \tilde{Q}]-$ pseudo-analítica em $V$, isto é, $\psi \in C^{1}(V)$ e satisfaz a equação $(* 13)$. Logo, como $\psi=\mu \circ z_{1}^{-1}$, segue que a função dada por (* 16) satisfaz (3.4), para todo $(x, y) \in U=z_{1}^{-1}(V)$.

A seguir, vamos estender os argumentos utilizados acima para verificar que o Princípio da similaridade é válido para o campo vetorial complexo de tipo finito $L_{k}$, com $k \in \mathbb{Z}_{+}$. 
Teorema 3.2. Sejam

$$
L_{k}=\frac{\partial}{\partial y}-i(2 k+1) y^{2 k} \cdot \frac{\partial}{\partial x}, \quad z_{k}=x+i y^{2 k+1}
$$

e $A, B \in C^{2 k+\sigma}\left(\mathbb{R}^{2}\right), 0<\sigma<1$, tais que

$$
B(x, 0)=\frac{\partial B}{\partial y}(x, 0)=\cdots=\frac{\partial^{2 k-1} B}{\partial^{2 k-1} y}(x, 0)=0, \forall x \in \mathbb{R}
$$

Então, existe um conjunto aberto, conexo e limitado $U \subset \mathbb{R}^{2}$ contendo a origem tal que qualquer $\omega \in C^{1}$ solução da equação

$$
L_{k} \omega=A \cdot \omega+B \cdot \bar{\omega} \quad \text { em } U
$$

tem a forma

$$
\omega(x, y)=e^{s(x, y)} \cdot h\left(x+i y^{2 k+1}\right)
$$

para alguma função holomorfa $h$ definida em $z_{k}(U)$ e alguma função $s \in C^{\sigma}(U)$.

Reciprocamente, para cada função holomorfa $h$ em $z_{k}(U)$, existe $s \in C^{\sigma}(U)$ tal que a função $\omega(x, y)=e^{s(x, y)} \cdot h\left(x+i y^{2 k+1}\right)$ satisfaz a equação (3.8).

Demonstração.

Sejam $A, B \in C^{2 k+\sigma}\left(\mathbb{R}^{2}\right)$, com $0<\sigma<1$. Fixemos $p=(x, 0) \in \mathbb{R}^{2}$; logo, pela fórmula de Taylor com resto integral temos

$$
A(x, y)=\sum_{n=0}^{2 k-1} \frac{1}{n !} D^{n} A(x, 0)+\frac{1}{(2 k-1) !} \int_{0}^{1}(1-t)^{2 k-1} D^{2 k} A(x, t y) d t
$$

com

$$
\begin{aligned}
D^{n} & =y^{n} \frac{\partial^{n}}{\partial y^{n}}+\sum_{j=1}^{n}\left(\begin{array}{l}
n \\
j
\end{array}\right) \underbrace{(x-x)^{j}}_{=0} \cdot(y-0)^{n-j} \frac{\partial^{n}}{\partial x^{j} \partial y^{n-j}} \\
& =y^{n} \cdot \frac{\partial^{n}}{\partial y^{n}} .
\end{aligned}
$$


Daí, temos

$$
\begin{aligned}
A(x, y)= & \sum_{n=0}^{2 k-1} \frac{1}{n !} \cdot y^{n} \cdot \frac{\partial^{n} A}{\partial y^{n}}(x, 0)+\frac{1}{(2 k-1) !} \int_{0}^{1}(1-t)^{2 k-1} \cdot y^{2 k} \cdot \frac{\partial^{2 k} A}{\partial y^{2 k}}(x, t y) d t \\
= & A(x, 0)+y \cdot \frac{\partial A}{\partial y}(x, 0)+\frac{1}{2 !} \cdot y^{2} \cdot \frac{\partial^{2} A}{\partial y^{2}}(x, 0)+\cdots+ \\
& +\frac{y^{2 k-1}}{(2 k-1) !} \cdot \frac{\partial^{2 k-1} A}{\partial y^{2 k-1}}(x, 0)+\frac{y^{2 k}}{(2 k-1) !} \int_{0}^{1}(1-t)^{2 k-1} \cdot \frac{\partial^{2 k} A}{\partial y^{2 k}}(x, t y) d t \\
= & A_{0}(x)+y \cdot A_{1}(x)+\frac{1}{2 !} \cdot y^{2} A_{2}(x)+\cdots+\frac{y^{2 k-1}}{(2 k-1) !} \cdot A_{2 k-1}(x)+y^{2 k} \cdot A_{2 k}(x, y),
\end{aligned}
$$

sendo $A_{0}=A(x, 0), A_{1}(x)=\frac{\partial A}{\partial y}(x, 0), \cdots, A_{2 k-1}=\frac{\partial^{2 k-1} A}{\partial y^{2 k-1}}(x, 0)$ e

$A_{2 k}(x, y)=\frac{1}{(2 k-1) !} \int_{0}^{1}(1-t)^{2 k-1} \cdot \frac{\partial^{2 k} A}{\partial y^{2 k}}(x, t y) d t$.

Note que $A_{0} \in C^{2 k+\sigma}(\mathbb{R}), A_{1} \in C^{2 k-1+\sigma}(\mathbb{R}), \cdots, A_{2 k} \in C^{\sigma}\left(\mathbb{R}^{2}\right)$, com $0<\sigma<1$.

Analogamente, temos

$$
\begin{aligned}
B(x, y)= & B(x, 0)+y \cdot \frac{\partial B}{\partial y}(x, 0)+\frac{1}{2 !} \cdot y^{2} \cdot \frac{\partial^{2} B}{\partial y^{2}}(x, 0)+\cdots+ \\
& +\frac{y^{2 k-1}}{(2 k-1) !} \cdot \frac{\partial^{2 k-1} B}{\partial y^{2 k-1}}(x, 0)+\frac{y^{2 k}}{(2 k-1) !} \int_{0}^{1}(1-t)^{2 k-1} \cdot \frac{\partial^{2 k} B}{\partial y^{2 k}}(x, t y) d t \\
= & 0+y \cdot 0+\frac{1}{2 !} \cdot y^{2} \cdot 0+\cdots+\frac{y^{2 k-1}}{(2 k-1) !} \cdot 0+\frac{y^{2 k}}{(2 k-1) !} \int_{0}^{1}(1-t)^{2 k-1} \cdot \frac{\partial^{2 k} B}{\partial y^{2 k}}(x, t y) d t \\
= & y^{2 k} \cdot B_{2 k}(x, y),
\end{aligned}
$$

sendo $B_{2 k}(x, y)=\frac{1}{(2 k-1) !} \int_{0}^{1}(1-t)^{2 k-1} \cdot \frac{\partial^{2 k} B}{\partial y^{2 k}}(x, t y) d t ;$ daí, $B_{2 k} \in C^{\sigma}\left(\mathbb{R}^{2}\right)$, com $0<\sigma<1$. 
Assim, temos

$$
\begin{aligned}
L_{k} \omega & =\left[A_{0}(x)+y A_{1}(x)+\frac{1}{2 !} y^{2} A_{2}(x) \cdots+y^{2 k} A_{2 k}(x, y)\right] \cdot \omega+y^{2 k} B_{2 k}(x, y) \cdot \bar{\omega} \\
& =y^{2 k} \cdot A_{2 k}(x, y) \cdot \omega+y^{2 k} \cdot B_{2 k}(x, y) \cdot \bar{\omega}+\left[A_{0}(x)+\cdots+\frac{y^{2 k-1}}{(2 k-1) !} A_{2 k-1}(x)\right] \cdot \omega \cdot(* 1)
\end{aligned}
$$

Agora, vamos transformar $(* 1)$ numa equação da forma

$$
V_{k} \mu=y^{2 k} \cdot P(x, y) \cdot \mu+y^{2 k} \cdot Q(x, y) \cdot \bar{\mu},
$$

sendo $P, Q \in C^{\sigma}$ numa vizinhança de $(0,0)$.

Para isso, considere uma vizinhança $U_{\epsilon, \delta}=(-\epsilon, \epsilon) \times(-\delta, \delta) \subset \mathbb{R}^{2}$ e vamos procurar $\omega$ na forma $\omega=\mu \cdot \nu$, com $\mu \in C^{1}\left(U_{\epsilon, \delta}\right) ; \nu \in C^{1+\sigma}\left(U_{\epsilon, \delta}\right)$ e $\nu \neq 0$. Assim, temos em (*1) o seguinte:

$L_{k}(\mu \cdot \nu)=y^{2 k} A_{2 k}(x, y) \cdot \mu \cdot \nu+y^{2 k} B_{2 k}(x, y) \cdot \overline{\mu \cdot \nu}+\left[A_{0}(x)+\cdots+\frac{y^{2 k-1}}{(2 k-1) !} A_{2 k-1}(x)\right] \cdot \mu \cdot \nu$

para todo $(x, y) \in U_{\epsilon, \delta}$.

Logo, pela regra de Leibniz, temos

$$
\begin{aligned}
\left(L_{k} \mu\right) \cdot \nu+\mu \cdot\left(L_{k} \nu\right)= & y^{2 k} \cdot A_{2 k}(x, y) \cdot \mu \cdot \nu+y^{2 k} \cdot B_{2 k}(x, y) \cdot \bar{\mu} \cdot \bar{\nu}+ \\
& +\left[A_{0}(x)+\cdots+\frac{y^{2 k-1}}{(2 k-1) !} A_{2 k-1}(x)\right] \cdot \mu \cdot \nu
\end{aligned}
$$

para todo $(x, y) \in U_{\epsilon, \delta}$.

Daí,

$$
\begin{aligned}
L_{k} \mu= & -\frac{\mu}{\nu} \cdot L_{k} \nu+y^{2 k} \cdot A_{2 k}(x, y) \cdot \mu+y^{2 k} \cdot B_{2 k}(x, y) \cdot \bar{\mu} \cdot \frac{\bar{\nu}}{\nu}+ \\
& +\left[A_{0}(x)+\cdots+\frac{1}{(2 k-1) !} \cdot y^{2 k-1} \cdot A_{2 k-1}(x)\right] \cdot \mu \\
= & y^{2 k} \cdot A_{2 k}(x, y) \cdot \mu+y^{2 k} \cdot \tilde{B}_{2 k}(x, y) \cdot \bar{\mu}+ \\
& +\left[A_{0}(x)+\cdots+\frac{1}{(2 k-1) !} \cdot y^{2 k-1} \cdot A_{2 k-1}(x)-\frac{1}{\nu} \cdot L_{k} \nu\right] \cdot \mu
\end{aligned}
$$


sendo $\tilde{B}_{2 k}=B_{2 k} \cdot \frac{\bar{\nu}}{\nu} \in C^{\sigma}\left(U_{\epsilon, \delta}\right)$.

Assim,

$L_{k} \mu=y^{2 k} A_{2 k}(x, y) \cdot \mu+y^{2 k} \tilde{B}_{2 k}(x, y) \cdot \bar{\mu}+\left[\frac{\left(A_{0}(x)+\cdots+\frac{y^{2 k-1}}{(2 k-1) !} A_{2 k-1}(x)\right) \nu-L_{k} \nu}{\nu}\right] \cdot \mu,(* 2)$

para todo $(x, y) \in U_{\epsilon, \delta}$.

Logo, basta encontrar $\nu$, com $\nu \neq 0$, que satisfaça

$$
L_{k} \nu(x, y)-\left(A_{0}(x)+y A_{1}(x) \cdots+\frac{y^{2 k-1}}{(2 k-1) !} A_{2 k-1}(x)\right) \cdot \nu(x, y)=y^{2 k} \cdot g(x, y),
$$

para todo $(x, y) \in U_{\epsilon, \delta}$ e para alguma $g \in C^{\sigma}\left(U_{\epsilon, \delta}\right)$.

Para isso, vamos procurar $\nu$ na forma

$$
\nu(x, y)=1+\alpha_{1}(x) \cdot y+\cdots+\alpha_{2 k}(x) \cdot y^{2 k}=1+\sum_{m=1}^{2 k} \alpha_{m}(x) \cdot y^{m},
$$

sendo $\alpha_{1} \in C^{2 k+\alpha}((-\epsilon, \epsilon)), \cdots, \alpha_{2 k} \in C^{1+\alpha}((-\epsilon, \epsilon))$, para todo $(x, y) \in U_{\epsilon, \delta}$.

Observe que para $\nu$ da forma $(* 4)$ tem-se $\nu(0,0)=1$; logo, para $\epsilon, \delta>0$ suficientemente pequenos, temos $\nu(x, y)>0, \forall(x, y) \in U_{\epsilon, \delta}$.

Note que:

$$
\begin{aligned}
&-L_{k} \nu(x, y)=\sum_{m=1}^{2 k} m \cdot \alpha_{m}(x) \cdot y^{m-1}-i(2 k+1) \sum_{m=1}^{2 k} \alpha_{m}^{\prime}(x) \cdot y^{m+2 k} \\
& \text { - }\left[A_{0}(x)+\cdots+\frac{y^{2 k-1} A_{2 k-1}(x)}{(2 k-1) !}\right] \cdot \nu=\left[\sum_{j=0}^{2 k-1} \frac{1}{j !} \cdot y^{j} A_{j}(x)\right]\left[1+\sum_{m=1}^{2 k} \alpha_{m}(x) \cdot y^{m}\right] \\
&=\sum_{j=0}^{2 k-1} \frac{y^{j}}{j !} A_{j}(x)+\sum_{j=0}^{2 k-1} \sum_{m=1}^{2 k} \frac{y^{j+m}}{j !} A_{j}(x) \alpha_{m}(x),
\end{aligned}
$$

para todo $(x, y) \in U_{\epsilon, \delta}$. 
Logo, temos

$$
\begin{aligned}
& L_{k} \nu-\left[A_{0}(x)+\cdots+\frac{\left.y^{2 k-1} A_{2 k-1}(x)\right] \nu}{(2 k-1) !}=\left[\alpha_{1}(x)-A_{0}(x)\right]+\right. \\
&+\left.y\left[2 \alpha_{2}(x)-A_{0}(x) \alpha_{1}(x)-A_{1}(x)\right)\right]+ \\
&+y^{2}\left[3 \alpha_{3}(x)-A_{0}(x) \alpha_{2}(x)-A_{1}(x) \alpha_{1}(x)+\frac{A_{2}(x)}{2 !}\right]+ \\
& \vdots \\
&+y^{2 k-1}\left[2 k \alpha_{2 k}(x)-A_{0}(x) \alpha_{2 k-1}(x)-\cdots-\frac{A_{2 k-1}(x)}{(2 k-1) !}+\right. \\
&-y^{2 k}\left\{\alpha_{2 k}(x) A_{0}(x)+\cdots+\frac{A_{2 k-1}(x) \alpha_{1}(x)}{(2 k-1) !}+\right. \\
&+y^{2}\left[\alpha_{2 k}(x) A_{1}(x)+\cdots+\frac{A_{2 k-1}(x) \alpha_{2}(x)}{(2 k-1) !}\right]+ \\
&+y^{2}\left[\frac{1}{2 !} \alpha_{2 k}(x) A_{2}(x)+\cdots+\frac{A_{2 k-1}(x) \alpha_{3}(x)}{(2 k-1) !}\right]+ \\
& \vdots \\
&+y^{2 k-1}\left[\frac{1}{(2 k-1) !} A_{2 k-1}(x) \alpha_{2 k}(x)\right]+ \\
&\left.+\left(\alpha_{1}^{\prime}(x) y+\cdots+\alpha_{2 k}^{\prime}(x) y^{2 k}\right) \cdot(2 k+1) i\right\} \\
&
\end{aligned}
$$

para todo $(x, y) \in U_{\epsilon, \delta}$. Consequentemente, de $(* 3)$ temos o seguinte sistema de equações

$$
\begin{cases}\alpha_{1}(x)-A_{0}(x) & =0 \\ 2 \alpha_{2}(x)-A_{0}(x) \alpha_{1}(x)-A_{1}(x) & =0 \\ 3 \alpha_{3}(x)-A_{0}(x) \alpha_{2}(x)-A_{1}(x) \alpha_{1}(x)+\frac{A_{2}(x)}{2 !} & =0 \\ 2 k \alpha_{2 k}(x)-A_{0}(x) \alpha_{2 k-1}(x)-\cdots-\frac{1}{(2 k-1) !} A_{2 k-1}(x) & \vdots \\ -\left[\alpha_{2 k}(x) A_{0}(x)+\cdots+\frac{1}{(2 k-1) !} A_{2 k-1}(x) \alpha_{1}(x)\right]+ & \\ -y\left[\alpha_{2 k}(x) A_{1}(x)+\cdots+\frac{A_{2 k-1}(x) \alpha_{2 k}(x)}{(2 k-1) !}\right]-\cdots-\frac{y^{2 k-1} A_{2 k-1}(x) \alpha_{2 k}(x)}{(2 k-1) !}+ & \end{cases}
$$


para todo $(x, y) \in U_{\epsilon, \delta}$.

Daí,

$$
\begin{aligned}
& \alpha_{1}(x)=A_{0}(x) \in C^{2 k+\sigma}((-\epsilon, \epsilon)) \\
& \alpha_{2}(x)=\frac{1}{2 !}\left[A_{0}^{2}(x)+A_{1}(x)\right] \in C^{2 k-1+\sigma}((-\epsilon, \epsilon)) \\
& \alpha_{3}(x)= \frac{1}{3 !}\left[A_{0}^{3}(x)+3 A_{0}(x) A_{1}(x)+A_{2}(x)\right] \in C^{2 k-2+\sigma}((-\epsilon, \epsilon)), \\
& \alpha_{4}(x)=\frac{1}{4 !}\left[A_{0}^{4}(x)+6 A_{0}^{2}(x) A_{1}(x)+4 A_{0}(x) A_{2}(x)+3 A_{1}^{2}(x)+A_{3}(x)\right] \in C^{2 k-3+\sigma}((-\epsilon, \epsilon)), \\
& \vdots \\
& \alpha_{2 k}(x)=\frac{1}{(2 k) !}\left[A_{0}^{2 k}(x)+\cdots+A_{2 k-1}(x)\right] \in C^{1+\sigma}((-\epsilon, \epsilon)),
\end{aligned}
$$

Portanto,

$\nu(x, y)=1+A_{0}(x) \cdot y+\frac{1}{2 !}\left[A_{0}^{2}(x)+A_{1}(x)\right] y^{2}+\cdots+\frac{1}{(2 k) !}\left[A_{0}^{2 k}(x)+\cdots+A_{2 k-1}(x)\right] y^{2 k}$,

para todo $(x, y) \in U_{\epsilon, \delta}$. Logo, $\nu \in C^{1+\sigma}\left(U_{\epsilon, \delta}\right)$ e satisfaz as propriedades requeridas.

Também, substituindo as expressões encontradas para $\alpha_{i}$, com $i=0, \cdots, 2 k$ na última equação do sistema $(* 5)$, obtemos $g \in C^{\sigma}\left(U_{\epsilon, \delta}\right)$.

Finalmente, usando $\nu$ obtida acima em $(* 2)$ temos

$$
\begin{aligned}
L_{k} \mu & =y^{2 k} \cdot A_{2 k}(x, y) \cdot \mu+y^{2 k} \cdot \tilde{B}_{2 k}(x, y) \cdot \bar{\mu}-y^{2 k} \cdot \frac{1}{\nu} \cdot g(x, y) \cdot \mu \\
& =y^{2 k}\left[A_{2 k}(x, y)-\frac{1}{\nu} \cdot g(x, y)\right] \cdot \mu+y^{2 k} \cdot \tilde{B}_{2 k}(x, y) \cdot \bar{\mu} \\
& =y^{2 k}\left[\frac{A_{2 k}(x, y) \cdot \nu-g(x, y)}{\nu}\right] \cdot \mu+y^{2 k} \cdot \tilde{B}_{2 k}(x, y) \cdot \bar{\mu}
\end{aligned}
$$

Considerando $P(x, y)=\frac{A_{2 k}(x, y) \cdot \nu(x, y)-g(x, y)}{\nu(x, y)} \quad$ e $\quad Q(x, y)=\tilde{B}_{2 k}(x, y)$ temos a equação

$$
L_{k} \mu=y^{2 k} \cdot P(x, y) \cdot \mu+y^{2 k} \cdot Q(x, y) \cdot \bar{\mu},
$$


sendo $P, Q \in C^{\sigma}\left(U_{\epsilon, \delta}\right)$.

Portanto, encontrar $\omega \in C^{1}\left(U_{\epsilon, \delta}\right)$ tal que $\omega$ seja solução da equação (3.8) é equivalente a encontrar $\mu \in C^{1}\left(U_{\epsilon, \delta}\right)$ tal que $\mu$ satisfaça a equação $(* 6)$.

Considere a função

$$
\begin{aligned}
z_{k}: \quad U_{\epsilon, \delta} & \rightarrow z_{k}\left(U_{\epsilon, \delta}\right) \subset \mathbb{C} \\
(x, y) & \mapsto z_{k}(x, y)=x+i y^{2 k+1} .
\end{aligned}
$$

Notemos que $z_{k}\left(U_{\epsilon, \delta}\right)=\left\{x+i y^{2 k+1}:|x| \leq \epsilon,|y| \leq \delta\right\}$ e que $0 \in z_{k}\left(U_{\epsilon, \delta}\right)$.

Por outro lado, para todo $(x, y) \in U_{\epsilon, \delta}$, temos que:

$$
\begin{aligned}
& -L_{k}\left(\bar{z}_{k}(x, y)\right)=\frac{\partial}{\partial y}\left(x-i y^{2 k+1}\right)-i(2 k+1) y^{2 k} \frac{\partial}{\partial x}\left(x-i y^{2 k+1}\right)=-2 i(2 k+1) y^{2 k} \\
& \text { - } L_{k}\left(z_{k}(x, y)\right)=\frac{\partial}{\partial y}\left(x+i y^{2 k+1}\right)-i(2 k+1) y^{2 k} \frac{\partial}{\partial x}\left(x+i y^{2 k+1}\right)=0 .
\end{aligned}
$$

Logo, o push-forward de $L_{k} \mu$ pela função $z_{k}$ é definido por $z_{k *}\left(L_{k} \mu\right)=\left(L_{k} \mu\right) \circ z_{k}^{-1}$, para qualquer $L_{k} \mu \in C^{\infty}\left(U_{\epsilon, \delta}\right)$.

Assim, em $(* 6)$ temos

$$
\begin{aligned}
\left(L_{k} \mu\right) \circ z_{k}^{-1} & =\left(y^{2 k} \cdot P \cdot \mu\right) \circ z_{k}^{-1}+\left(y^{2 k} \cdot Q \cdot \bar{\mu}\right) \circ z_{k}^{-1} \\
& =\left[\left(y^{2 k} P\right) \circ z_{k}^{-1}\right] \cdot\left[\mu \circ z_{k}^{-1}\right]+\left[\left(y^{2 k} Q\right) \circ z_{k}^{-1}\right] \cdot\left[\bar{\mu} \circ z_{k}^{-1}\right] .
\end{aligned}
$$

Agora, considerando os pontos de $z_{k}\left(U_{\epsilon, \delta}\right)$ escritos na forma $z_{k}=\xi+i \eta$ temos

$$
\begin{gathered}
\xi=\frac{z_{k}+\bar{z}_{k}}{2} \quad \text { e } \eta=\frac{z_{k}-\bar{z}_{k}}{2 i} \\
\frac{\partial}{\partial \xi}=\frac{\partial}{\partial z_{k}}+\frac{\partial}{\partial \bar{z}_{k}} \quad \text { e } \quad \frac{\partial}{\partial \eta}=i\left(\frac{\partial}{\partial z_{k}}-\frac{\partial}{\partial \bar{z}_{k}}\right) .
\end{gathered}
$$

Considere $\tilde{L}_{k}: C^{\infty}\left(z_{k}\left(U_{\epsilon, \delta}\right)\right) \rightarrow C^{\infty}\left(z_{k}\left(U_{\epsilon, \delta}\right)\right)$ o campo vetorial da mudança de coordenadas 
definido por

$$
\begin{aligned}
\tilde{L}_{k} & =L_{k} \xi\left(z_{k}^{-1}(\xi, \eta)\right) \cdot \frac{\partial}{\partial \xi}+L_{k} \eta\left(z_{k}^{-1}(\xi, \eta)\right) \cdot \frac{\partial}{\partial \eta} \\
& =L_{k} \xi\left(z_{k}^{-1}\left(x, y^{2 k+1}\right)\right) \cdot \frac{\partial}{\partial \xi}+L_{k} \eta\left(z_{k}^{-1}\left(x, y^{2 k+1}\right)\right) \cdot \frac{\partial}{\partial \eta} \\
& =L_{k} \xi(x, y) \cdot \frac{\partial}{\partial \xi}+L_{k} \eta(x, y) \cdot \frac{\partial}{\partial \eta} \\
& =\left(L_{k}\left(\frac{z_{k}+\bar{z}_{k}}{2}\right)\right)(x, y) \cdot\left[\frac{\partial}{\partial z_{k}}+\frac{\partial}{\partial \bar{z}_{k}}\right]+\left(L_{k}\left(\frac{z_{k}-\bar{z}_{k}}{2 i}\right)\right)(x, y) \cdot\left[\frac{\partial}{\partial z_{k}}-\frac{\partial}{\partial \bar{z}_{k}}\right] i \\
& =\frac{1}{2}\left(L_{k} z_{k}+L_{k} \bar{z}_{k}\right)(x, y) \cdot\left[\frac{\partial}{\partial z_{k}}+\frac{\partial}{\partial \bar{z}_{k}}\right]+\frac{1}{2}\left(L_{k} z_{k}-L_{k} \bar{z}_{k}\right)(x, y) \cdot\left[\frac{\partial}{\partial z_{k}}-\frac{\partial}{\partial \bar{z}_{k}}\right] \\
& =L_{k} z_{k}(x, y) \cdot \frac{\partial}{\partial z_{k}}+L_{k} \bar{z}_{k}(x, y) \cdot \frac{\partial}{\partial \bar{z}_{k}},
\end{aligned}
$$

para todo $(x, y) \in U_{\epsilon, \delta}$.

Logo,

$$
\tilde{L}_{k}\left(\mu \circ z_{k}^{-1}\right)=L_{k} z_{k}(x, y) \cdot \frac{\partial}{\partial z_{k}}\left(\mu \circ z_{k}^{-1}\right)+L_{k} \bar{z}_{k}(x, y) \cdot \frac{\partial}{\partial \bar{z}_{k}}\left(\mu \circ z_{k}^{-1}\right)
$$

para todo $(x, y) \in U_{\epsilon, \delta}$.

Por outro lado, pela proposição 1.3 temos que $\tilde{L}_{k}\left(\mu \circ z_{k}^{-1}\right)=\left(L_{k} \mu\right) \circ z_{k}^{-1}$.

Assim, de $(* 10)$ e $(* 9)$ temos

$$
\begin{aligned}
L_{k} z_{k}(x, y) \frac{\partial}{\partial z_{k}}\left(\mu \circ z_{k}^{-1}\right)+L_{k} \bar{z}_{k}(x, y) \frac{\partial}{\partial \bar{z}_{k}}\left(\mu \circ z_{k}^{-1}\right)= & {\left[\left(y^{2 k} P\right) \circ z_{k}^{-1}\right] \cdot\left[\mu \circ z_{k}^{-1}\right]+} \\
& +\left[\left(y^{2 k} Q\right) \circ z_{k}^{-1}\right] \cdot\left[\bar{\mu} \circ z_{k}^{-1}\right],
\end{aligned}
$$

para todo $(x, y) \in U_{\epsilon, \delta}$.

Considere $\psi=\mu \circ z_{k}^{-1}: z_{k}\left(U_{\epsilon, \delta}\right) \rightarrow \mathbb{C}$ e $\bar{\psi}=\bar{\mu} \circ z_{k}^{-1}: z_{k}\left(U_{\epsilon, \delta}\right) \rightarrow \mathbb{C}$. Como $z_{k} \in C^{\infty}\left(U_{\epsilon, \delta}\right)$ e $\mu \in C^{1}\left(U_{\epsilon, \delta}\right)$ temos que $\psi \in C^{1}\left(z_{k}\left(U_{\epsilon, \delta}\right)\right)$. Logo, substituindo em $(* 11)$ temos

$$
\underbrace{L_{k} z_{k}(x, y)}_{=0} \cdot \frac{\partial \psi}{\partial z_{k}}+L_{k} \bar{z}_{k}(x, y) \cdot \frac{\partial \psi}{\partial \bar{z}_{k}}=y^{2 k} \cdot P\left(z_{k}^{-1}(\xi, \eta)\right) \cdot \psi+y^{2 k} \cdot Q\left(z_{k}^{-1}(\xi, \eta)\right) \cdot \bar{\psi}
$$


o que implica

$$
-2 i(2 k+1) y^{2 k} \cdot \frac{\partial \psi}{\partial \bar{z}_{k}}=y^{2 k} \cdot P(\xi, \sqrt[2 k+1]{\eta}) \cdot \psi+y^{2 k} \cdot Q(\xi, \sqrt[2 k+1]{\eta}) \cdot \bar{\psi}
$$

daí,

$$
\frac{\partial \psi}{\partial \bar{z}_{k}}=\frac{i}{2(2 k+1)}[P(\xi, \sqrt[2 k+1]{\eta}) \cdot \psi+Q(\xi, \sqrt[2 k+1]{\eta}) \cdot \bar{\psi}]
$$

Sabemos que $\psi \in C^{1}\left(z_{k}\left(U_{\epsilon, \delta}\right)\right)$ e $P \circ z_{k}^{-1}, Q \circ z_{k}^{-1} \in C^{\sigma}\left(z_{k}\left(U_{\epsilon, \delta}\right)\right)$.

Agora, considere a função $\phi: \mathbb{C} \rightarrow \mathbb{C}$ tais que:

- $\phi \in C_{c}^{\infty}(\mathbb{C})$;

- $\operatorname{Supp}(\phi) \subset z_{k}\left(U_{\epsilon, \delta}\right)$;

- $\phi \equiv 1$ num domínio compacto $\bar{V}$ de $(0,0), \operatorname{com} \bar{V} \subset z_{k}\left(U_{\epsilon, \delta}\right)$.

Considere em $z_{k}\left(U_{\epsilon, \delta}\right)$ a seguinte equação

$$
\frac{\partial \vartheta}{\partial \bar{z}_{k}}=\frac{i}{6}[\tilde{P}(\xi, \eta) \cdot \vartheta+\tilde{Q}(\xi, \eta) \cdot \bar{\vartheta}]
$$

sendo $\tilde{P}, \tilde{Q}: \mathbb{C} \rightarrow \mathbb{C}$ dados por:

$$
\begin{aligned}
& \tilde{P}(\xi, \eta)=\left\{\begin{array}{cl}
\left(P \circ z_{k}^{-1}\right)(\xi, \eta) \cdot \phi(\xi, \eta) & , \quad(\xi, \eta) \in z_{k}\left(U_{\epsilon, \delta}\right) \\
0 & , \quad(\xi, \eta) \in \mathbb{C} \backslash z_{k}\left(U_{\epsilon, \delta}\right)
\end{array},\right. \\
& \tilde{Q}(\xi, \eta)=\left\{\begin{array}{cl}
\left(Q \circ z_{k}^{-1}\right)(\xi, \eta) \cdot \phi(\xi, \eta) & , \quad(\xi, \eta) \in z_{k}\left(U_{\epsilon, \delta}\right) \\
0 & , \quad(\xi, \eta) \in \mathbb{C} \backslash z_{k}\left(U_{\epsilon, \delta}\right)
\end{array} .\right.
\end{aligned}
$$

Note que $\tilde{P}, \tilde{Q} \in C^{\sigma}(\mathbb{C})$.

Assim, como $\psi$ satisfaz $(* 13)$, para todo $(\xi, \eta) \in V$, então $\psi$ é uma $[\tilde{P}, \tilde{Q}]$-função pseudoanalítica em $V$. Logo, pelo teorema 2.3 (Princípio da similaridade clássico), temos que existe uma função analítica $h$ no interior de $V$ e $s_{0} \in C^{\sigma}(\bar{V})$ tal que

$$
\psi(\xi, \eta)=e^{s_{0}(\xi, \eta)} \cdot h(\xi, \eta)
$$

para todo $(\xi, \eta) \in V$. 
Finalmente, compondo novamente temos $\left(\psi \circ z_{k}\right)=e^{s_{0} \circ z_{k}} \cdot\left(h \circ z_{k}\right)$.

Assim, dado $(x, y) \in U=z_{k}^{-1}(V)$, temos

$$
\left(\psi \circ z_{k}\right)(x, y)=e^{\left(s_{0} \circ z_{k}\right)(x, y)} \cdot\left(h \circ z_{k}\right)(x, y)
$$

isto é,

$$
\mu(x, y)=e^{\left(s_{0} \circ z_{k}\right)(x, y)} \cdot h\left(z_{k}(x, y)\right)
$$

Logo, temos que $(* 15)$ é solução da equação $(* 6)$. Mas, sabemos que $\mu=\frac{\omega}{\nu}, \operatorname{com} \nu \neq 0$.

Assim, em (*15), temos

$$
\frac{\omega(x, y)}{\nu(x, y)}=e^{\left(s_{0} \circ z_{k}\right)(x, y)} \cdot h\left(x+i y^{2 k+1}\right)
$$

o que implica

$$
\omega(x, y)=\nu(x, y) \cdot e^{\left(s_{0} \circ z_{k}\right)(x, y)} \cdot h\left(x+i y^{2 k+1}\right)
$$

daí,

$$
\omega(x, y)=e^{\left(s_{0} \circ z_{k}\right)(x, y)+\log \nu(x, y)} \cdot h\left(x+i y^{2 k+1}\right),
$$

para todo $(x, y) \in U=z_{k}^{-1}(V)$.

Portanto, de $(* 16)$ temos que $\omega$ tem a forma desejada.

Reciprocamente, com a mesma notação que acima, seja $h$ uma função holomorfa em $V \subset z_{k}\left(U_{\epsilon, \delta}\right)$. Logo, pelo teorema 2.4 (Princípio da similaridade clássico), temos que existe uma função $s_{0} \in C^{\sigma}(\bar{V})$ tal que $\psi(\xi, \eta)=e^{s_{0}(\xi, \eta)} \cdot h(\xi, \eta)$ é $[\tilde{P}, \tilde{Q}]$-pseudo-analítica em $V$, isto é, $\psi \in C^{1}(V)$ e satisfaz a equação $(* 13)$. Logo, como $\psi=\mu \circ z_{k}^{-1}$, segue que a função dada por $(* 16)$ satisfaz (3.8) para todo $(x, y) \in U=z_{k}^{-1}(V)$. 


\subsection{Princípio da similaridade para um campo vetorial complexo de tipo infinito}

Seja

$$
L=\frac{\partial}{\partial y}-i x \frac{\partial}{\partial x}
$$

um campo vetorial complexo definido em $\mathbb{R}^{2}$.

Note que $\operatorname{char}(L)=\{(0, y): y \in \mathbb{R}\} ;$ de fato, $1 \cdot x=0 \Leftrightarrow x=0$. Portanto, $L$ é não elítico. Afirmamos que $L$ é de tipo infinito em $(0, y) \in \operatorname{char}(L)$.

De fato, temos

$$
\bar{L}=\frac{\partial}{\partial y}+i x \frac{\partial}{\partial x}
$$

Assim,

$$
\begin{aligned}
C^{1} & =[L, \bar{L}]=L \cdot \bar{L}-\bar{L} \cdot L \\
& =\left(\frac{\partial}{\partial y}-i x \frac{\partial}{\partial x}\right) \cdot\left(\frac{\partial}{\partial y}+i x \frac{\partial}{\partial x}\right)-\left(\frac{\partial}{\partial y}+i x \frac{\partial}{\partial x}\right) \cdot\left(\frac{\partial}{\partial y}-i x \frac{\partial}{\partial x}\right)=0 .
\end{aligned}
$$

Logo, $C^{m}=0, \forall m \geq 1$.

Portanto, $L$ é de tipo infinito em $(0, y) \in \operatorname{char}(L)$.

Teorema 3.3. Sejam

$$
L=\frac{\partial}{\partial y}-i x \frac{\partial}{\partial x}, \quad z(x, y)=x e^{i y}
$$

e $A, B \in C^{2+\sigma}\left(\mathbb{R}^{2}\right)$, com $0<\sigma<1$, tais que

$$
B(0, y)=\frac{\partial B}{\partial x}(0, y)=0, \forall y \in \mathbb{R}
$$

Então, existe um conjunto aberto, conexo e limitado $U \subset \mathbb{R}^{2}$ contendo a origem tal que qualquer $\omega \in C^{1}(\bar{U})$ solução da equação

$$
L \omega=A \cdot \omega+B \cdot \bar{\omega}
$$

tem a forma

$$
\omega(x, y)=e^{s(x, y)} \cdot H(z(x, y))
$$

para alguma função holomorfa $H$ definida no interior de $z(U), H \in C^{\sigma}(z(\bar{U}))$ e alguma função $s \in C^{\sigma}(\bar{U})$. 
Reciprocamente, para cada função holomorfa $H$ definida no interior de $z(U)$ e $H \in C^{\sigma}(z(\bar{U}))$, existe $s \in C^{\sigma}(\bar{U})$ tal que a função $\omega(x, y)=e^{s(x, y)} \cdot H(z(x, y))$ satisfaz a equação (3.13).

\section{Demonstração.}

Sejam $A, B \in C^{2+\sigma}\left(\mathbb{R}^{2}\right)$, com $0<\sigma<1$. Fixemos $p=(0, y) \in \mathbb{R}^{2}$; logo, pela fórmula de Taylor com resto integral temos

$$
\begin{aligned}
A(x, y)= & A(0, y)+\left(\frac{\partial A}{\partial x}\right)_{p}(x-0)+\left(\frac{\partial A}{\partial y}\right)_{p}(y-y)+\int_{0}^{1}(1-t)\left[\left(\frac{\partial^{2} A}{\partial y^{2}}\right)_{p}(y-y)^{2}+\right. \\
& \left.+\left(\frac{\partial^{2} A}{\partial y \partial x}\right)_{p}(x-0)(y-y)+\frac{\partial^{2} A}{\partial x^{2}}(t x, y) \cdot(x-0)^{2}\right] d t \\
= & A(0, y)+x \cdot \frac{\partial A}{\partial x}(0, y)+x^{2} \int_{0}^{1}(1-t) \cdot \frac{\partial^{2} A}{\partial x^{2}}(t x, y) d t \\
= & A_{0}(y)+x A_{1}(y)+x^{2} A_{2}(x, y)
\end{aligned}
$$

sendo $A_{0}(x)=A(0, y), A_{1}(x)=\frac{\partial A}{\partial x}(0, y), A_{2}(x, y)=\int_{0}^{1}(1-t) \cdot \frac{\partial^{2} A}{\partial x^{2}}(t x, y) d t$.

Note que $A_{0} \in C^{2+\sigma}(\mathbb{R}), A_{1} \in C^{1+\sigma}(\mathbb{R})$ e $A_{2} \in C^{\sigma}\left(\mathbb{R}^{2}\right)$, com $0<\sigma<1$.

Analogamente, temos

$$
\begin{aligned}
B(x, y)= & B(0, y)+\left(\frac{\partial B}{\partial x}\right)_{p}(x-0)+\left(\frac{\partial B}{\partial y}\right)_{p}(y-y)+\int_{0}^{1}(1-t)\left[\left(\frac{\partial^{2} B}{\partial y^{2}}\right)_{p}(y-y)^{2}+\right. \\
& \left.+\left(\frac{\partial^{2} B}{\partial y \partial x}\right)_{p}(x-0)(y-y)+\frac{\partial^{2} B}{\partial x^{2}}(t x, y) \cdot(x-0)^{2}\right] d t \\
= & B(0, y)+x \cdot \frac{\partial B}{\partial x}(0, y)+x^{2} \int_{0}^{1}(1-t) \cdot \frac{\partial^{2} B}{\partial x^{2}}(t x, y) d t \\
= & 0+x \cdot 0+x^{2} B_{2}(x, y) \\
= & x^{2} \cdot B_{2}(x, y),
\end{aligned}
$$


sendo $B_{2}(x, y)=\int_{0}^{1}(1-t) \cdot \frac{\partial^{2} B}{\partial x^{2}}(t x, y) d t ;$ daí, $B_{2} \in C^{\sigma}\left(\mathbb{R}^{2}\right)$, com $0<\sigma<1$.

Assim, temos

$$
\begin{aligned}
L \omega & =\left[A_{0}(y)+x \cdot A_{1}(y)+x^{2} \cdot A_{2}(x, y)\right] \cdot \omega+x^{2} \cdot B_{2}(x, y) \cdot \bar{\omega} \\
& =x^{2} \cdot A_{2}(x, y) \cdot \omega+x^{2} \cdot B_{2}(x, y) \cdot \bar{\omega}+\left[A_{0}(y)+x \cdot A_{1}(y)\right] \cdot \omega .
\end{aligned}
$$

Agora, vamos transformar $(* 1)$ numa equação da forma

$$
L \mu=x^{2} \cdot P(x, y) \cdot \mu+x^{2} \cdot Q(x, y) \cdot \bar{\mu},
$$

sendo $P, Q \in C^{\sigma}$ numa vizinhança de $(0,0)$.

Para isso considere uma vizinhança aberta $U_{\epsilon, \delta}=(-\epsilon, \epsilon) \times(-\delta, \delta) \subset \mathbb{R}^{2}$ e vamos procurar $\omega$ na forma $\omega=\mu \cdot \nu, \operatorname{com} \mu \in C^{1}\left(U_{\epsilon, \delta}\right), \nu \in C^{1+\sigma}\left(U_{\epsilon, \delta}\right)$ e $\nu \neq 0$. Assim, temos em $(* 1)$ o seguinte:

$$
L(\mu \cdot \nu)=x^{2} \cdot A_{2}(x, y) \cdot \mu \cdot \nu+x^{2} \cdot B_{2}(x, y) \cdot \overline{\mu \cdot \nu}+\left[A_{0}(y)+x \cdot A_{1}(y)\right] \cdot \mu \cdot \nu,
$$

para todo $(x, y) \in U_{\epsilon, \delta}$.

Logo, pela regra de Leibniz, temos

$$
(L \mu) \cdot \nu+\mu \cdot(L \nu)=x^{2} \cdot A_{2}(x, y) \cdot \mu \cdot \nu+x^{2} \cdot B_{2}(x, y) \cdot \bar{\mu} \cdot \bar{\nu}+\left[A_{0}(y)+x \cdot A_{1}(y)\right] \cdot \mu \cdot \nu
$$

para todo $(x, y) \in U_{\epsilon, \delta}$.

Daí,

$$
\begin{aligned}
L \mu & =-\frac{\mu}{\nu} \cdot L \nu+x^{2} \cdot A_{2}(x, y) \cdot \mu+x^{2} \cdot B_{2}(x, y) \cdot \bar{\mu} \cdot \frac{\bar{\nu}}{\nu}+\left[A_{0}(y)+x \cdot A_{1}(y)\right] \cdot \mu \\
& =x^{2} \cdot A_{2}(x, y) \cdot \mu+x^{2} \cdot \tilde{B}_{2}(x, y) \cdot \bar{\mu}+\left[A_{0}(y)+x \cdot A_{1}(y)-\frac{1}{\nu} \cdot L \nu\right] \cdot \mu \\
& =x^{2} \cdot A_{2}(x, y) \cdot \mu+x^{2} \cdot \tilde{B}_{2}(x, y) \cdot \bar{\mu}+\left[\frac{\left(A_{0}(y)+x \cdot A_{1}(y)\right) \nu-L \nu}{\nu}\right] \cdot \mu,
\end{aligned}
$$

para todo $(x, y) \in U_{\epsilon, \delta}$, sendo $\tilde{B}_{2}=B_{2} \cdot \frac{\bar{\nu}}{\nu} \in C^{\sigma}\left(U_{\epsilon, \delta}\right)$. 
Logo, basta encontrar $\nu, \operatorname{com} \nu \neq 0$, que satisfaça

$$
L \nu(x, y)-\left[A_{0}(y)+x \cdot A_{1}(y)\right] \cdot \nu(x, y)=x^{2} \cdot g(x, y),
$$

para todo $(x, y) \in U_{\epsilon, \delta}$ e para alguma $g \in C^{\sigma}\left(U_{\epsilon, \delta}\right)$.

Para isso, vamos procurar $\nu$ na forma

$$
\nu(x, y)=\alpha_{0}(y)+x \cdot \alpha_{1}(y)
$$

para todo $(x, y) \in U_{\epsilon, \delta}$.

Note que:

- $L \nu(x, y)=\alpha_{0}{ }^{\prime}(y)+x \cdot \alpha_{1}^{\prime}(y)-i x \cdot \alpha_{1}(y) ;$

- $\left[A_{0}(y)+x A_{1}(y)\right] \cdot \nu(x, y)=A_{0}(y) \alpha_{0}(y)+A_{0}(y) \alpha_{1}(y) \cdot x+A_{1}(y) \alpha_{0}(y) \cdot x+$ $+A_{1}(y) \alpha_{1}(y) \cdot x^{2}$

para todo $(x, y) \in U_{\epsilon, \delta}$.

Logo, temos

$$
\begin{aligned}
L \nu(x, y)-\left[A_{0}(y)+x \cdot A_{1}(y)\right] \cdot \nu(x, y)= & {\left[\alpha_{0}{ }^{\prime}(y)-A_{0}(y) \alpha_{0}(y)\right]+\left[\alpha_{1}{ }^{\prime}(y)-i \alpha_{1}(y)+\right.} \\
& \left.-A_{0}(y) \alpha_{1}(y)-A_{1}(y) \alpha_{0}(y)\right] x+\left[-A_{1}(y) \alpha_{1}(y)\right] x^{2},
\end{aligned}
$$

para todo $(x, y) \in U_{\epsilon, \delta}$. Consequentemente, de $(* 3)$ temos o seguinte sistema de equações

$$
\begin{cases}\alpha_{0}{ }^{\prime}(y)-A_{0}(y) \cdot \alpha_{0}(y) & =0 \\ \alpha_{1}{ }^{\prime}(y)-i \alpha_{1}(y)-A_{0}(y) \cdot \alpha_{1}(y)-A_{1}(y) \cdot \alpha_{0}(y) & =0 \\ -A_{1}(y) \cdot \alpha_{1}(y) & =g(x, y)\end{cases}
$$

para todo $(x, y) \in U_{\epsilon, \delta}$.

Vamos utilizar o método de variação dos parâmetros em $(* 5)$; para isso, considere o fator integrante $\rho(y)=e^{-\int_{0}^{y} A_{0}(s) d s}$. Logo,

$$
\begin{aligned}
& \alpha_{0}{ }^{\prime}(y) \cdot \rho(y)-A_{0}(y) \cdot \alpha_{0}(y) \cdot \rho(y)=0 \cdot \rho(y) \quad \Rightarrow \\
& \frac{\partial}{\partial y}\left(\alpha_{0}(y) \cdot \rho(y)\right)=0 \quad \Rightarrow \\
& \int_{0}^{y} \frac{\partial}{\partial \tau}\left(\alpha_{0}(\tau) \cdot \rho(\tau)\right) d \tau=\int_{0}^{y} 0 \cdot d \tau=0
\end{aligned}
$$


Assim, pelo teorema fundamental do cálculo temos

$$
\begin{array}{rlrl}
\alpha_{0}(y) \cdot \rho(y)-\alpha_{0}(0) \cdot \rho(0) & =0 & \Rightarrow \\
\alpha_{0}(y) \cdot e^{-\int_{0}^{y} A_{0}(s) d s} & =\alpha_{0}(0) & \Rightarrow \\
\alpha_{0}(y) & =\alpha_{0}(0) \cdot e^{\int_{0}^{y} A_{0}(s) d s} . & & (* 8)
\end{array}
$$

Analogamente, usamos o método de variação dos parâmetros em $(* 6)$; para isso, considere o fator integrante $\rho(y)=e^{-\int_{0}^{y}\left(A_{0}(s)+i\right) d s}$. Logo,

$$
\begin{array}{rlrl}
\alpha_{1}{ }^{\prime}(y) \cdot \rho(y)-\alpha_{1}(y)\left[A_{0}(y)+i\right] \rho(y) & =A_{1}(y) \cdot \alpha_{0}(y) \cdot \rho(y) & \Rightarrow \\
\frac{\partial}{\partial y}\left(\alpha_{1}(y) \cdot \rho(y)\right) & =A_{1}(y) \cdot \alpha_{0}(y) \cdot \rho(y) & \Rightarrow \\
\int_{0}^{y} \frac{\partial}{\partial \tau}\left(\alpha_{1}(\tau) \cdot \rho(\tau)\right) d \tau & =\int_{0}^{y} A_{1}(\tau) \alpha_{0}(\tau) \cdot \rho(\tau) \cdot d \tau
\end{array}
$$

Assim, pelo teorema fundamental do cálculo temos

$$
\begin{aligned}
& \alpha_{1}(y) \cdot \rho(y)-\alpha_{1}(0) \cdot \rho(0)=\int_{0}^{y} A_{1}(\tau) \cdot \alpha_{0}(\tau) \cdot \rho(\tau) \cdot d \tau \\
& \alpha_{1}(y) \cdot e^{-\int_{0}^{y}\left[A_{0}(s)+i\right] d s}-\alpha_{1}(0)=\int_{0}^{y} A_{1}(\tau) \cdot \alpha_{0}(0) \cdot e^{\int_{0}^{\tau} A_{0}(s) d s} \cdot e^{-\int_{0}^{\tau}\left[A_{0}(s)+i\right] d s} d \tau \Rightarrow \\
& \alpha_{1}(y) \cdot e^{-\int_{0}^{y}\left[A_{0}(s)+i\right] d s}=\alpha_{1}(0)+\int_{0}^{y} A_{1}(\tau) \cdot \alpha_{0}(0) e^{-i \int_{0}^{\tau} d s} d \tau \quad \Rightarrow \\
& \alpha_{1}(y)=\left[\alpha_{1}(0)+\int_{0}^{y} A_{1}(\tau) \cdot \alpha_{0}(0) e^{-i \tau} d \tau\right] e^{\int_{0}^{y}\left[A_{0}(s)+i\right] d s} .
\end{aligned}
$$

Considere as seguintes condições iniciais:

$$
\alpha_{0}(0)=\alpha_{1}(0)=1
$$


Logo, em $(* 8),(* 9)$ e $(* 5)$ temos

$$
\begin{aligned}
& \alpha_{0}(y)=e^{\int_{0}^{y} A_{0}(s) d s} \in C^{2+\sigma}((-\delta, \delta)), \\
& \alpha_{1}(y)=\left[1+\int_{0}^{y} A_{1}(\tau) \cdot e^{-i \tau} d \tau\right] e^{\int_{0}^{y}\left[A_{0}(s)+i\right] d s} \in C^{1+\sigma}((-\delta, \delta)), \\
& g(x, y)=-A_{1}(y) \cdot\left[1+\int_{0}^{y} A_{1}(\tau) \cdot e^{-i \tau} d \tau\right] e^{\int_{0}^{y}\left[A_{0}(s)+i\right] d s} \in C^{1+\sigma}\left(U_{\epsilon, \delta}\right) .
\end{aligned}
$$

Portanto,

$$
\nu(x, y)=e^{\int_{0}^{y} A_{0}(s) d s}+x\left[1+\int_{0}^{y} A_{1}(\tau) \cdot e^{-i \tau} d \tau\right] e^{\int_{0}^{y}\left[A_{0}(s)+i\right] d s},
$$

para todo $(x, y) \in U_{\epsilon, \delta} ;$ também $\nu \in C^{1+\sigma}\left(U_{\epsilon, \delta}\right)$ e $\nu(0,0)=1$. Logo, para $\epsilon, \delta>0$ suficientemente pequenos temos $\nu(x, y)>0, \forall(x, y) \in U_{\epsilon, \delta}$.

Finalmente, usando $\nu$ obtida acima em $(* 2)$ temos

$$
\begin{aligned}
L \mu & =x^{2} \cdot A_{2}(x, y) \cdot \mu+x^{2} \cdot \tilde{B}_{2}(x, y) \cdot \bar{\mu}-x^{2} \cdot \frac{1}{\nu} \cdot g(x, y) \cdot \mu \\
& =x^{2}\left[A_{2}(x, y)-\frac{1}{\nu} \cdot g(x, y)\right] \cdot \mu+x^{2} \cdot \tilde{B}_{2}(x, y) \cdot \bar{\mu} \\
& =x^{2}\left[\frac{A_{2}(x, y) \cdot \nu(x, y)-g(x, y)}{\nu(x, y)}\right] \cdot \mu+x^{2} \cdot \tilde{B}_{2}(x, y) \cdot \bar{\mu}
\end{aligned}
$$

Considerando $P(x, y)=\frac{A_{2}(x, y) \cdot \nu(x, y)-g(x, y)}{\nu(x, y)} \quad$ e $\quad Q(x, y)=\tilde{B}_{2}(x, y)$ temos a equação:

$$
L \mu=x^{2} \cdot P(x, y) \cdot \mu+x^{2} \cdot Q(x, y) \cdot \bar{\mu},
$$

sendo $P, Q \in C^{\sigma}\left(U_{\epsilon, \delta}\right)$.

Agora, considere a função $\phi: \mathbb{R}^{2} \rightarrow \mathbb{C}$ tais que:

- $\phi \in C_{c}^{\infty}\left(\mathbb{R}^{2} ; \mathbb{C}\right)$

- $\operatorname{Supp}(\phi) \subset U_{\epsilon, \delta}$;

- $\phi \equiv 1$ num domínio compacto $\bar{U}_{\epsilon^{\prime}, \delta^{\prime}}$ de $(0,0)$, com $\bar{U}_{\epsilon^{\prime}, \delta^{\prime}} \subset U_{\epsilon, \delta}$, sendo $0<\epsilon^{\prime}<\epsilon$ e $0<\delta^{\prime}<\delta$. 
Assim, considere em $U_{\epsilon, \delta}$ a seguinte equação:

$$
L \vartheta=x^{2} \cdot P_{1}(x, y) \cdot \vartheta+x^{2} \cdot Q_{1}(x, y) \cdot \bar{\vartheta}
$$

sendo $P_{1}, Q_{1}: \mathbb{R}^{2} \rightarrow \mathbb{C}$ tais que:

$$
\begin{aligned}
& P_{1}(x, y)=\left\{\begin{array}{cl}
P(x, y) \cdot \phi(x, y) & , \quad(x, y) \in U_{\epsilon, \delta} \\
0 & , \quad(x, y) \in \mathbb{C} \backslash U_{\epsilon, \delta}
\end{array},\right. \\
& Q_{1}(x, y)=\left\{\begin{array}{cl}
Q(x, y) \cdot \phi(x, y) & , \quad(x, y) \in U_{\epsilon, \delta} \\
0 & , \quad(x, y) \in \mathbb{C} \backslash U_{\epsilon, \delta}
\end{array}\right.
\end{aligned}
$$

Note que $P_{1}, Q_{1} \in C^{\sigma}\left(\mathbb{R}^{2}\right)$.

Portanto, encontrar $\omega \in C^{1}\left(U_{\epsilon, \delta}\right)$ tal que $\omega$ seja solução da equação (3.13) é equivalente a encontrar $\mu \in C^{1}\left(U_{\epsilon, \delta}\right)$ tal que $\mu$ satisfaça a equação $(* 11)$.

Considere a função

$$
\begin{aligned}
z: U_{\epsilon, \delta} & \rightarrow z\left(U_{\epsilon, \delta}\right) \subset \mathbb{C} \\
(x, y) & \mapsto z(x, y)=x e^{i y} .
\end{aligned}
$$

Notemos que $z\left(U_{\epsilon, \delta}\right)=\left\{\zeta=\xi+i \eta \in z\left(U_{\epsilon, \delta}\right):|\zeta| \leq \epsilon,|\arg (\zeta)| \leq \delta\right.$ ou $\left.|\pi-\arg (\zeta)| \leq \delta\right\}$, $z \in C^{\infty}\left(U_{\epsilon, \delta}\right)$ e que $0 \in z\left(U_{\epsilon, \delta}\right)$.

Considere: $U_{\epsilon^{\prime}, \delta^{\prime}}^{+}=\left(0, \epsilon^{\prime}\right) \times\left(-\delta^{\prime}, \delta^{\prime}\right), U_{\epsilon^{\prime}, \delta^{\prime}}^{-}=\left(-\epsilon^{\prime}, 0\right) \times\left(-\delta^{\prime}, \delta^{\prime}\right), U_{\epsilon^{\prime}, \delta^{\prime}}^{0}=\{0\} \times\left(-\delta^{\prime}, \delta^{\prime}\right)$.

Daí, $U_{\epsilon^{\prime}, \delta^{\prime}}=U_{\epsilon^{\prime}, \delta^{\prime}}^{+} \cup U_{\epsilon^{\prime}, \delta^{\prime}}^{-} \cup U_{\epsilon^{\prime}, \delta^{\prime}}^{0}$, como podemos observar na figura 3.1.

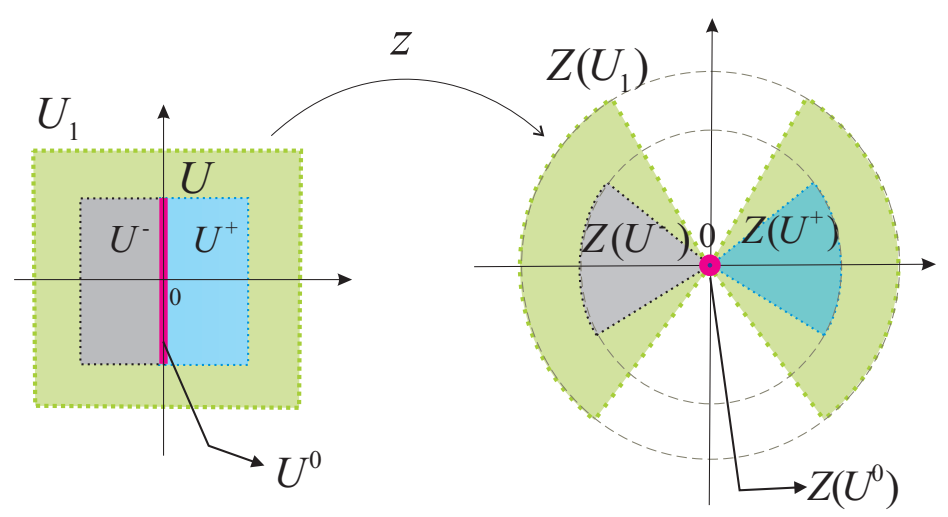

Figura 3.1: $U_{1}=U_{\epsilon, \delta}, U=U_{\epsilon^{\prime}, \delta^{\prime}}, U^{+}=U_{\epsilon^{\prime}, \delta^{\prime}}^{+}, U^{-}=U_{\epsilon^{\prime}, \delta^{\prime}}^{-}, U^{0}=U_{\epsilon^{\prime}, \delta^{\prime}}^{0}$ 
Observemos que, se $\mu \in C^{1}\left(U_{\epsilon, \delta}\right)$ é solução de $(* 11)$, para todo $(0, y) \in U_{\epsilon, \delta}^{0}$ temos

$$
\begin{aligned}
\frac{\partial \mu}{\partial y}(0, y)-i \cdot 0 \cdot \frac{\partial \mu}{\partial x}(0, y) & =L \mu(0, y) & \Rightarrow \\
\frac{\partial \mu}{\partial y}(0, y) & =0^{2} \cdot P(0, y) \cdot \mu(0, y)+0^{2} \cdot Q(0, y) \cdot \bar{\mu}(0, y) & \Rightarrow \\
\int_{0}^{y} \frac{\partial \mu}{\partial \tau}(0, \tau) d \tau & =\int_{0}^{y} 0 \cdot d \tau & \Rightarrow \\
\mu(0, y) & =\mu(0,0), &
\end{aligned}
$$

para todo $y \in(-\delta, \delta)$.

Note que $\left.z\right|_{U_{\epsilon, \delta}^{+}}$e $\left.z\right|_{U_{\epsilon, \delta}^{-}}$são difeomorfismos.

Por outro lado, para todo $(x, y) \in U_{\epsilon, \delta}^{+}, U_{\epsilon, \delta}^{-}$, temos que:

$$
\begin{aligned}
& -L(\bar{z}(x, y))=L\left(x e^{-i y}\right)=\frac{\partial}{\partial y}\left(x e^{-i y}\right)-i x \frac{\partial}{\partial x}\left(x e^{-i y}\right)=-2 i x e^{-i y}=-2 i \bar{\zeta} \\
& \text { - } L(z(x, y))=L\left(x e^{i y}\right)=\frac{\partial}{\partial y}\left(x e^{i y}\right)-i x \frac{\partial}{\partial x}\left(x e^{i y}\right)=0 .
\end{aligned}
$$

Considere $\mu^{+}=\left.\mu\right|_{U_{\epsilon, \delta}^{+}}$e $\mu^{-}=\left.\mu\right|_{U_{\epsilon, \delta}^{-}}$. Logo, o push-forward de $L \mu^{+}$pela função $\left.z\right|_{U_{\epsilon, \delta}^{+}}$e o push-forward de $L \mu^{-}$pela função $\left.z\right|_{U_{\epsilon, \delta}^{-}}$são definidos por:

$$
\left(\left.z\right|_{U_{\epsilon, \delta}^{+}}\right) *\left(L \mu^{+}\right)=\left(L \mu^{+}\right) \circ\left(\left.z\right|_{U_{\epsilon, \delta}^{+}}\right)^{-1}
$$

$\mathrm{e}$

$$
\left(\left.z\right|_{U_{\epsilon, \delta}^{-}}\right) *\left(L \mu^{-}\right)=\left(L \mu^{-}\right) \circ\left(\left.z\right|_{U_{\epsilon, \delta}^{-}}\right)^{-1}
$$

para qualquer $L \mu^{+} \in C^{\infty}\left(U_{\epsilon, \delta}^{+}\right)$e $L \mu^{-} \in C^{\infty}\left(U_{\epsilon, \delta}^{-}\right)$, respectivamente.

Assim, em $(* 11)$ temos

$$
\begin{aligned}
& \left.L \mu^{+} \circ z\right|_{U_{\epsilon, \delta}^{+}} ^{-1}=\left.\left(x^{2} P_{1} \cdot \mu^{+}\right) \circ z\right|_{U_{\epsilon, \delta}^{+}} ^{-1}+\left.\left(x^{2} Q_{1} \cdot \overline{\mu^{+}}\right) \circ z\right|_{U_{\epsilon, \delta}^{+}} ^{-1} \\
& =\left[\left.\left(x^{2} P_{1}\right) \circ z\right|_{U_{\epsilon, \delta}^{+}} ^{-1}\right]\left[\left.\mu^{+} \circ z\right|_{U_{\epsilon, \delta}^{+}} ^{-1}\right]+\left[\left.\left(x^{2} Q_{1}\right) \circ z\right|_{U_{\epsilon, \delta}^{+}} ^{-1}\right]\left[\left.\overline{\mu^{+}} \circ z\right|_{U_{\epsilon, \delta}^{+}} ^{-1}\right] \\
& =\left[\left.\left(|\zeta|^{2} P_{1}\right) \circ z\right|_{U_{\epsilon, \delta}^{+}} ^{-1}\right]\left[\left.\mu^{+} \circ z\right|_{U_{\epsilon, \delta}^{+}} ^{-1}\right]+\left[\left.\left(|\zeta|^{2} Q_{1}\right) \circ z\right|_{U_{\epsilon, \delta}^{+}} ^{-1}\right]\left[\overline{\left.\mu^{+} \circ z\right|_{U_{\epsilon, \delta}^{+}} ^{-1}}\right] .
\end{aligned}
$$


Analogamente, obtemos

$$
\left.L \mu^{-} \circ z\right|_{U_{\epsilon, \delta}^{-}} ^{-1}=\left[\left.\left(|\zeta|^{2} P_{1}\right) \circ z\right|_{U_{\epsilon, \delta}^{-}} ^{-1}\right]\left[\left.\mu^{-} \circ z\right|_{U_{\epsilon, \delta}^{-}} ^{-1}\right]+\left[\left.\left(|\zeta|^{2} Q_{1}\right) \circ z\right|_{U_{\epsilon, \delta}^{-}} ^{-1}\right]\left[\overline{\left.\mu^{-} \circ z\right|_{U_{\epsilon, \delta}^{-}} ^{-1}}\right] .
$$

Agora, considerando os pontos de $z\left(U_{\epsilon, \delta}^{+}\right)$e $z\left(U_{\epsilon, \delta}^{-}\right)$escritos na forma $z=\xi+i \eta$ temos

$$
\begin{gathered}
\xi=\frac{z+\bar{z}}{2} \text { e } \eta=\frac{z-\bar{z}}{2 i} \\
\frac{\partial}{\partial \xi}=\frac{\partial}{\partial z}+\frac{\partial}{\partial \bar{z}} \text { e } \frac{\partial}{\partial \eta}=i\left(\frac{\partial}{\partial z}-\frac{\partial}{\partial \bar{z}}\right) .
\end{gathered}
$$

Considere $\tilde{L}^{+}: C^{\infty}\left(z\left(U_{\epsilon, \delta}^{+}\right)\right) \rightarrow C^{\infty}\left(z\left(U_{\epsilon, \delta}^{+}\right)\right)$e $\tilde{L}^{-}: C^{\infty}\left(z\left(U_{\epsilon, \delta}^{-}\right)\right) \rightarrow C^{\infty}\left(z\left(U_{\epsilon, \delta}^{-}\right)\right)$ os campos vetoriais da mudança de coordenadas definidos por:

$$
\tilde{L}^{+}=L \xi(x, y) \cdot \frac{\partial}{\partial \xi}+L \eta(x, y) \cdot \frac{\partial}{\partial \eta} \quad \text { e } \quad \tilde{L}^{-}=L \xi(x, y) \cdot \frac{\partial}{\partial \xi}+L \eta(x, y) \cdot \frac{\partial}{\partial \eta},
$$

para todo $(x, y) \in U_{\epsilon, \delta}^{+} \mathrm{e}(x, y) \in U_{\epsilon, \delta}^{-}$, respectivamente.

Logo,

$$
\begin{aligned}
\tilde{L}^{ \pm} & =\left(L\left(\frac{z+\bar{z}}{2}\right)\right)(x, y) \cdot\left[\frac{\partial}{\partial z}+\frac{\partial}{\partial \bar{z}}\right]+\left(L\left(\frac{z-\bar{z}}{2 i}\right)\right)(x, y) \cdot\left[\frac{\partial}{\partial z}-\frac{\partial}{\partial \bar{z}}\right] i \\
& =\frac{1}{2}(L z+L \bar{z})(x, y) \cdot\left[\frac{\partial}{\partial z}+\frac{\partial}{\partial \bar{z}}\right]+\frac{1}{2}(L z-L \bar{z})(x, y) \cdot\left[\frac{\partial}{\partial z}-\frac{\partial}{\partial \bar{z}}\right] \\
& =L z(x, y) \cdot \frac{\partial}{\partial z}+L \bar{z}(x, y) \cdot \frac{\partial}{\partial \bar{z}}
\end{aligned}
$$

para todo $(x, y) \in U_{\epsilon, \delta}^{+}$e $(x, y) \in U_{\epsilon, \delta}^{-}$.

Daí,

$$
\tilde{L}^{+}\left(\left.\mu^{+} \circ z\right|_{U_{\epsilon, \delta}^{+}} ^{-1}\right)=L z(x, y) \cdot \frac{\partial}{\partial z}\left(\left.\mu^{+} \circ z\right|_{U_{\epsilon, \delta}^{+}} ^{-1}\right)+L \bar{z}(x, y) \cdot \frac{\partial}{\partial \bar{z}}\left(\left.\mu^{+} \circ z\right|_{U_{\epsilon, \delta}^{+}} ^{-1}\right)
$$

para todo $(x, y) \in U_{\epsilon, \delta}^{+}$.

Analogamente,

$$
\tilde{L}^{-}\left(\left.\mu^{-} \circ z\right|_{U_{\epsilon, \delta}^{-}} ^{-1}\right)=L z(x, y) \cdot \frac{\partial}{\partial z}\left(\left.\mu^{-} \circ z\right|_{U_{\epsilon, \delta}^{-}} ^{-1}\right)+L \bar{z}(x, y) \cdot \frac{\partial}{\partial \bar{z}}\left(\left.\mu^{-} \circ z\right|_{U_{\epsilon, \delta}^{-}} ^{-1}\right)
$$


para todo $(x, y) \in U_{\epsilon, \delta}^{-}$.

Por outro lado, pela proposição 1.3, temos que: $\tilde{L}^{+}\left(\left.\mu^{+} \circ z\right|_{U_{\epsilon, \delta}^{+}} ^{-1}\right)=\left.\left(L \mu^{+}\right) \circ z\right|_{U_{\epsilon, \delta}^{+}} ^{-1} \mathrm{e}$ $\tilde{L}^{-}\left(\left.\mu^{-} \circ z\right|_{U_{\epsilon, \delta}^{-}} ^{-1}\right)=\left.\left(L \mu^{-}\right) \circ z\right|_{U_{\epsilon, \delta}^{-}} ^{-1}$.

Assim, de $(* 17)$ e $(* 15)$ temos

$$
\begin{aligned}
L z(x, y) \frac{\partial}{\partial z}\left(\left.\mu^{+} \circ z\right|_{U_{\epsilon, \delta}^{+}} ^{-1}\right)+L \bar{z}(x, y) \frac{\partial}{\partial \bar{z}}\left(\left.\mu^{+} \circ z\right|_{U_{\epsilon, \delta}^{+}} ^{-1}\right)= & {\left[\left.\left(|\zeta|^{2} P_{1}\right) \circ z\right|_{U_{\epsilon, \delta}^{+}} ^{-1}\right]\left[\left.\mu^{+} \circ z\right|_{U_{\epsilon, \delta}^{+}} ^{-1}\right]+} \\
& +\left[\left.\left(|\zeta|^{2} Q_{1}\right) \circ z\right|_{U_{\epsilon, \delta}^{+}} ^{-1}\right]\left[\overline{\left.\mu^{+} \circ z\right|_{U_{\epsilon, \delta}^{+}} ^{-1}}\right],
\end{aligned}
$$

para todo $(x, y) \in U_{\epsilon, \delta}^{+}$.

De $(* 18)$ e $(* 16)$ temos

$$
\begin{aligned}
L z(x, y) \frac{\partial}{\partial z}\left(\left.\mu^{-} \circ z\right|_{U_{\epsilon, \delta}^{-}} ^{-1}\right)+L \bar{z}(x, y) \frac{\partial}{\partial \bar{z}}\left(\left.\mu^{-} \circ z\right|_{U_{\epsilon, \delta}^{-}} ^{-1}\right)= & {\left[\left.\left(|\zeta|^{2} P_{1}\right) \circ z\right|_{U_{\epsilon, \delta}^{-}} ^{-1}\right]\left[\left.\mu^{-} \circ z\right|_{U_{\epsilon, \delta}^{-}} ^{-1}\right]+} \\
& +\left[\left.\left(|\zeta|^{2} Q_{1}\right) \circ z\right|_{U_{\epsilon, \delta}^{-}} ^{-1}\right]\left[\left.\overline{\mu^{-}} \circ z\right|_{U_{\epsilon, \delta}^{-}} ^{-1}\right],
\end{aligned}
$$

para todo $(x, y) \in U_{\epsilon, \delta}^{-}$.

Considere:

$$
\begin{aligned}
& \psi^{+}=\left.\mu^{+} \circ z\right|_{U_{\epsilon, \delta}^{+}} ^{-1}: z\left(U_{\epsilon, \delta}^{+}\right) \rightarrow \mathbb{C} \text { e } \overline{\psi^{+}}=\left.\overline{\mu^{+}} \circ z\right|_{U_{\epsilon, \delta}^{+}} ^{-1}: z\left(U_{\epsilon, \delta}^{+}\right) \rightarrow \mathbb{C} ; \\
& \psi^{-}=\left.\mu^{-} \circ z\right|_{U_{\epsilon, \delta}^{-}} ^{-1}: z\left(U_{\epsilon, \delta}^{-}\right) \rightarrow \mathbb{C} \text { e } \overline{\psi^{-}}=\left.\overline{\mu^{-}} \circ z\right|_{U_{\epsilon, \delta}^{-}} ^{-1}: z\left(U_{\epsilon, \delta}^{-}\right) \rightarrow \mathbb{C} .
\end{aligned}
$$

Também, como $z \in C^{\infty}\left(U_{\epsilon, \delta}^{+}\right), z \in C^{\infty}\left(U_{\epsilon, \delta}^{-}\right), \mu^{+} \in C^{1}\left(U_{\epsilon, \delta}^{+}\right)$e $\mu^{-} \in C^{1}\left(U_{\epsilon, \delta}^{-}\right)$, temos que $\psi^{+} \in C^{1}\left(z\left(U_{\epsilon, \delta}^{+}\right)\right)$e $\psi^{-} \in C^{1}\left(z\left(U_{\epsilon, \delta}^{-}\right)\right)$.

Logo, temos

$$
\underbrace{L z(x, y)}_{=0} \cdot \frac{\partial \psi^{+}}{\partial z}+L \bar{z}(x, y) \cdot \frac{\partial \psi^{+}}{\partial \bar{z}}=|\zeta|^{2} P_{1}\left(\left.z\right|_{U_{\epsilon, \delta}^{+}} ^{-1}(\xi, \eta)\right) \cdot \psi^{+}+|\zeta|^{2} Q_{1}\left(\left.z\right|_{U_{\epsilon, \delta}^{+}} ^{-1}(\xi, \eta)\right) \cdot \overline{\psi^{+}}
$$


o que implica

$$
-2 i \bar{\zeta} \cdot \frac{\partial \psi^{+}}{\partial \bar{z}}=\zeta \cdot \bar{\zeta} \cdot P_{1}\left(\left.z\right|_{U_{\epsilon, \delta}^{+}} ^{-1}(\xi, \eta)\right) \cdot \psi^{+}+\zeta \cdot \bar{\zeta} \cdot Q_{1}\left(\left.z\right|_{U_{\epsilon, \delta}^{+}} ^{-1}(\xi, \eta)\right) \cdot \overline{\psi^{+}}
$$

$\log \mathrm{O}$

$$
\frac{\partial \psi^{+}}{\partial \bar{z}}=\frac{i}{2}\left[\zeta \cdot P_{1}\left(\left.z\right|_{U_{\epsilon, \delta}^{+}} ^{-1}(\xi, \eta)\right) \cdot \psi^{+}+\zeta \cdot Q_{1}\left(\left.z\right|_{U_{\epsilon, \delta}^{+}} ^{-1}(\xi, \eta)\right) \cdot \overline{\psi^{+}}\right]
$$

Analogamente, obtemos

$$
\frac{\partial \psi^{-}}{\partial \bar{z}}=\frac{i}{2}\left[\zeta \cdot P_{1}\left(\left.z\right|_{U_{\epsilon, \delta}^{-}} ^{-1}(\xi, \eta)\right) \cdot \psi^{-}+\zeta \cdot Q_{1}\left(\left.z\right|_{U_{\epsilon, \delta}^{-}} ^{-1}(\xi, \eta)\right) \cdot \overline{\psi^{-}}\right]
$$

Considere:

$$
\tilde{P}_{1}^{ \pm}(\xi, \eta)=\left\{\begin{array}{cll}
\left.P_{1} \circ z\right|_{U_{\epsilon, \delta}^{ \pm}} ^{-1}(\xi, \eta) & , \quad(\xi, \eta) \in z\left(U_{\epsilon, \delta}^{ \pm}\right) \\
0 & , \quad(\xi, \eta) \in \mathbb{C} \backslash z\left(U_{\epsilon, \delta}^{ \pm}\right)
\end{array}\right.
$$

$\mathrm{e}$

$$
\tilde{Q}_{1}^{ \pm}(\xi, \eta)=\left\{\begin{array}{cl}
\left.Q_{1} \circ z\right|_{U_{\epsilon, \delta}^{ \pm}} ^{-1}(\xi, \eta) & , \quad(\xi, \eta) \in z\left(U_{\epsilon, \delta}^{ \pm}\right) \\
0 & , \quad(\xi, \eta) \in \mathbb{C} \backslash z\left(U_{\epsilon, \delta}^{ \pm}\right)
\end{array} .\right.
$$

Note que $\left.P_{1} \circ z\right|_{U_{\epsilon, \delta}^{ \pm}} ^{-1}: z\left(U_{\epsilon, \delta}^{ \pm}\right) \rightarrow \mathbb{C}$ e $\left.Q_{1} \circ z\right|_{U_{\epsilon, \delta}^{ \pm}} ^{-1}: z\left(U_{\epsilon, \delta}^{ \pm}\right) \rightarrow \mathbb{C}$ são de classe $C^{\sigma}$ em $z\left(U_{\epsilon, \delta}^{ \pm}\right)$.

Assim, temos que $\tilde{P}_{1}^{ \pm} \in C^{\sigma}(\mathbb{C} \backslash\{(0,0)\})$ e $\tilde{Q}_{1}^{ \pm} \in C^{\sigma}(\mathbb{C} \backslash\{(0,0)\})$; também, $\tilde{P}_{1}^{ \pm}$e $\tilde{Q}_{1}^{ \pm}$são limitadas.

Assim, em $(* 19)$ e $(* 20)$ temos

$$
\begin{aligned}
& \frac{\partial \psi^{+}}{\partial \bar{z}}=\frac{i}{2}\left[\zeta \cdot \tilde{P}_{1}(\xi, \eta) \cdot \psi^{+}+\zeta \cdot \tilde{Q}_{1}(\xi, \eta) \cdot \overline{\psi^{+}}\right] \\
& \frac{\partial \psi^{-}}{\partial \bar{z}}=\frac{i}{2}\left[\zeta \cdot \tilde{P}_{1}(\xi, \eta) \cdot \psi^{-}+\zeta \cdot \tilde{Q}_{1}(\xi, \eta) \cdot \overline{\psi^{-}}\right] .
\end{aligned}
$$

Logo, $\zeta \cdot \tilde{P}_{1}^{ \pm}, \zeta \cdot \tilde{Q}_{1}^{ \pm} \in C^{\sigma}(\mathbb{C} \backslash\{(0,0)\})$; portanto, $\left|\zeta \cdot \tilde{P}_{1}^{ \pm}(\xi, \eta)-0\right|=|\zeta|\left|\tilde{P}_{1}^{ \pm}(\xi, \eta)\right| \leq C|\zeta|$ e $\left|\zeta \cdot \tilde{Q}_{1}^{ \pm}(\xi, \eta)-0\right|=|\zeta|\left|\tilde{Q}_{1}^{ \pm}(\xi, \eta)\right| \leq C|\zeta|$, sendo $\left|\tilde{P}_{1}^{ \pm}(\xi, \eta)\right| \leq C$ e $\left|\tilde{Q}_{1}^{ \pm}(\xi, \eta)\right| \leq C$.

Considere em $z\left(U_{\epsilon, \delta}^{ \pm}\right)$a seguintes equações

$$
\frac{\partial \psi^{+}}{\partial \bar{z}}=\frac{i}{2}\left[\tilde{P}(\xi, \eta) \cdot \psi^{+}+\tilde{Q}(\xi, \eta) \cdot \overline{\psi^{+}}\right]
$$


$\mathrm{e}$

$$
\frac{\partial \psi^{-}}{\partial \bar{z}}=\frac{i}{2}\left[\tilde{P}(\xi, \eta) \cdot \psi^{-}+\tilde{Q}(\xi, \eta) \cdot \overline{\psi^{-}}\right]
$$

sendo $\tilde{P}^{ \pm}, \tilde{Q}^{ \pm}: \mathbb{C} \rightarrow \mathbb{C}$ tais que

$$
\tilde{P}^{ \pm}(\xi, \eta)=\left\{\begin{array}{cll}
\zeta \cdot \tilde{P}_{1}^{ \pm}(\xi, \eta) & , \quad(\xi, \eta) & \in z\left(U_{\epsilon, \delta}^{ \pm}\right) \\
0 & , \quad(\xi, \eta) \in \mathbb{C} \backslash z\left(U_{\epsilon, \delta}^{ \pm}\right)
\end{array}\right.
$$

e

$$
\tilde{Q}^{ \pm}(\xi, \eta)=\left\{\begin{array}{cll}
\zeta \cdot \tilde{Q}_{1}^{ \pm}(\xi, \eta) & , \quad(\xi, \eta) \in z\left(U_{\epsilon, \delta}^{ \pm}\right) \\
0 & , \quad(\xi, \eta) \in \mathbb{C} \backslash z\left(U_{\epsilon, \delta}^{ \pm}\right)
\end{array} .\right.
$$

De $(* 21)$ e $(* 22)$ temos que $\tilde{P}^{ \pm}, \tilde{Q}^{ \pm} \in C^{\sigma}(\mathbb{C})$, com $0<\sigma<1$.

Assim, como $\psi^{+}$e $\psi^{-}$satisfazem as equações anteriores para todo $(\xi, \eta) \in z\left(U_{\epsilon^{\prime}, \delta^{\prime}}^{+}\right)$e para todo $(\xi, \eta) \in z\left(U_{\epsilon^{\prime}, \delta^{\prime}}^{-}\right)$, respectivamente, temos que $\psi^{+}$é uma função $\left[\tilde{P}^{+}, \tilde{Q}^{+}\right]$-pseudoanalítica em $z\left(U_{\epsilon^{\prime}, \delta^{\prime}}^{+}\right)$e $\psi^{-}$é uma função $\left[\tilde{P}^{-}, \tilde{Q}^{-}\right]$-pseudo-analítica em $z\left(U_{\epsilon^{\prime}, \delta^{\prime}}^{-}\right)$.

Logo, pelo teorema 2.3 (Princípio da similaridade clássico), temos que existem uma função analítica $h^{+}$no interior de $z\left(U_{\epsilon^{\prime}, \delta^{\prime}}^{+}\right)$e $s_{0}^{+} \in C^{\sigma}\left(\overline{z\left(U_{\epsilon^{\prime}, \delta^{\prime}}^{+}\right)}\right)$tais que

$$
\psi^{+}(\xi, \eta)=e^{s_{0}^{+}(\xi, \eta)} \cdot h^{+}(\xi, \eta)
$$

para todo $(\xi, \eta) \in z\left(U_{\epsilon^{\prime}, \delta^{\prime}}^{+}\right)$. Portanto, $\psi^{+} \in C^{\sigma}\left(z\left(U_{\epsilon^{\prime}, \delta^{\prime}}^{+}\right)\right)$.

Analogamente, pelo teorema 2.3 (Princípio da similaridade clássico), temos que existem uma função analítica $h^{-}$no interior de $z\left(U_{\epsilon^{\prime}, \delta^{\prime}}^{-}\right)$e $s_{0}^{-} \in C^{\sigma}\left(\overline{z\left(U_{\epsilon^{\prime}, \delta^{\prime}}^{-}\right)}\right)$tais que

$$
\psi^{-}(\xi, \eta)=e^{s_{0}^{-}(\xi, \eta)} \cdot h^{-}(\xi, \eta)
$$

para todo $(\xi, \eta) \in z\left(U_{\epsilon^{\prime}, \delta^{\prime}}^{-}\right)$. Portanto, $\psi^{-} \in C^{\sigma}\left(z\left(U_{\epsilon^{\prime}, \delta^{\prime}}^{-}\right)\right)$.

Observemos que:

$$
\begin{aligned}
& \psi^{+}(\xi, \eta)=\psi^{+}\left(\left.z\right|_{U_{\epsilon^{\prime}, \delta^{\prime}}^{+}}(x, y)\right)=\mu^{+}(x, y), \\
& \psi^{-}(\xi, \eta)=\psi^{-}\left(\left.z\right|_{U_{\epsilon^{\prime}, \delta^{\prime}}^{-}}(x, y)\right)=\mu^{-}(x, y),
\end{aligned}
$$

para todo $(x, y) \in U_{\epsilon^{\prime}, \delta^{\prime}}^{+}$e $(x, y) \in U_{\epsilon^{\prime}, \delta^{\prime}}^{-}$, respectivamente. 
Logo, por $(* 12)$ temos

$$
\lim _{\zeta \rightarrow 0} \psi^{ \pm}(\zeta)=\lim _{\substack{x \rightarrow 0 \\ y \rightarrow y_{0}}} \mu^{ \pm}(x, y)=\mu^{ \pm}\left(0, y_{0}\right)=\mu(0,0)
$$

Por outro lado, de $(* 23)$ temos que

$$
\begin{aligned}
\psi^{+}(\xi, \eta) & =e^{s_{0}^{+}(\xi, \eta)} \cdot h^{+}(\xi, \eta)=e^{s_{0}^{+}(\xi, \eta)-s_{0}^{+}(0,0)} \cdot\left[e^{s_{0}^{+}(0,0)} \cdot h^{+}(\xi, \eta)\right] \\
& =e^{\tilde{s}^{+}(\xi, \eta)} \cdot H^{+}(\xi, \eta)
\end{aligned}
$$

sendo $\tilde{s}^{+}(\xi, \eta)=s_{0}^{+}(\xi, \eta)-s_{0}^{+}(0,0)$ e $H^{+}(\xi, \eta)=e^{s_{0}^{+}(0,0)} \cdot h^{+}(\xi, \eta)$, para todo $(\xi, \eta) \in z\left(U_{\epsilon^{\prime}, \delta^{\prime}}^{+}\right)$. Note que, $\tilde{s}^{+}(0,0)=(0,0)$.

Analogamente,

$$
\begin{aligned}
\psi^{-}(\xi, \eta) & =e^{s_{0}^{-}(\xi, \eta)} \cdot h^{-}(\xi, \eta)=e^{s_{0}^{-}(\xi, \eta)-s_{0}^{-}(0,0)} \cdot\left[e^{s_{0}^{-}(0,0)} \cdot h^{-}(\xi, \eta)\right] \\
& =e^{\tilde{s}^{-}(\xi, \eta)} \cdot H^{-}(\xi, \eta)
\end{aligned}
$$

sendo $\tilde{s}^{-}(\xi, \eta)=s_{0}^{-}(\xi, \eta)-s_{0}^{-}(0,0)$ e $H^{-}(\xi, \eta)=e^{s_{0}^{-}(0,0)} \cdot h^{-}(\xi, \eta)$, para todo $(\xi, \eta) \in z\left(U_{\epsilon^{\prime}, \delta^{\prime}}^{-}\right)$. Note que $\tilde{s}^{-}(0,0)=(0,0)$.

Definamos $\psi: z\left(U_{\epsilon^{\prime}, \delta^{\prime}}\right) \rightarrow \mathbb{C}$, sendo

$$
\psi(\xi, \eta)=\left\{\begin{array}{cll}
\psi^{+}(\xi, \eta) & , \quad(\xi, \eta) \in z\left(U_{\epsilon^{\prime}, \delta^{\prime}}^{+}\right) \\
\mu(0,0) & , \quad(\xi, \eta)=(0,0) \\
\psi^{-}(\xi, \eta) & , \quad(\xi, \eta) \in z\left(U_{\epsilon^{\prime}, \delta^{\prime}}^{-}\right)
\end{array} .\right.
$$

Por construção, $\psi$ é função $[\tilde{P}, \tilde{Q}]$-pseudo-analítica em $z\left(U_{\epsilon^{\prime}, \delta^{\prime}}\right)$.

Também, de $(* 25)$ temos

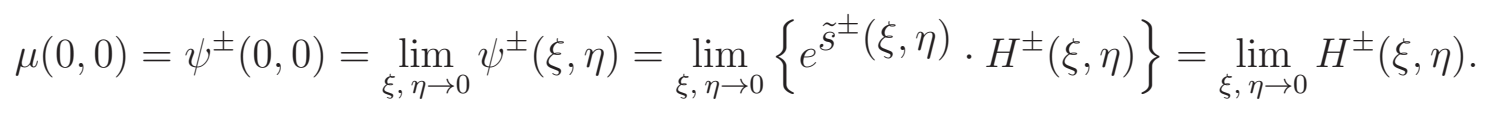

Podemos definir $H^{+}(0,0)=H^{-}(0,0)=\mu(0,0)$. 
Agora, definamos as funções $\tilde{s}: z\left(U_{\epsilon^{\prime}, \delta^{\prime}}\right) \rightarrow \mathbb{C}$ e $H: z\left(U_{\epsilon^{\prime}, \delta^{\prime}}\right) \rightarrow \mathbb{C}$, por

$$
\tilde{s}(\xi, \eta)=\left\{\begin{array}{cl}
\tilde{s}^{+}(\xi, \eta) \quad, \quad(\xi, \eta) \in z\left(U_{\epsilon^{\prime}, \delta^{\prime}}^{+}\right) \\
(0,0) \quad, \quad(\xi, \eta)=(0,0) \\
\tilde{s}^{-}(\xi, \eta), & (\xi, \eta) \in z\left(U_{\epsilon^{\prime}, \delta^{\prime}}^{-}\right)
\end{array}\right.
$$

$\mathrm{e}$

$$
H(\xi, \eta)=\left\{\begin{array}{cl}
H^{+}(\xi, \eta) \quad, \quad(\xi, \eta) \in z\left(U_{\epsilon^{\prime}, \delta^{\prime}}^{+}\right) \\
\mu(0,0), \quad(\xi, \eta)=(0,0) \\
H^{-}(\xi, \eta), & (\xi, \eta) \in z\left(U_{\epsilon^{\prime}, \delta^{\prime}}^{-}\right)
\end{array} .\right.
$$

Temos:

- $\tilde{s} \in C^{0}\left(z\left(\overline{U_{\epsilon^{\prime}, \delta^{\prime}}}\right)\right)$ e $\tilde{s} \in C^{\sigma}\left(z\left(\overline{U_{\epsilon^{\prime}, \delta^{\prime}}}\right) \backslash\{(0,0)\}\right)$;

- $H \in C^{0}\left(z\left(U_{\epsilon^{\prime}, \delta^{\prime}}\right)\right.$ e $H$ é holomorfa no $\operatorname{int}\left(z\left(U_{\epsilon^{\prime}, \delta^{\prime}}\right)\right)$.

Além disso, para $(\xi, \eta) \in z\left(\overline{U_{\epsilon^{\prime}, \delta^{\prime}}^{+}}\right)$temos

$$
\left|\tilde{s}^{+}(\xi, \eta)-\tilde{s}^{+}(0,0)\right|=\left|\tilde{s}_{0}^{+}(\xi, \eta)-\tilde{s}_{0}^{+}(0,0)-0\right| \leq C|(\xi, \eta)-(0,0)|^{\sigma} .
$$

Também, para $(\xi, \eta) \in z\left(\overline{\left(\overline{\epsilon^{\prime}, \delta^{\prime}}\right.}\right)$ temos

$$
\left|\tilde{s}^{-}(\xi, \eta)-\tilde{s}^{-}(0,0)\right|=\left|\tilde{s}_{0}^{-}(\xi, \eta)-\tilde{s}_{0}^{-}(0,0)-0\right| \leq C|(\xi, \eta)-(0,0)|^{\sigma} .
$$

Logo, $\tilde{s} \in C^{\sigma}\left(z\left(\overline{U_{\epsilon^{\prime}, \delta^{\prime}}}\right)\right)$.

Finalmente, note que $\psi: z\left(U_{\epsilon^{\prime}, \delta^{\prime}}\right) \rightarrow \mathbb{C}$ pode ser escrita como

$$
\psi(\xi, \eta)=e^{\tilde{s}(\xi, \eta)} \cdot H(\xi, \eta)
$$

para todo $(\xi, \eta) \in z\left(U_{\epsilon^{\prime}, \delta^{\prime}}\right)$.

Afirmação: $\psi$ é Hölder contínua em $z\left(\overline{U_{\epsilon^{\prime}, \delta^{\prime}}}\right)$. 
De fato, seja $(\xi, \eta),(\xi, 0),(0,0) \in z\left(\overline{U_{\epsilon^{\prime}, \delta^{\prime}}}\right)$, temos que

$$
\begin{aligned}
|\psi(\xi, 0)-\psi(0,0)| & =|\mu(x, 0)-\mu(0,0)| \\
& \leq\left|\frac{\partial \mu}{\partial x}(\bar{x}, 0)\right| \cdot|x| \quad \quad \text { (por T.V.M) } \\
& \leq C_{1}|x| \leq C_{1}|x|^{1-\sigma} \cdot|x|^{\sigma} \leq C_{1} \cdot\left(\epsilon^{\prime}\right)^{1-\sigma} \cdot|x|^{\sigma} \\
& \leq C|x|^{\sigma}=C|(\xi, 0)|^{\sigma},
\end{aligned}
$$

sendo que $\left|\frac{\partial \mu}{\partial x}(\bar{x}, 0)\right| \leq C_{1}$ e $C=C_{1} \cdot\left(\epsilon^{\prime}\right)^{1-\sigma}>0$.

Daí,

$$
\begin{aligned}
|\psi(\xi, \eta)-\psi(0,0)| & \leq|\psi(\xi, \eta)-\psi(\xi, 0)|+|\psi(\xi, 0)-\psi(0,0)| \\
& \leq C|(\xi, \eta)-(\xi, 0)|^{\sigma}+C|(\xi, 0)|^{\sigma} \\
& \leq C(|(\xi, \eta)|+|(\xi, 0)|)^{\sigma}+C|(\xi, 0)|^{\sigma} \\
& \leq C(2|(\xi, \eta)|)^{\sigma}+C|(\xi, \eta)|^{\sigma}, \quad(\text { pois }|(\xi, 0)| \leq|(\xi, \eta)|) \\
& \leq C\left(2^{\sigma}+1\right)|(\xi, \eta)|^{\sigma} \leq C\left(2^{\sigma}+1\right)|(\xi, \eta)-(0,0)|^{\sigma} \\
& \leq K_{1}|(\xi, \eta)-(0,0)|^{\sigma},
\end{aligned}
$$

sendo $K_{1}=C\left(2^{\sigma}+1\right)>0$. Portanto, $\psi$ é Hölder contínua em $z\left(\overline{U_{\epsilon^{\prime}, \delta^{\prime}}}\right)$.

Por outro lado, de $(* 26)$ temos

$$
H(\xi, \eta)=\psi(\xi, \eta) \cdot e^{-\tilde{s}(\xi, \eta)}
$$

Logo, $H \in C^{\sigma}\left(z\left(\overline{U_{\epsilon^{\prime}, \delta^{\prime}}}\right)\right)$, pois $\psi \in C^{\sigma}\left(z\left(\overline{U_{\epsilon^{\prime}, \delta^{\prime}}}\right)\right)$ e $e^{-\tilde{s}} \in C^{\sigma}\left(\overline{z\left(U_{\epsilon^{\prime}, \delta^{\prime}}\right)}\right)$.

Finalmente, compondo novamente $(* 26)$, temos $\psi \circ z=e^{(\tilde{s} \circ z)} \cdot(H \circ z)$.

Assim, dado $(x, y) \in U_{\epsilon^{\prime}, \delta^{\prime}}$, temos

$$
(\psi \circ z)(x, y)=e^{(\tilde{s} \circ z)(x, y)} \cdot(H \circ z)(x, y)
$$


isto é,

$$
\mu(x, y)=e^{(\tilde{s} \circ z)(x, y)} \cdot H(z(x, y))
$$

Logo, temos que $(* 27)$ é solução da equação $(* 11)$. Mas, sabemos que $\mu=\frac{\omega}{\nu}, \operatorname{com} \nu \neq 0$. Assim, de $(* 27)$, temos

$$
\begin{array}{lll}
\frac{\omega(x, y)}{\nu(x, y)}=e^{(\tilde{s} \circ z)(x, y)} \cdot H(z(x, y)) & \Rightarrow \\
\omega(x, y) & =\nu(x, y) \cdot e^{(\tilde{s} \circ z)(x, y)} \cdot H(z(x, y)) & \Rightarrow \\
\omega(x, y) & =e^{(\tilde{s} \circ z)(x, y)+\log \nu(x, y)} \cdot H(z(x, y)), &
\end{array}
$$

para todo $(x, y) \in U_{\epsilon^{\prime}, \delta^{\prime}}$.

Portanto, de $(* 28)$ temos que $\omega$ tem a forma procurada.

Reciprocamente, com a mesma notação que acima, seja $H$ uma função holomorfa no interior de $z\left(U_{\epsilon^{\prime}, \delta^{\prime}}\right)$ e $H \in C^{\sigma}\left(\operatorname{int}\left(z\left(U_{\epsilon^{\prime}, \delta^{\prime}}\right)\right)\right)$. Logo, pelo teorema 2.4 (Princípio da similaridade clássico), temos que existe uma função $\tilde{s} \in C^{\sigma}\left(\overline{z\left(U_{\epsilon^{\prime}, \delta^{\prime}}\right)}\right)$, tal que $\psi(\xi, \eta)=e^{\tilde{s}}(\xi, \eta) \cdot H(\xi, \eta)$ é $[\tilde{P}, \tilde{Q}]$-pseudo-analítica em $z\left(U_{\epsilon^{\prime}, \delta^{\prime}}\right)$. Logo, como $\psi=\mu \circ z^{-1}$, segue que a função dada por $(* 28)$ satisfaz $(3.14)$, para todo $(x, y) \in U_{\epsilon^{\prime}, \delta^{\prime}}$. 



\section{Referências Bibliográficas}

[1] Ahlfors, L. V. Lectures on quasiconformal mappings. D. Van Nostrand Company. Inc. Princeton, New Jersey (1966).

[2] Begehr, H. G. W. Complex analytic methods for partial differential equations: an introductory text. World Scientific Publishing Co. Pte. Ltd, Singapore (1994).

[3] Bell, S. R. The Cauchy transform, potential theory, and conformal mapping. N.Y., Boca Raton, Florida: CRC Press, Inc (1992).

[4] Conway, J. B. Functions of one complex variable. Springer-Verlag, New York (1978).

[5] Courant, R. e Hilbert L. Methods of mathematical physics, Vol. I. Wiley classics Edition published. New York: Interscience (1989).

[6] Courant, R. e Hilbert L. Methods of mathematical physics, Vol. II. Wiley classics library, Wiley-VCH Verlag GmbH \& Co. KGaA (1962).

[7] Fernandez, C. e Bernardes, N. Introdução às Funções de uma Variável Complexa. Sociedade Brasileira de Matemática. Brasil (2006).

[8] Folland, G. B. Introduction to partial differential equations. Princeton University Press and University of Tokio Press. Princeton, New Jersey (1976).

[9] Hounie, J. Teoria elementar das distribuições. $12^{\circ}$ Colóquio brasileiro de matemática, Poços de Caldas (1979).

[10] Lawrence, C. E. Partial Differential Equations. American Mathematical Society, Providence (1998).

[11] Lima, E. L. Análise Real, Vol. 1. Rio de Janeiro, IMPA (2008). 
[12] Lima, E. L. Curso de Análise, Vol. 2. Projeto Euclides. Rio de Janeiro, IMPA (2008).

[13] Lima, E. L. Espaços métricos. Projeto Euclides. Rio de Janeiro, IMPA (2009).

[14] Lima, E. L. Variedades diferenciáveis. Rio de Janeiro, IMPA (2010).

[15] Meziani, A. On the similarity principle for planar vector fields: application to second order PDE, J. Differential Equations, (1)157 (1999), 1-19.

[16] Vekua, I. V. Generalized Analytic Functions. Pergamon, Oxford (1962). 